\title{
TRANSPORTE IÓNICO EN ELECTROLITOS SÓLIDOS MICROESTRUCTURADOS
}

Mag. Ing. Ignacio J. Curto Sillamoni

Tesis presentada ante la Facultad de Ingeniería de la Universidad Nacional de La Plata para acceder al grado académico de

DOCTOR EN INGENIERÍA

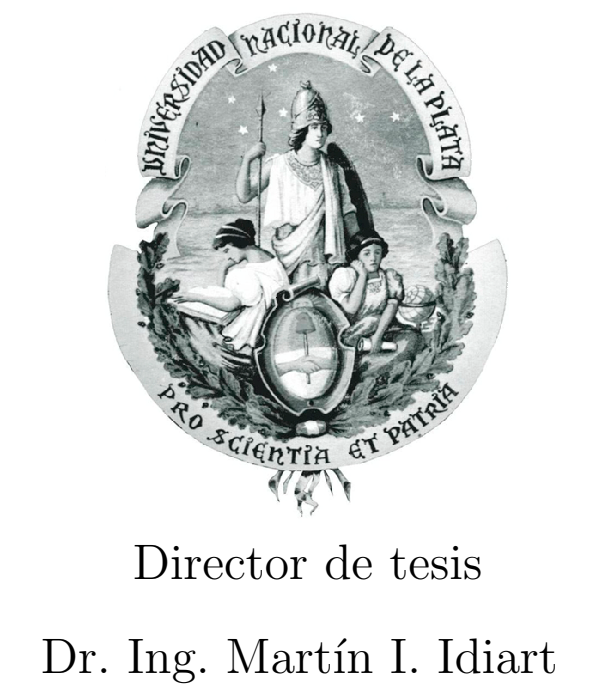

Departamento de Aeronáutica - Facultad de Ingeniería

Universidad Nacional de La Plata

La Plata, Argentina

Marzo de 2017 



\section{Prólogo}

La presente tesis doctoral se entrega ante la Facultad de Ingeniería de la Universidad Nacional de La Plata como requisito para la obtención del grado de Doctor en Ingeniería. El trabajo en ella descripto se desarrolló en el Departamento de Aeronáutica entre los años 2011 y 2016, con el apoyo del Grupo de Ensayos Mecánicos Aplicados, del Laboratorio de Polímeros - del Instituto de Fisicoquímica Teórica y Aplicada- y del Laboratorio de Investigaciones de Metalurgia Física, todos ellos dependientes de la mencionada Universidad. Dichos trabajos fueron parcialmente financiados por

- una Beca Doctoral otorgada a través del "Programa de Fromación de Doctores en Áreas Tecnológicas Prioritarias - Programa Recursos Humanos (PRH)" co-financiado por la Agencia Nacional de Promoción Científica y Tecnológica y la Universidad Nacional de La Plata (PRH-2007-15);

- una Beca Doctoral tipo II otorgada por el CONICET;

- un Subsidio para Jóvenes Investigadores otorgado por la Universidad Nacional de La Plata;

- un Proyecto de Investigación y Desarrollo acreditado por la Universidad Nacional de La Plata (I-2013-179).

Parte de los resultados obtenidos fueron presentados en

- I. J. Curto Sillamoni, M. I. Idiart (2016) Nonlinear ionic transport through microstructured solid electrolytes: homogenization estimates. Modelling and Simulation in 
Materials Science and Engineering. Modelling and Simulation in Materials Science and Engineering 24, 7.

- I. J. Curto Sillamoni, M. I. Idiart (2015) A model problem concerning ionic transport in microstructured solid electrolytes. Continuum Mechanics and Thermodynamics 27, 941-957.

- I. J. Curto Sillamoni, M. I. Idiart (2015) Transporte iónico en electrolitos sólidos microestructurados. Ponencia en el $3^{\circ}$ Seminario ECAMAT, Instituto de Estudios de Ciencia y Tecnología, Academia Nacional de Ciencias de Buenos Aires.

- I. J. Curto Sillamoni, M. I. Idiart (2014) A model problem concerning the ionic transport in microstructured solid electrolytes. Ponencia en el XXI Congreso sobre Métodos Numéricos y sus Aplicaciones, Bariloche, Argentina.

- I. J. Curto Sillamoni, M. I. Idiart (2014) A model problem concerning the ionic transport in microstructured solid electrolytes. Ponencia en el $3^{\circ}$ Congreso Argentino de Ingeniería Aeronáutica, La Plata, Argentina.

- I. J. Curto Sillamoni, M. I. Idiart (2012) Homogenization estimates for the nonlinear transport properties of isotropic particulate composites. Ponencia en el X Congreso Argentino de Mecánica Computacional, Salta, Argentina. 


\section{Índice general}

\section{Prólogo}

Resumen

2. Caso de Estudio $\quad 5$

2.1. Óxidos de polietileno dopados ........................ 5

2.2. Estudio experimental . . . . . . . . . . . . . . . . . . . . 9

2.3. Obtención de las películas poliméricas . . . . . . . . . . . . . . . 11

2.4. Medición de conductividad iónica . . . . . . . . . . . . . . . 13

2.5. Medición de la cristalinidad . . . . . . . . . . . . . . . . . . . 17

2.5.1. Calorimetría Diferencial de Barrido . . . . . . . . . . . . . . 17

2.5.2. Preparación de muestras de DSC . . . . . . . . . . . . . . . 19

2.5.3. Difracción de Rayos X-XRD . . . . . . . . . . . . . . . . . . 19

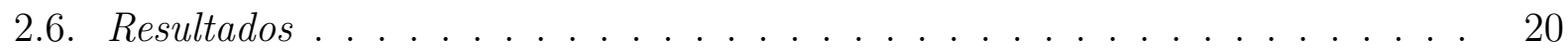

3. Marco Teórico 25

4. Respuesta Efectiva $\quad 35$

4.1. Transporte iónico lineal . . . . . . . . . . . . . . . . . . . 35

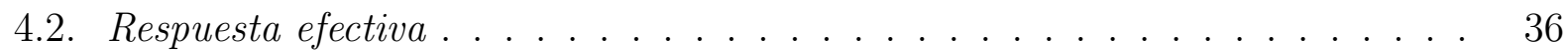

4.2.1. Problema con múltiples escalas . . . . . . . . . . . . . . . 36

4.2.2. Limites . . . . . . . . . . . . . . . . . . . . . . . . . . . 38 
4.2.3. Problema Homogenizado . . . . . . . . . . . . . . . . . . . . . 41

$\begin{array}{ll}\text { 5. Problema Modelo Lineal } & 47\end{array}$

5.1. Problema modelo de transporte iónico lineal . . . . . . . . . . . . . . . . . 47

5.2. El efecto de la cristalinidad y la concentración de sales . . . . . . . . . . . 53

5.3. El efecto de inclusiones aislantes . . . . . . . . . . . . . . . . . . . 58

5.4. Observaciones finales . . . . . . . . . . . . . . . . . . . 62

6. Transporte Iónico No Lineal $\quad 65$

6.1. Respuesta Efectiva ........................ 65

6.2. Estimaciones de Homogenización Lineal de Comparación . . . . . . . . . . . 67

6.2.1. Método de Homogenización Lineal de Comparación . . . . . . . . . . 67

6.2.2. Compuestos con fases constitutivas isótropas . . . . . . . . . . . . 69

6.2.3. Estimaciones duales . . . . . . . . . . . . . . . . . . 70

$\begin{array}{ll}\text { 7. Problemas Modelos No Lineales } & 73\end{array}$

7.1. Materiales de dos fases con disipación tipo ley de la potencia . . . . . . . . . 73

7.2. Resultados para sistemas de tipo matriz-inclusión . . . . . . . . . . . 75

7.3. Resultados para sistemas de tipo granulares . . . . . . . . . . . . . . . 80

$\begin{array}{lr}\text { 8. Conclusiones } & 87\end{array}$

$\begin{array}{lr}\text { Apéndice } & 90\end{array}$

$\begin{array}{ll}\text { Referencias } & 91\end{array}$ 


\section{Índice de figuras}

2.1. Esquema de la variación de la microestructura del PEO con la temperatura y la concentración de $\mathrm{LiClO}_{4}$ (extraído de Fullerton-Shirey \& Maranas 2009). . . . . . . . . . . .

2.2. Microestructuras de polímeros semi-cristalinos a temperatura ambiente: a) estructuras esferulíticas de un PEO dopado con perclorato de litio (extraído de Marzantowicz 2008), b) estructura granular en un polietileno cristalizado (extraído de Boudet 2003). . . . . . . .

2.3. Conductividad iónica del PEO como función de la temperatura: a) PEO con diversas sales de litio, b) PEO con diversos plastificadores y partículas cerámicas. Extraído de Fergus (2010). 8

2.4. Variación de la conductividad iónica longitudinal a temperatura ambiente con la deformación en PEO dopado con $\mathrm{LiClO}_{4}$. PEO-nn corresponde a un PEO con $n n$ mol \% de sales. Extraído de Minami et al. (2005). . . . . . . . . . . . . . . . . . . . . . . .

2.5. Microestructura de LiI-PEO no deformada (izquierda) y deformada (derecha). Extraído de Golodnitsky \& Peled (2000). . . . . . . . . . . . . . . . . . . . . . . . .

2.6. a) Imágenes de las películas casteadas, cada una mide 2.7 x $1.7 \mathrm{~cm}$ aproximadamente. b) Molde de teflon. En la parte superior se pueden observar los hilos de titanio asegurados en el molde y las primeras 6 divisiones contando desde la izquierda están llenas con PEO. El

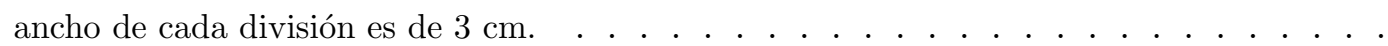

2.7. a) Esquema del método de las cuatro puntas. b) Dispositivo de teflon para ensayo de conductividad de cuatro puntas. . . . . . . . . . . . . . . . . . . . . . . . . .

2.8. Cápsulas de aluminio utilizadas para contener la muestra. De izquierda a derecha: cápsula vacía, cápsula con película de PEO dentro, tapa de las cápsulas, cápsula con PEO, cerrada con su tapa y sellada lista para ser introducida en el equipo de medición. El tamaño de cada división cuadriculada es de 5 x $5 \mathrm{~mm}$. . . . . . . . . . . . . . . . . . . . . . . 
2.9. Mediciones de conductividad vs. tiempo a) películas de concentración PEO:Li=10:1 b) películas de concentración PEO:Li=20:1. . . . . . . . . . . . . . . .

2.10. Mediciones variación porcentual de la conductividad vs. tiempo a) películas de concentración PEO:Li=10:1 b) películas de concentración PEO:Li=20:1 . . . . . . . . . . . .

2.11. Cristalinidad vs. tiempo, películas de concentración PEO:Li=20:1 (probetas 2 y 3). a) Critalinidad porcentual. b) Aumento en relación al día 1. . . . . . . . . . . . . . . .

2.12. Fracción de cambio de la conductividad y la cristalinidad, respecto al valor inicial vs. tiempo para películas de concentración PEO:Li=20:1. a) Probeta 20:1-2 b) Probeta 20:1-3. Obtenidas por DSC y por XRD. . . . . . . . . . . . . . . . .

5.1. Esquema del ensamble de cilindros de Schulgasser utilizado en los modelos con y sin inclusiones. 48

5.2. (a)-(c) Movilidad efectiva de las especies $\alpha$ en un electrolito polimérico semi-cristalino sin anillos amorfos $\left(f_{r}=0\right)$, normalizada con la correspondiente movilidad $m_{\alpha}^{(a)}$ de la fase amorfa, versus cristalinidad $f$, para el caso de $m_{\alpha}^{\|}>m_{\alpha}^{\perp}$; gráficas para: (a) $c_{0 \alpha}^{(c r)} / c_{0 \alpha}^{(a)}=$ 10, $m_{\alpha}^{\|} / m_{\alpha}^{\perp}=1000$, y diferentes contrastes en las movilidades $\left(m_{\alpha}^{\|}+m_{\alpha}^{\perp}\right) /\left(2 m_{\alpha}^{(a)}\right) ;(\mathrm{b})$ $c_{0 \alpha}^{(c r)} / c_{0 \alpha}^{(a)}=10,\left(m_{\alpha}^{\|}+m_{\alpha}^{\perp}\right) /\left(2 m_{\alpha}^{(a)}\right)=5$, y diferentes proporciones de anisotropía en la movilidad $m_{\alpha}^{\|} / m_{\alpha}^{\perp}$; (c) $\left(m_{\alpha}^{\|}+m_{\alpha}^{\perp}\right) /\left(2 m_{\alpha}^{(a)}\right)=5, m_{\alpha}^{\|} / m_{\alpha}^{\perp}=1000$, y varios contrastes en las concentraciones de referencia $c_{0 \alpha}^{(c r)} / c_{0 \alpha}^{(a)}$. (d) Conductividad efectiva de una clase de electrolitos con microestructura variable, normalizada con una conductividad de referencia $\kappa_{0}$, en función de la relación de concentración de sales de litio por cada mol de PEO, para diferentes cristalinidades de las cilindritas $f_{c}^{c r} \ldots \ldots \ldots \ldots \ldots$ 
5.3. (a)-(c) Movilidad efectiva de las especies $\alpha$ en un electrolito polimérico semi-cristalino sin anillos amorfos $\left(f_{r}=0\right)$, normalizada con la correspondiente movilidad $m_{\alpha}^{(a)}$ de la fase amorfa, versus cristalinidad $f$, para el caso de $m_{\alpha}^{\perp}>m_{\alpha}^{\|}$; gráficas para: (a) $c_{0 \alpha}^{(c r)} / c_{0 \alpha}^{(a)}=$ $2, m_{\alpha}^{\|} / m_{\alpha}^{\perp}=1000, \mathrm{y}$ diferentes contrastes en las movilidades $\left(m_{\alpha}^{\|}+m_{\alpha}^{\perp}\right) /\left(2 m_{\alpha}^{(a)}\right) ;(\mathrm{b})$ $c_{0 \alpha}^{(c r)} / c_{0 \alpha}^{(a)}=2,\left(m_{\alpha}^{\|}+m_{\alpha}^{\perp}\right) /\left(2 m_{\alpha}^{(a)}\right)=5$, y diferentes proporciones de anisotropía en la movilidad $m_{\alpha}^{\|} / m_{\alpha}^{\perp}$; (c) $\left(m_{\alpha}^{\|}+m_{\alpha}^{\perp}\right) /\left(2 m_{\alpha}^{(a)}\right)=5, m_{\alpha}^{\|} / m_{\alpha}^{\perp}=1000$, y varios contrastes en las concentraciones de referencia $c_{0 \alpha}^{(c r)} / c_{0 \alpha}^{(a)}$. (d) Conductividad efectiva de una clase de electrolitos con microestructura variable, normalizada con una conductividad de referencia $\kappa_{0}$, en función de la relación de concentración de sales de litio por cada mol de PEO, para diferentes cristalinidades de las cilindritas $f_{c}^{c r} \ldots \ldots \ldots \ldots \ldots$

5.4. Propiedades de transporte de un electrolito compuesto (EO:Li=10) como función del contenido de partículas, para diferentes tasas de crecimiento de las interfases y diferentes relaciones de concentración $c_{0-}^{(i n t)} / c_{0+}^{(i n t)}$ : (a) conductividad efectiva, adimensionalizada con la conductividad de referencia $\kappa_{0} ;$ (b) número de transporte catiónico efectivo. . . . . . . . .

7.1. Estimaciones del tipo MGA para la resistividad efectiva de un compuesto de dos fases con no linealidad material $(m)$, concentración de inclusiones $\left(\theta^{(2)}\right)$ y relación de resistividades $\left(G_{0}^{(2)} / G_{0}^{(1)}\right) \ldots \ldots \ldots \ldots \ldots$

7.2. Estimaciones del método EMA para la resistividad efectiva de un compuesto bifásico en función de la no linealidad material $(m)$ y de la concentración de inclusiones $\left(\theta^{(2)}\right) .81$

A1. Curva resultado del análisis de calorimetría diferencial para probeta de PEO sin

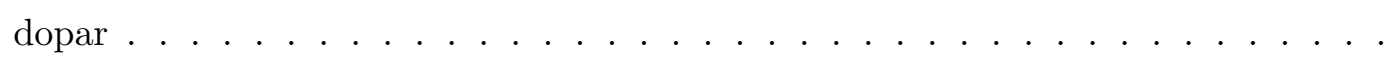

A2. Curva resultado del análisis de calorimetría diferencial de la Probeta 2 de PEO:Li $20: 1$ - Día $1 \ldots \ldots \ldots \ldots \ldots \ldots \ldots$

A3. Curva resultado del análisis de calorimetría diferencial de la Probeta 2 de PEO:Li $20: 1$ - Día $17 \ldots \ldots \ldots \ldots \ldots \ldots \ldots \ldots \ldots \ldots \ldots \ldots$

A4. Curva resultado del análisis de calorimetría diferencial de la Probeta 2 de PEO:Li

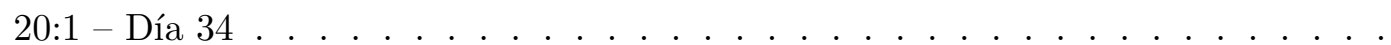

A5. Curva resultado del análisis de calorimetría diferencial de la Probeta 2 de PEO:Li $20: 1$ - Día $65 \ldots \ldots \ldots \ldots \ldots \ldots$ 
A6. Curva resultado del análisis de calorimetría diferencial de la Probeta 3 de PEO:Li 20:1 - Día $17 \ldots \ldots \ldots$. . . . . . . . . . . . . . . . . 97

A7. Curva resultado del análisis de calorimetría diferencial de la Probeta 3 de PEO:Li 20:1 - Día $34 \ldots \ldots \ldots$. . . . . . . . . . . . . . . . . . . . . . . . . . 98

A8. Curva resultado del análisis de calorimetría diferencial de la Probeta 3 de PEO:Li 20:1 - Día 65 . . . . . . . . . . . . . . . . . . . . . . . . . . . . 99

A9. Curva resultado de los análisis de difracción de rayos X de probeta de PEO:Li 20:1

- Días 17 y $45 \ldots \ldots \ldots \ldots$. . . . . . . . . . . . . . . 100 


\section{Índice de tablas}

2.1. Nomenclatura y espesor promedio de las probetas casteadas. . . . . . . . . . . . . . 12

2.2. Rangos utilizados en los análisis de DSC . . . . . . . . . . . . . . . . . . 18 


\section{Resumen}

Se aborda el estudio de las propiedades de transporte iónico en electrolitos sólidos microestructurados. Inicialmente se presentan experimentos que permiten observar de manera preliminar y cualitativa el efecto de los cambios de la cristalinidad y concentración de litio sobre la conductividad iónica en polímeros semicristalinos.

En el aspecto teórico, el trabajo de correlacionar las propiedades de transporte macroscópicas con la microestructura subyacente en electrolitos sólidos es una tarea compleja que generalmente requiere resolver un sistema de ecuaciones de electrodifusión no lineales, acopladas, con coeficientes variables. Se considera el transporte de múltiples especies iónicas por difusión y migración a través de electrolitos sólidos microestructurados en presencia de fuertes campos eléctricos. Se asumen relaciones constitutivas derivadas de potenciales de disipación y energía convexos que garantizan la consistencia termodinámica del sistema. La respuesta efectiva es determinada a partir de un análisis de convergencia multi-escala de las ecuaciones de campo correspondientes. La respuesta efectiva homogenizada involucra varios tensores efectivos pero todos ellos requieren la solución de sólo un problema de conductividad estándar sobre el elemento de volumen representativo. Se deriva, a modo de ejemplo, un modelo multiescala de un electrolito polimérico semicristalino con morfología esferulítica, aplicando la teoría desarrollada a una clase específica de microgeometrías bidimensionales para las cuales la respuesta efectiva puede ser calculada de manera exacta. Se deriva también un modelo más general en el que se considera una dispersión aleatoria de partículas y sus interfaces con el medio. En ambos casos se obtienen expresiones explícitas para los parámetros materiales efectivos. Dichos modelos son utilizados para explorar el efecto de la cristalinidad y el contenido de partículas sobre la respuesta efectiva. Las predicciones son consistentes con 
resultados experimentales recientes en sistemas de óxido de polietileno (PEO) dopado que sugieren que la fase cristalina y anisótropa, a lo largo de ciertas direcciones, podría presentar mejores propiedades de transporte iónicas que la fase amorfa, debido a la morfología de las cadenas poliméricas. Los resultados además son consistentes con observaciones que indican que la inclusión de partículas cerámicas mejora la conductividad y el número de transporte iónico a causa de los efectos de interface.

En el caso de sistemas materiales no lineales la respuesta efectiva fue deducida de manera heurística a partir de un análisis de convergencia multi-escala de las ecuaciones de campo correspondientes. La respuesta efectiva homogenizada involucra un potencial efectivo por especie. Cada potencial es matemáticamente afín al de un conductor estándar no lineal. Una técnica de homogenización lineal de comparación es utilizada para generar las estimaciones de dichos potenciales no lineales en términos de las estimaciones disponibles correspondientes a los conductores lineales. A manera de ejemplo, se hace uso de las aproximaciones lineales de Maxwell-Garnet y del Medio Efectivo para generar estimaciones para sistemas bifásicos con disipaciones del tipo ley de la potencia. Fórmulas explícitas son presentadas para algunos casos límite. En el caso de respuestas del tipo umbral, las estimaciones presentan límites diluidos no analíticos y serían consistentes con campos localizados en caminos de mínima energía. 


\section{Capítulo 1}

\section{Introducción}

El transporte iónico en sólidos ha sido objetivo de numerosos trabajos de investigación en los últimos 40 años ya que sus potenciales aplicaciones tecnológicas brindarían ventajas sustanciales en diversos sistemas.

Las baterías convencionales de ion-litio consisten en un cátodo de un óxido o sulfuro metálico, un ánodo de grafito o silicio, y un electrolito líquido intercalado entre ambos que permite el flujo de iones de litio pero impide el flujo de electrones. La posibilidad de reemplazar el electrolito líquido por un electrolito sólido resulta sumamente atractiva por cuanto la ausencia de una fase líquida mejora la seguridad, durabilidad y confiabilidad de la batería y simplifica su diseño (ej., Teofilo \& Nadel 1998). Más aún, la batería de estado sólido así obtenida puede tener capacidad portante suficiente como para oficiar de fuente de potencia y de elemento estructural simultáneamente, acuñando así el concepto de "batería estructural". La posibilidad de combinar estas dos funciones en un solo elemento resulta sumamente atractiva en aplicaciones donde es crítico minimizar el peso o volumen estructural asociado a las fuentes de potencia eléctricas. Tal es el caso, por ejemplo, de vehículos aéreos no tripulados propulsados por motores eléctricos, en los que las baterías pueden representar una fracción considerable de la masa total y que podrían ser integradas en los perfiles aerodinámicos de los mismos (ej., Christodoulou \& Venables 2003), generando mayor volumen libre.

Bajo el mismo concepto, diversos diseños de satélites se podrían ver beneficiados inte- 
grando las baterías dentro de su estructura. De esta manera se reduciría o eliminaría por completo el peso de las estructuras parásitas que sólo cumplen la función de alojar la batería. Incluso para ciertas relaciones de peso del satélite y capacidad de las baterías (como en el caso de minisatélites -hasta 500 kg.--), las reducciones de peso podrían ser mayores aún (véase Roberts \& Aglietti 2007).

En el ámbito de las aplicaciones de uso masivo se puede citar el caso de los automóviles eléctricos, la electrónica de consumo y flexo-electrónica, que son algunos de los múltiples productos que podrían explotar las posibilidades que las baterías de estado sólido ofrecerían de estar disponibles en el mercado. En el caso puntual de los automóviles, por citar un ejemplo ya desde 2012 se están desarrollando prototipos en conjunto con Volvo Car Corporation (véase Asp et al. 2015) donde baterías multifuncionales integradas en el fondo del baúl y el techo, reemplazando paneles metálicos, podrían reducir desde un $15 \%$ el peso total del vehículo, aumentando su rango de alcance.

Las propiedades del electrolito sólido que más impactan en el desempeño de una batería son la conductividad iónica, la estabilidad electroquímica, la rigidez y la densidad. Dada esta multiplicidad de propiedades a optimizar, actualmente se desarrollan electrolitos cerámicos, poliméricos e incluso multifásicos (véase, por ejemplo, Syzdek et al. 2009, Fergus 2010, Funke 2013). Típicamente, estos electrolitos se fabrican en forma de films con espesores de entre $1 \mu \mathrm{m}$ y $500 \mu \mathrm{m}$ dependiendo del material (véase por ej. Latham \& Linford 1987, Tatsumisago et al. 2013).

El transporte de iones a través de electrolitos sólidos ocurre por el salto de iones (hopping) entre sitios de coordinación distribuidos por la estructura molecular (ej., Fritzsche \& Pollak 1990). Bajo intensidades de campo eléctrico bajas, este mecanismo de transporte produce un flujo de iones proporcional a la intensidad de campo. Para intensidades de campo moderadas, sin embargo, el flujo de iones se incrementa de manera no lineal con la intensidad de campo, generalmente siguiendo una ley de tipo seno hiperbólico (ej., Heuer et al. 2005). Finalmente, bajo intensidades de campo suficientemente altas se produce la rotura eléctrica del electrolito (ej., Sequeira \& Hooper 1992). En todos los casos, las propiedades de transporte dependen fuertemente de las características microestructurales del electrolito. En el caso de electrolitos cerámicos policristalinos, por ejemplo, el transporte de iones puede verse fuer- 
temente afectado por la presencia de precipitados intragranulares, inclusiones de segundas fases, porosidad, e incluso variaciones composicionales a través de los granos (ej., Badwal \& Drennan 1994). En el caso de electrolitos poliméricos semicristalinos, se suma la morfología microestructural (esferulítica, laminar, etc.) que adoptan las fases amorfas y cristalinas (ej., Minami et al. 2005, Marzantowicz et al. 2008, Fullerton-Shirey \& Maranas 2009). Las escalas de longitud características de estas heterogeneidades microestructurales pueden variar desde algunos nanómetros hasta algunos micrómetros. A pesar de ello, cambios moderados en algunas ellas pueden producir cambios pronunciados en las propiedades de transporte iónico. Por ejemplo, la conductividad iónica de óxidos de polietileno dopados puede incrementarse órdenes de magnitud cuando su microestructura laminar se reorienta por deformación (Golodnitsky \& Peled 2000). En consecuencia, la conductividad iónica de un electrolito sólido puede variar significativamente cuando las condiciones bajo las cuales se procesa o se utiliza favorecen variaciones microestructurales. El desarrollo tanto de la ciencia como de la técnica de electrolitos sólidos requiere pues teorías constitutivas que correlacionen las propiedades de transporte iónico con las características microestructurales subyacentes. El presente trabajo tiene como principal objetivo desarrollar un formalismo teórico que permita determinar tales correlaciones en electrolitos donde las escalas de longitud características de las heterogeneidades microestructurales relevantes son mucho menores que las dimensiones características del sistema.

Se requiere pues un formalismo capaz de describir fenómenos de transporte iónico no lineales a través de medios microestructurados con múltiples escalas espaciales. Las teorías multiescala propuestas hasta el momento asumen, invariablemente, una relación lineal entre el flujo de iones y la intensidad de campo eléctrico. Más aún, la mayor parte de las estimaciones disponibles (ej., Siekierski \& Nadara 2005, Belova \& Murch 2005, Kalnaus et al. 2011) emplean descripciones muy elementales del fenómeno de transporte (ej., ley de Ohm) y de las heterogeneidades materiales (ej., redes de resistores aleatorias). El formalismo aquí propuesto permite incorporar descripciones realistas tanto del fenómeno de transporte como de las heterogeneidades materiales. La derivación se apoya en la física de medios continuos y en métodos variacionales de homogenización asintótica junto con métodos de linearización basados en el concepto de "medio lineal de comparación". Rigurosos métodos de homogeniza- 
ción asintótica ya han sido utilizados para estudiar celdas microestructuradas con electrolitos líquidos por Allaire et al. (2010) y Schmuck \& Bazant (2015), y métodos de linearización basados en "medios lineales de comparación" de complejidad creciente ya han sido utilizados para estudiar la conductividad eléctrica en sistemas no lineales por Ponte Castañeda (1992, 2001) y Kailasam \& Ponte Castañeda (1997). Estos trabajos constituyen pues un punto de partida para el presente.

La exposición del trabajo comienza en el Capítulo 2 con un caso de estudio experimental que pone en evidencia la gran influencia que puede tener la microestructura de un electrolito sólido en la conductividad iónica. En el Capítulo 3 se propone el formalismo teórico general, válido para electrolitos sólidos constituidos por un número arbitrario de fases capaces de conducir un número arbitrario de especies iónicas, introduciendo las principales hipótesis y derivando las restricciones constitutivas impuestas por la termodinámica de medios continuos. El Capítulo 4 considera el caso particular en el que el transporte iónico obedece una ley lineal. Allí se asume que la microestructura exhibe múltiples escalas espaciales características y se utiliza un método de convergencia multiescala para homogenizar el sistema de ecuaciones diferenciales pertinente. Se obtiene así un formalismo teórico multiescala para el transporte iónico a través de electrolitos sólidos microestructurados. Dicho formalismo se utiliza en el Capítulo 5 a modo de ejemplo para construir un modelo bidimensional de un óxido de polietileno dopado cuyas predicciones permiten evaluar diversas hipótesis acerca de las propiedades de transporte de las fases amorfas y cristalinas. El formalismo del Capítulo 4 se generaliza luego en el Capítulo 6 para leyes de transporte iónico no lineales siguiendo un razonamiento heurístico. El formalismo no lineal resultante se utiliza en el Capítulo 7 para derivar estimaciones de las propiedades de transporte iónico en sistemas materiales de tipo matriz-inclusión y granulares. Para ello se emplea una linearización basada en el concepto de 'medio lineal de comparación' de tipo secante generalizada. Asumiendo una ley potencial para el transporte iónico se evalúan las capacidades de dicha linearización para lidiar con respuestas fuertemente no lineales. Finalmente, en el Capítulo 8 se exponen las principales conclusiones del trabajo y las perspectivas futuras del programa aquí iniciado. La exposición contiene, a su vez, un apéndice en el que se proveen los resultados de las mediciones que permitieron obtener los resultados del Capítulo 2. 


\section{Capítulo 2}

\section{Caso de Estudio}

\section{1. Óxidos de polietileno dopados}

Los materiales poliméricos constituyen una opción particularmente atractiva para la fabricación de electrolitos sólidos debido a su baja densidad, facilidad de procesamiento y bajo costo de fabricación. A su vez, la flexibilidad característica de los polímeros permite que el electrolito acompañe las deformaciones de los electrodos producidas por la litiación e incluso representa una gran ventaja para posibles aplicaciones en flexo-electrónica.

El polímero más estudiado hasta el momento para baterías de iones de litio es el óxido de polietileno - PEO por sus siglas en inglés - dopado con una sal de litio como el ioduro o el perclorato de litio. Otros ejemplos incluyen el polivinilideno fluorado, el poliuretano y copolímeros como el poliacrilonitrilo. Todos ellos pueden emplearse tanto en su forma monolítica como reforzados, por ejemplo, con partículas cerámicas micrométricas.

A temperatura ambiente estos polímeros son semi-cristalinos, usualmente presentan microestructuras esferulíticas (véase, por ejemplo, Robitaille \& Fauteux 1986, Marzantowicz et al. 2008, Fullerton-Shirey \& Maranas 2009) y tienen la capacidad de disolver las sales de litio y separarlas en catión y anión para propiciar el mecanismo de hopping. Las fases amorfas y cristalinas se combinan espacialmente de diversas maneras dependiendo de la constitución del material y del proceso de fabricación — véase las Figuras 2.1 y 2.2. Estas dos 


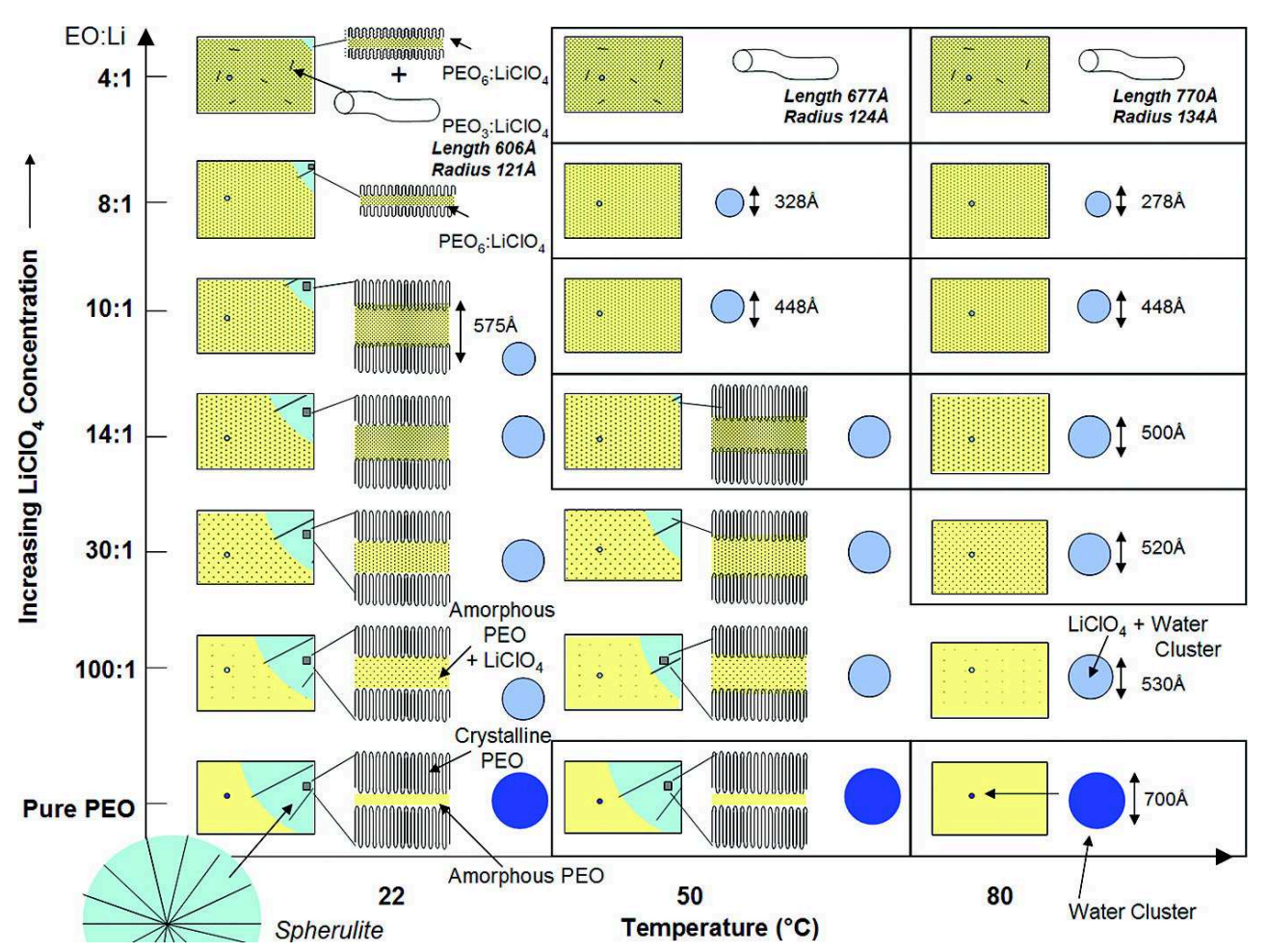

Figura 2.1: Esquema de la variación de la microestructura del PEO con la temperatura y la concentración de $\mathrm{LiClO}_{4}$ (extraído de Fullerton-Shirey \& Maranas 2009).

fases poseen propiedades mecánicas e iónicas distintas. Las propiedades macroscópicas del polímero dependen pues de las propiedades locales de las fases y de su distribución espacial: la microestructura. Estas características dependen a su vez de la temperatura.

Una gran parte de los estudios sobre electrolitos poliméricos se concentran en la variación de la conductividad iónica con la temperatura. La Figura 2.3-a muestra resultados en óxidos de polietileno dopados con diversas sales de litio. La conductividad aumenta con la temperatura debido al efecto que ésta produce en el mecanismo de interacción ion-macromolécula — véase, por ejemplo, Heumen \& Stevens (1995). Se observa que al variar la temperatura de $20^{\circ} \mathrm{C}$ a $60^{\circ} \mathrm{C}$ la conductividad aumenta en dos órdenes de magnitud.

Como la proporción de fase cristalina disminuye con la temperatura, los trabajos pioneros de la época moderna sobre estos materiales han expuesto un notable aumento de las propiedades de transporte iónico al incrementar la temperatura, fenómeno siempre acompañado por una considerable reducción en la cristalinidad (Berthier et al. 1983). Estas observaciones fomentaron las hipótesis que aseguran que es la fase amorfa la principal responsable de determinar las propiedades de transporte iónico en pólímeros semicristalinos. Debido a ello, 
a)

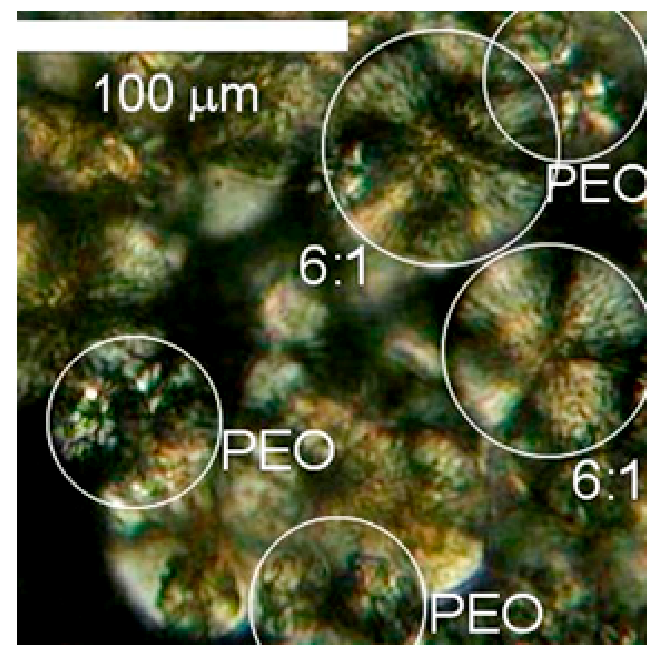

b)

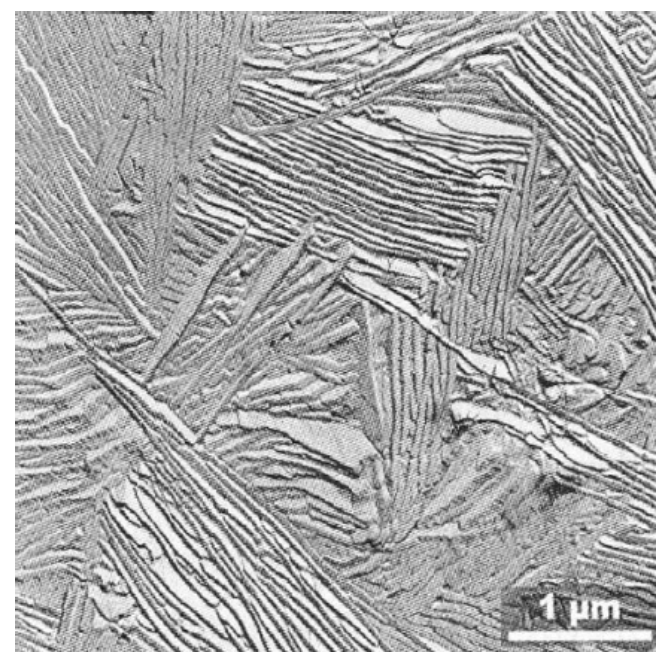

Figura 2.2: Microestructuras de polímeros semi-cristalinos a temperatura ambiente: a) estructuras esferulíticas de un PEO dopado con perclorato de litio (extraído de Marzantowicz 2008), b) estructura granular en un polietileno cristalizado (extraído de Boudet 2003).

casi la totalidad de los trabajos se han centrado en generar complejos poliméricos con la mínima cristalinidad posible -véase Funke (2013).

Sin embargo, estudios relativamente recientes han demostrado que la conductividad del PEO puede incrementarse varios órdenes de magnitud cuando los especímenes son sometidos a largas deformaciones (Golodnitsky \& Peled 2000, Golodnitsky et al. 2001, Minami et al. 2005). La Figura 2.4 muestra resultados para PEO dopado con $\mathrm{LiClO}_{4}$. Estas observaciones sugieren que probablemente sea la fase cristalina, altamente anisótropa, la que tal vez pueda ofrecer mejores propiedades de transporte, a lo largo de las direcciones alineadas con los ejes de deformación, incluso por encima de los valores de conductividad de la fase amorfa. Cabe recordar que, el PEO cristalino consiste de celdas unitarias monoclínicas conformadas por cadenas helicoidales, las cuales favorecen el transporte iónico a lo largo del eje del helicoide (véase Gitelman et al. 2008). Mediciones por difracción de rayos X sugieren que este acoplamiento mecánico-iónico es resultado de la reorientación de las cadenas poliméricas, tanto en las fases amorfas como en las cristalinas, inducida por la deformación aplicada — véase la Figura 2.5. Finalmente, todo esto suguiere que se podrían conseguir complejos de PEO y litio altamente cristalinos adoptando la estrategia de modificar la textura crsitalográfica, reemplazando el enfoque habitual centrado en reducir la cristalinidad. 
a)

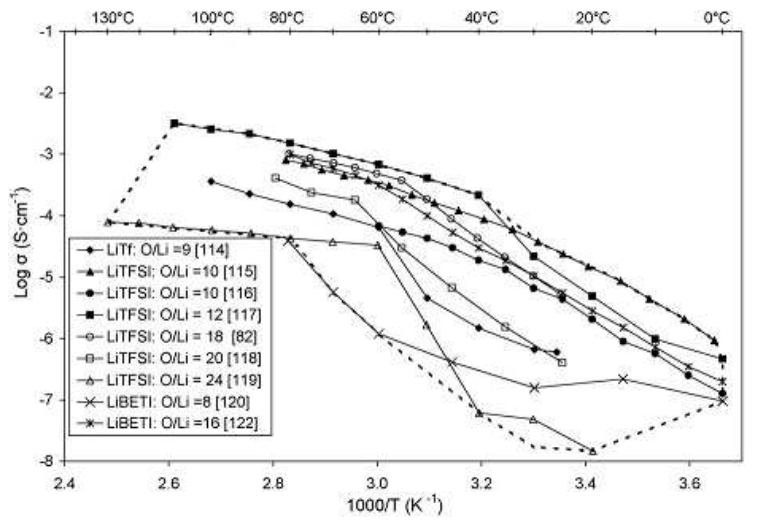

b)

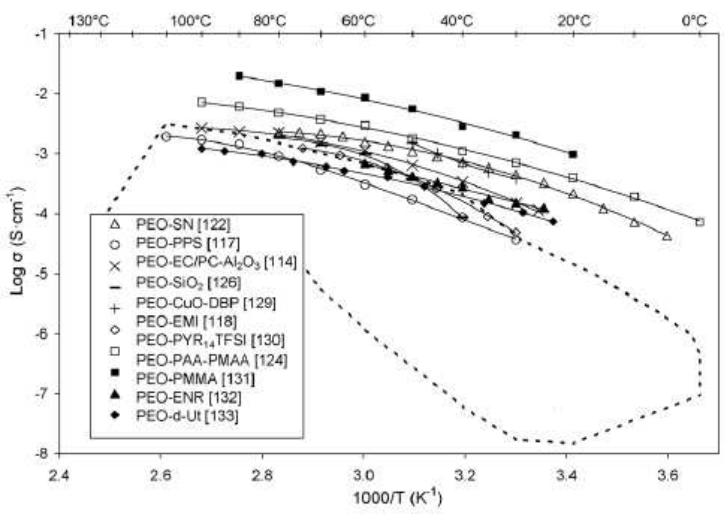

Figura 2.3: Conductividad iónica del PEO como función de la temperatura: a) PEO con diversas sales de litio, b) PEO con diversos plastificadores y partículas cerámicas. Extraído de Fergus (2010).

Paralelamente, estudios sobre diferentes compuestos poliméricos han revelado que tanto la conductividad iónica como el número de transporte catiónico pueden aumentar al agregar partículas cerámicas a los complejos de PEO y litio, por ejemplo óxido de aluminio $\left(\mathrm{Al}_{2} \mathrm{O}_{3}\right)$ o dióxido de zirconio $\left(\mathrm{ZrO}_{2}\right)$ —véase Croce et al. (2001) y (2006), Ciocek et al. (2007), Capuano et al. (1991). La Figura 2.3-b muestra la conductividad iónica de PEOs con diversos cerámicos $-\mathrm{CuO}, \mathrm{SiO}_{2}, \mathrm{Al}_{2} \mathrm{O}_{3}-$ y otros aditivos poliméricos. La línea de puntos denota la envolvente de las curvas mostradas en la Figura 2.3-a. Se observa que la conductividad iónica de los compuestos es superior a la del PEO monolítico y adopta valores cercanos a $10^{-3} \mathrm{~S} / \mathrm{cm}$ a temperatura ambiente, nivel mínimo habitualmente requerido en aplicaciones tecnológicas. El contenido de partículas óptimo dependerá de la aplicación espécifica, ya que también se ven afectadas la rigidez y la densidad del electrolito. La rigidez de los compuestos de PEO es mayor a la del PEO monolítico - lo cual puede ser beneficioso para algunas aplicaciones pero perjudicial para otras tales como las baterías flexibles - pero también lo es la densidad.

Si bien las partículas cerámicas son aislantes iónicas, dicho incremento ha sido explicado a través del efecto que introducen las interfases entre las partículas y el polímero, donde se generan zonas amorfas que proveen de "anclajes" químicos a los aniones. Dicha hipótesis gana fuerza al verificar que el tratamiento superficial de las partículas introducidas tiene un gran impacto en las propiedades de transporte del compuesto polimérico (Croce et al. 2001). En cualquier caso, la presencia de partículas en el compuesto incorpora otra variable microestructural que deberá tenerse en cuenta en el análisis micromecánico de electrolitos 

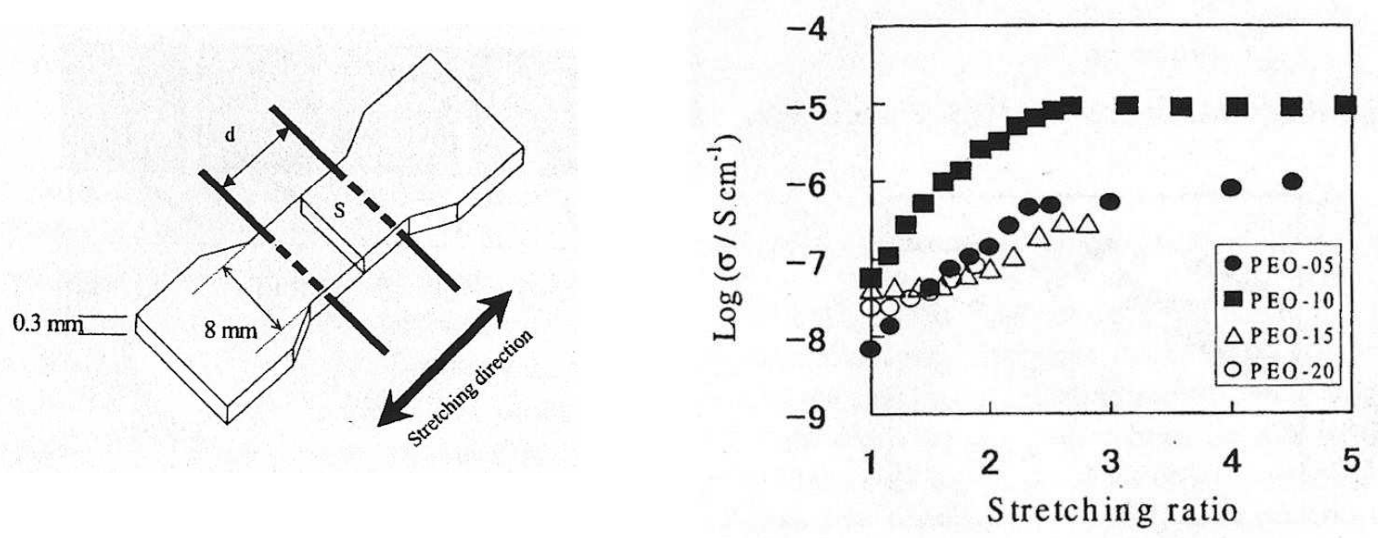

Figura 2.4: Variación de la conductividad iónica longitudinal a temperatura ambiente con la deformación en PEO dopado con $\mathrm{LiClO}_{4}$. PEO- $n n$ corresponde a un PEO con $n n$ mol \% de sales. Extraído de Minami et al. (2005).

compuestos.
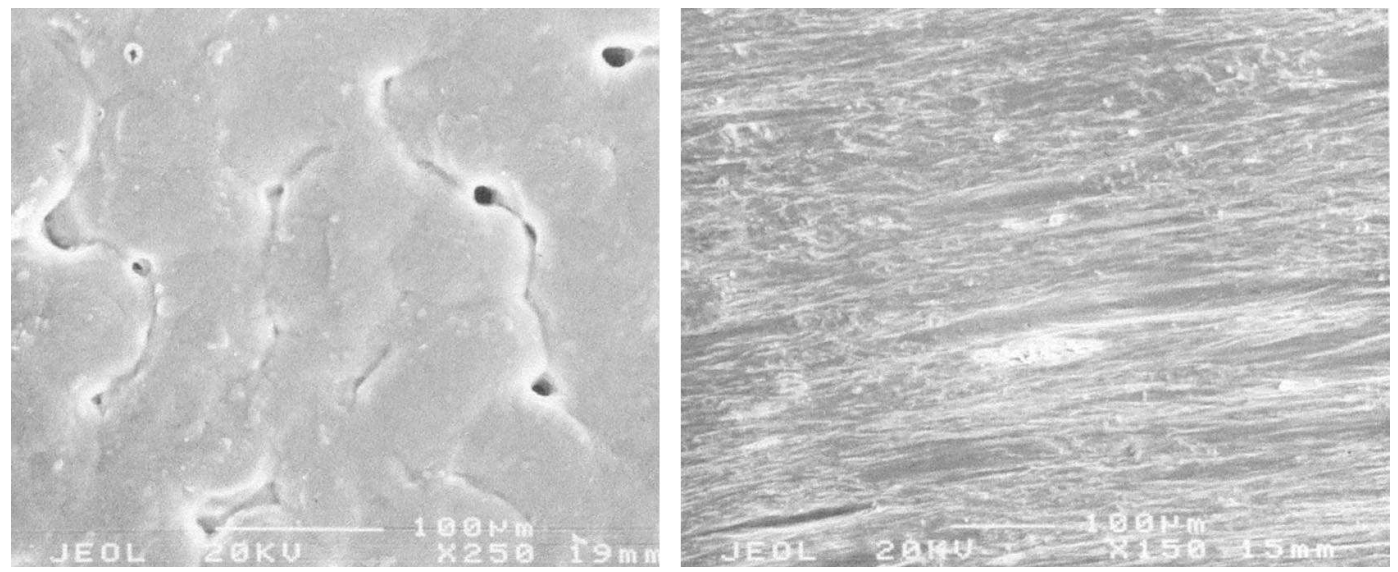

Figura 2.5: Microestructura de LiI-PEO no deformada (izquierda) y deformada (derecha). Extraído de Golodnitsky \& Peled (2000).

\subsection{Estudio experimental}

En virtud de lo expuesto se llevaron a cabo una serie de mediciones experimentales propias con el objetivo de correlacionar, al menos cualitativamente, la microestructura de estos polímeros semicristalinos con la conductividad iónica. Como sistema material de estudio se adoptó el óxido de polietileno (PEO, por sus siglas en inglés), dopado con sales de cloruro 
de litio $(\mathrm{LiCl})$. Los motivos de elección de dicho sistema son principalmente su relativa sencillez de fabricación y la numerosa información experimental disponible sobre el mismo.

La idea fundamental fue monitorear los cambios en la conductividad que pudieran aparecer por cambios en la microestructura. Dichos cambios fueron generados de dos maneras: por efecto del paso del tiempo y a causa de las diferentes concentraciones de sales de litio. Como ya veremos más adelante en la discusión de los resultados, sendos cambios impactan en la microestructura, modificando la cristalinidad y la forma en que las fases se organizan a nivel micro y meso estructural.

En líneas generales las probetas fueron obtenidas por el método de casteo y estudiadas microestructuralmente a través de la técnica de Calorimetría Diferencial de Barrido (DSC, por su siglas en inglés) con el objetivo de poder estimar su cristalinidad. Mediciones complementarias de cristalinidad fueron realizadas mediante la técnica de Difracción de Rayos X (XRD, por sus siglas en inglés). La conductividad iónica fue obtenida mediante ensayos de conductividad en corriente continua, realizados con una adaptación del método de las cuatro puntas particularmente desarrollado para el presente trabajo.

Las propiedades de las probetas son medidas al momento de su desmolde, es decir al ser fabricada, luego dejadas envejecer a temperatura y humedad controlada con el objetivo de permitir que su microestrcutura evolucione hacia un estado más ordenado y por ende su cristalinidad aumente. Luego de ciertos intervalos de tiempo de espera, la conductividad es nuevamente medida y la calorimetría diferencial de barrido es nuevamente efectuada, a modo de poder cuantificar ese cambio en la microestrctura. Teóricamente la microestructura deberá ser cada vez más cristalina con el pasar del tiempo y la conductividad iónica debería descender, en concordancia con la mayoría de las observaciones experimentales que están disponibles en la literaura de referencia.

Particularmente las probetas fueron fabricadas con concentración molar de PEO:litio de 10:1, 20:1 y 30:1. Sobre todas ellas se midió la conductividad a lo largo del tiempo. Se fabricaron 3 probetas de cada concentración, de las cuales se extrajeron 3 rectángulos de cada una para medir la conductividad y una numerosa cantidad de trozos menores para realizar los barridos de DSC. El monitoreo de la evolución de la microestructura fue cuantificado a través de los barridos de DSC, los cuales por una cuestión de recursos y de tiempos de 
análisis, fueron realizados solamente sobre las probetas de concentración 20:1. La elección de las mismas fue debido a que se cuenta con más datos experimentales en materiales de esta concentración de litio y eso facilita poder comparar los valores obtenidos.

\subsection{Obtención de las películas poliméricas}

Para obtener películas de PEO se utilizó el método de casteo (véase, por ejemplo, "Polymer Electrolytes: Fundamentals and Applications", Sequeira \& Santos, 2010), en el cual se disuelven el polímero y las sales en las proporciones deseadas utilizando un solvente. Luego, dicha solución se vierte sobre moldes, donde se permite que el solvente se evapore, dejando los sólidos unidos. En este proceso las cadenas poliméricas se "distienden" en la solución permitiendo la unión de sus terminales con las sales de litio, ya que el óxido de polietileno tiene la propiedad de separar las sales de litio en anión y catión. Luego, permitiendo que el solvente se evapore lentamente, las cadenas poliméricas van ganando un cierto ordenamiento que da como resultado estructuras semicristalinas esferulíticas.

El óxido de polietileno se obtuvo de la empresa Sigma-Aldrich con un peso molecular $M_{v}=600000$, y fue utilizado en forma de polvo. El cloruro de litio, también en formato en polvo, fue obtenido de la empresa Anedra. El solvente utilizado fue acetonitrilo grado HPLC $99.9 \%$ de la empresa J.T. Baker.

Previo a su uso, tanto el PEO como el cloruro de litio fueron secados al vacío durante 24 horas, a una temperatura de $45^{\circ} \mathrm{C}$ y $120^{\circ} \mathrm{C}$ respectivamente, de modo de extraer la humedad que pudieran contener. Una vez finalizado el secado, cada componente fue disuelto en acetonitrilo. El PEO y el cloruro de litio fueron disueltos en una proporción 1/10 en acetonitrilo, por separado, dejando las soluciones en agitación (mediante agitadores magnéticos) durante 48 horas, para asegurarse la homogenización de la misma. Luego, cada solución fue sometida durante 15 minutos a un agitador ultrasónico, como etapa final de la homogenizacion. Dentro de los recipientes donde se realizaron las soluciones se barrió el aire utilizando nitrógeno y fueron cerrados herméticamente, con el objetivo de generar una atmósfera inerte y minimizar la presencia de humedad. 


\begin{tabular}{||c|c|c||}
\hline Probeta & Moles de PEO:Moles de Li & Espesor promedio $[\mu m]$ \\
\hline \hline $10: 1-1$ & $10: 1$ & 228 \\
$10: 1-2$ & $10: 1$ & 226 \\
$10: 1-3$ & $10: 1$ & 224 \\
$20: 1-1$ & $20: 1$ & 230 \\
$20: 1-2$ & $20: 1$ & 195 \\
$20: 1-3$ & $20: 1$ & 196 \\
$30: 1-1$ & $30: 1$ & 180 \\
$30: 1-2$ & $30: 1$ & 154 \\
$30: 1-3$ & $30: 1$ & 192 \\
\hline
\end{tabular}

Tabla 2.1: Nomenclatura y espesor promedio de las probetas casteadas.

El molde utilizado para castear las probetas fue fabricado específicamente para este trabajo sobre una placa de politetrafluoroetileno (Teflon); en el mismo se mecanizaron rectángulos de diversos tamaños (véase la Figura 2.6-b) con una profundidad de 3 milímetros, suficiente para albergar un volúmen de solución que permita la evaporación del solvente, dejando la cantidad de sólido buscada para obtener películas del espesor deseado. Debido a la alta dilución requerida por el PEO en acetonitrilo para poder asegurar una correcta homogenización de la solución es necesario realizar varias adiciones de solución al molde a medida que el solvente evapora, para poder obtener películas del espesor buscado y que sean sencillas de manipular. El objetivo era obtener películas de 200 micrones de espesor, en promedio.

El procedimiento de casteo de las soluciones sobre el molde fue realizado dentro del ambiente controlado del laboratorio donde el molde fue dispuesto dentro de una caja de acrílico, a fin de minimizar la posibilidad de contaminación o corrientes de aire sobre las soluciones. A lo largo de cada día de casteo se fue vertiendo solución cuatro veces en cada molde, de manera espaciada, permitiendo que el solvente evapore lentamente entre adición y adición. De esa manera se obtuvieron películas de un espesor promedio de 203 micrones. Las mediciones de espesor fueron realizadas con un micrómetro marca Mitutoyo y se presentan en la tabla 2.1. 
a)

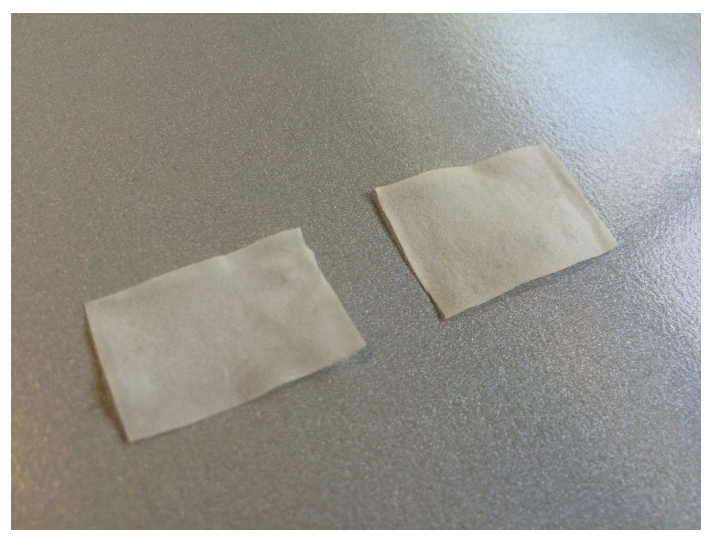

b)

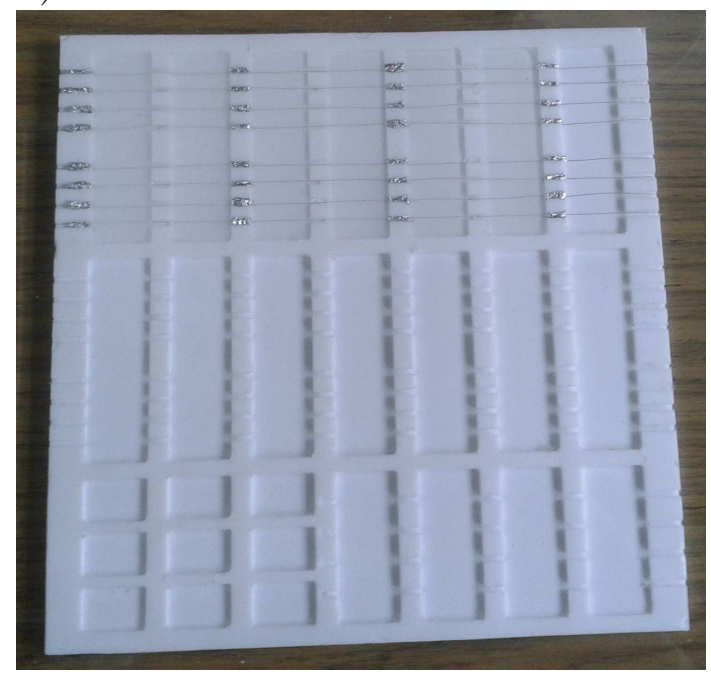

Figura 2.6: a) Imágenes de las películas casteadas, cada una mide 2.7 x $1.7 \mathrm{~cm}$ aproximadamente. b) Molde de teflon. En la parte superior se pueden observar los hilos de titanio asegurados en el molde y las primeras 6 divisiones contando desde la izquierda están llenas con PEO. El ancho de cada división es de $3 \mathrm{~cm}$.

\subsection{Medición de conductividad iónica}

Para poder realizar las mediciones de conductividad iónica, el método elegido fue el de cuantro puntas de corriente continua (véase, por ejemplo, Blythe \& Bloor 2005). Dicho método permite de forma rápida y directa obtener mediciones con un sencillo postproceso de los resultados. Antes de pasar a la explicación del método, cabe enfatizar que no fue el objetivo de este trabajo obtener el valor exacto de la conductividad de cada probeta, para lo cual el método de cuatro puntas de corriente continua no es el más apropiado. En el caso de querer obtener valores absolutos de conductividad el método de impedancia espectroscópica hubiese sido el correcto. Como el interés fue sólo estudiar el cambio en dicha propiedad y el método de cuatro puntas es directo y de menor costo (tanto de equipamiento como de postproceso), este último prevaleció en la elección.

Escencialmente, el método de cuatro puntas de corriente continua consta de cuatro puntos de conexión sobre la probeta a ser ensayada. Entre dos de estos puntos se establece una corriente fija y entre otros dos puntos se mide la caída de potencial. Con las medidas geométricas de la probeta y el valor de caída de potencial, se puede calcular el valor de conductividad (o resistencia) de la misma. Para esta tesis se evaluaron tres métodos de me- 
dición que se detallarán a continuación; a modo de referencia los llamaremos: método clásico, método de conexiones embebidas y método de placa de cobre.

El método clásico consta de cuatro conexiones independientes, dos de ellas conectadas a una fuente de corriente continua y otras dos a un multímetro de alta impedancia de entrada. El objetivo de cada punta es hacer contacto (idealmente) en un solo punto de la probeta. El orígen de dicho método se debe a la utilización de las puntas clásicas de los instrumentos de medición eléctrica. En este caso se probaron las puntas que vienen de fábrica en los instrumentos utilizados pero la metodología se volvía poco práctica al momento de mantener la corriente aplicada durante un tiempo prolongado. Como una evolución del método clásico se reemplazó las puntas por elementos metálicos de alta conductividad con una punta afilada. Dichas puntas fueron fijadas en un acrílico aislante a una distancia fija entre ellos, a modo de simplificar la operación de contacto entre las 4 puntas y la probeta. Este método clásico fue descartado luego de las primeras pruebas, principalmente debido a que no es sencillo asegurar el contacto correcto entre las puntas y la probeta, además debido a que al reiterar los ensayos las probetas se veían dañadas, sobre todo con las segundas puntas utilizadas, a causa del filo del extremo en contacto. Pero más allá de la dificultad práctica, el método no asegura una circulación de la corriente de manera homogénea a lo largo de la probeta, ya que son sólo puntos donde se aplica la corriente y por ende es de esperar que las líneas de corriente tiendan a converger a los puntos de aplicación. Esto podría falsear las medidas de conductividad donde se espera que el frente de avance de la corriente sea parejo en todo el ancho de la muestra. Además se hace imposible cuantificar qué porción de la corriente circula por el interior de la probeta respecto a las corrientes que puedan establecerse a lo largo de las superficies.

El método de conexiones embebidas fue ideado especialmente para este trabajo, basándonos en métodos similares (véase Golodnitsky \& Peled, 2000). Básicamente en este esquema se incluyen "dentro" de la probeta, durante su fabricación, cuatro filamentos conductivos que serán las "4 puntas" del método. Esto permite distribuir mejor en todo el ancho de la película la zona de aplicación de la corriente y por ende tender a un esquema más parejo de líneas de corriente. En la referencia citada, los terminales conectores quedaban 
a)

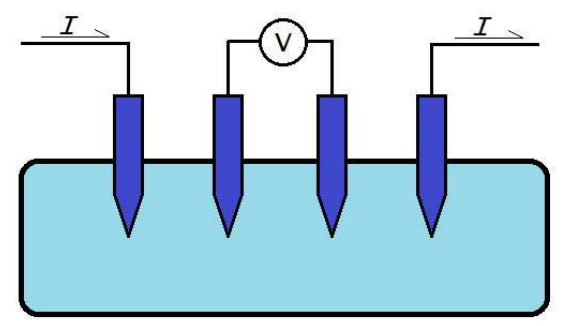

b)

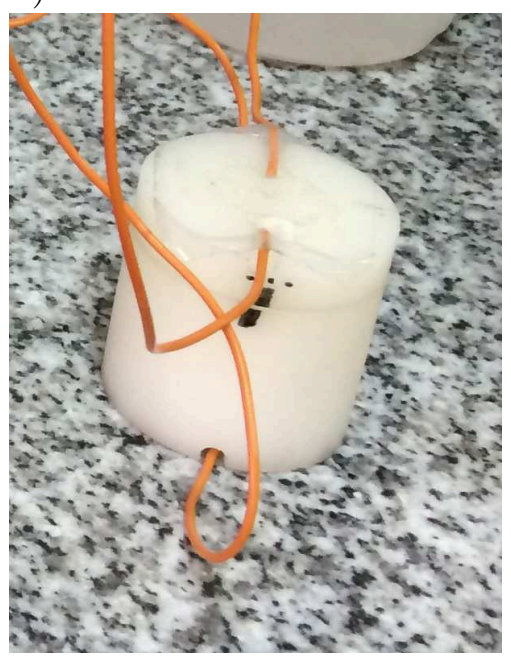

Figura 2.7: a) Esquema del método de las cuatro puntas. b) Dispositivo de teflon para ensayo de conductividad de cuatro puntas.

incluídos en el medio de dos probetas previamente fabricadas y prensadas en caliente para poder unirlas. Esta práctica fue descartada debido a que al prensar en caliente las probetas, se induce un cambio en la microestructura no deseado para el tipo de caracterización llevado a cabo. Para evitar esto es que se diseñó un molde en el cual fuera posible situar los filamentos conductivos previamente al casteo de las probetas, como se puede obsrevar en la Figura 2.6-b. Los alojamientos longitudinales donde se apoyan los filamentos están elevados del fondo del molde de la probeta unos 100 micrones, a efectos de dejarlos aproximadamente en el medio de las probetas de 200 micrones que se fabricarían con dicho molde. En la práctica el proceso de asegurar los filamentos representó una cierta dificultad de armado, pero se logró obtener películas del espesor esperado con los filamentos completamente embebidos en las mismas. Como filamentos se evaluaron tres opciones: cobre, aluminio y titanio. Los primeros dos, en los espesores necesarios para ser embebidos en un film de 200 micrones de espesor, presentaban una resistencia mecánica muy baja y tendían a cortarse al asegurarlos en el molde o al manipularlos posteriormente. Por este motivo se decidió utilizar alambres de titanio debido a su mayor resistencia mecánica y resistividad eléctrica aceptable. Si bien la conductividad del titanio es muy inferior a la del cobre y a la del aluminio, se encuentra varios órdenes de magnitud por encima de la del PEO dopado con litio, por ende no introduce un error apreciable en las mediciones. Este método arrojó varios resultados pero finalmente 
fue reemplazado por el método de placa de cobre ya que, como detallaremos, era de más sencilla ejecución y brindaba más confiabilidad sobre la consistencia de los resultados.

El método de placa de cobre está basado en el método de las cuatro puntas, para el cual se dispuso de dos placas planas de cobre de 1.5 x $1.5 \mathrm{~cm}$, pulidas, del mismo tamaño entre sí e idealmente del mismo tamaño de las probetas a ensayar. Dichas placas de cobre tienen dos pares de conexiones, las cuales como en el método de cuatro puntas, son dos para establecer la diferencia de potencial eléctrico y las otras dos para medir la corriente (o caída de potencial). Particularmente para este caso, cada placa de cobre tiene una conexión de cada tipo (una para establecer la diferencia de potencial y una de medición); debido a la alta conductividad del cobre, sobre todo respecto a la de las probetas en ensayo, se considera que cada placa está a un equipotencial y por ende que la medición de corriente (o caída de potencial) se está realizando "en el mismo punto" en el cual se establece la diferencia de potencial, si bien físicamente están en diferentes lugares de cada placa. El diseño del instrumento se completa con dos soportes de politetrafluoroetileno (Teflon) que contienen cada placa de cobre y sus conexiones, para no comprometer la integridad de las mismas durante la manipulación y para aislar eléctricamente el ensayo. Tanto el establecimiento de la diferencia de potencial como las mediciones de corriente (o caída de potencial) fueron realizadas con un microvoltímetro Keithley 197A.

El procedimiento de ensayo de cada probeta consiste en colocar un film del espécimen del tamaño de las placas de cobre y en contacto con las mismas, cerrando ambas partes de teflon, como se ve en la Figura 2.7. Luego se coloca $1 \mathrm{~kg}$ de peso sobre el sistema, con el objetivo de asegurar el contacto completo de la probeta con las placas de cobre y ahí se establece la diferencia de potencial deseada, momento en el cual comienza a correr el tiempo de ensayo. El potencial se deja aplicado durante 5 minutos. Los primeros 30 segundos de ensayo se dejan transcurrir a fin de descartar posibles transitorios y se comienza a adquirir el valor de resistencia cada 15 segundos hasta el final del tiempo prefijado. Debemos aclarar que el valor de conductividad considerado para los resultados es el promedio entre los valores adquiridos entre los 30 y 90 segundos del ensayo. Debido al método de medición elegido, se genera una polarización en la probeta ensayada que hace que la conductividad cambie con el tiempo, es decir, los iones se agrupan contra las superficies de las probetas, cercanas a las zonas de 
aplicación del potencial y por ende la cantidad de iones libres en el interior del material, capaces de transportar carga, disminuye. Esto hace que se evidencie una reducción de la conductividad durante el tiempo de medición. Estas afirmaciones se han verificado con otros arreglos del método de cuatro puntas, donde una vez eliminada la diferencia de potencial que se aplica, es posible medir una inversión de la corriente que circula por la probeta. Debido a este efecto, y recordando que el objetivo de dichas mediciones es evidenciar cambios en la conductividad con el paso de los días, se ha elegido considerar como valor de conductividad el promedio del lapso indicado, para todos los ensayos por igual.

\subsection{Medición de la cristalinidad}

\subsubsection{Calorimetría Diferencial de Barrido}

La técnica de Calorimetría Diferencial de Barrido (DSC) permite medir la diferencia de energía que se requiere para calentar una determinada cantidad de grados un espécimen en estudio comparado con una referencia conocida. De esta manera es posible obtener, por ejemplo, la energía necesaria para la transición de sólido a líquido de un material y a qué temperatura ocurre dicho evento. Para ello, una muestra del matrerial en estudio y una referencia de capacidad calorífica conocida, se someten a un barrido dinámico en un rango de temperatura prefijado durante el cual se registra el intercambio de energía que se requiere para mantener ambos materiales a la misma temperatura a lo largo de todo el barrido.

En este trabajo se realizaron barridos de DSC sobre las probetas de óxido de polietileno dopadas con sales de cloruro de litio con el fin de conocer la energía requerida para lograr que la probeta se funda y pase de estado sólido a líquido. Conocida la energía necesaria para esta transformación de fase y comparándola con la energía necesaria para fundir el mismo polímero con una microestructura $100 \%$ cristalina, se puede estimar el porcentaje de cristalinidad de la probeta bajo análisis. Conocer la cristalinidad de cada probeta nos permite tener un parámetro microestructural que resulta fundamental en la correlación microestructura-propiedades de transporte. 


\begin{tabular}{||l|l||}
\hline Parámetro & Rango o descripción \\
\hline \hline Rango de temperatura & $22-120^{\circ} \mathrm{C}$ \\
Masa de la muestra & $5-6.5 \mathrm{mg}$ \\
Flujo de $\mathrm{CO}_{2}$ & $20 \mathrm{ml} / \mathrm{min}$ \\
Cápsula porta muestra & Aluminio con tapa \\
Referencia del equipo & $\mathrm{Al}_{2} \mathrm{O}_{3}$ \\
Peso de la referencia del equipo & $4 \mathrm{mg}$ \\
\hline
\end{tabular}

Tabla 2.2: Rangos utilizados en los análisis de DSC

Así como en el caso de las mediciones de conductividad, los análisis de calorimetría también requirieron de una calibración previa. Durante estas pruebas preliminares se pudo establecer una línea de base del equipo que sea confiable y permita correcciones posteriores; se pudo estimar la cantidad de masa óptima para conseguir resultados útiles a los fines de obtener la entalpía de fusión de la muestra y por ende poder calcular la cristalinidad de la misma y además se pudo determinar los rangos de temperatura útiles entre los cuales realizar el barrido. Además de todo esto, las pruebas preliminares permitieron generar una rutina de trabajo y manipulación de las muestras que arrojara valores repetitivos y verificables. Luego de un período de calibración y rediseño de los ensayos se concluyó en utilizar los parámetros que se detallan en la tabla 2.2. El equipo utilizado es un SHIMADZU DSC-60.

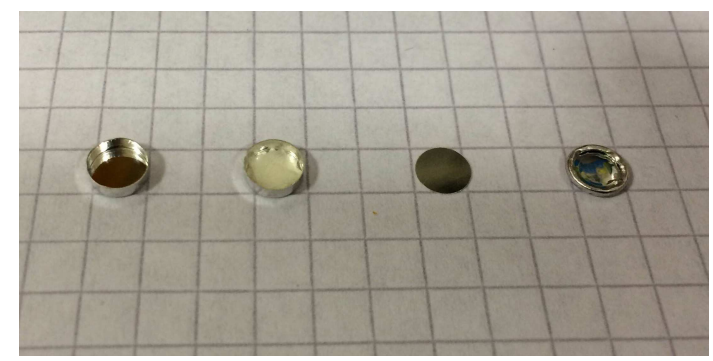

Figura 2.8: Cápsulas de aluminio utilizadas para contener la muestra. De izquierda a derecha: cápsula vacía, cápsula con película de $\mathrm{PEO}$ dentro, tapa de las cápsulas, cápsula con $\mathrm{PEO}$, cerrada con su tapa y sellada lista para ser introducida en el equipo de medición. El tamaño de cada división cuadriculada es de 5 $\mathrm{x} 5 \mathrm{~mm}$. 


\subsubsection{Preparación de muestras de DSC}

El proceso de preparación de las muestras para los barridos de calorimetría diferencial implica el pesaje preciso de la masa introducida dentro de las cápsulas porta muestra. Dicho dato es de vital relevancia para el cálculo de la entalpía de fusión por unidad de masa, que es el valor que permite estimar la cristalinidad de la muestra. Para ello se procedió a pesar cada una de las cápsulas a ser utilizadas con su respectiva tapa, rotularlas para poder considerar las pequeñas variaciones de masa que las mismas posean entre sí y luego pesar el espécimen a analizar. Todos los pesajes se realizaron por duplicado, con dos balanzas analíticas de precisión, ambas calibradas.

La cantidad de PEO que se introduce en cada análisis es del orden de los 5 miligramos. Dichos trozos fueron obtenidos de probetas casteadas en moldes más grandes, como fuera detallado previamente. Con el objetivo de muestrear representativamente cada ejemplar, se seleccionaron varios trozos de diversas partes de un mismo espécimen para cada medición, con el objetivo de realizar un muestreo más amplio del material en estudio. Los valores obtenidos por barrido calorimétrico son de alta precisión pero tienen la desventaja que se basan sólo en una muy pequeña porción del material en estudio. Para ampliar la representatividad de los resultados, es que se decidió "mezclar" diferentes partes de la misma probeta en cada muestra. Además se realizaron todos los análisis por duplicado para dos probetas iguales, casteadas en diferentes moldes, de manera de evidenciar posibles variaciones generadas por el proceso de casteo.

A fin de obtener una estimación del márgen de error del equipo, se decidió realizar dos corridas con dos porciones de una misma probeta de PEO puro, para establecer la cristalinidad de la misma y comparar los resultados. Para dicho ensayo se verificó una diferencia en la estimación de la cristalinidad de $0.3 \%$. Se adjuntan las curvas en el Apéndice.

\subsubsection{Difracción de Rayos X - XRD}

En el método de Difracción de Rayos X, explicado de manera resumida, se utiliza un haz monocromático de rayos-X para bombardear una muestra en estudio y luego se mide la 
intensidad de la dispersión de átomos producida en el material objetivo. Dicho análisis se realiza en función del ángulo de incidencia, lo cual permite evidenciar estructuras coherentes y por ende obtener información de la cristalinidad de una muestra, dato de particular interés para este trabajo. Además dicha técnica depende de la polarización y de la longitud de onda o energía del haz incidente. Es importante destacar que las direccciones de las posibles difracciones dependen del tamaño y de la forma de la celda unitaria del material en estudio mientras que las intensidades de las ondas de difracción dependen del tipo de empaquetamiento de los átomos en la estructura cristalina, arrojando de esta manera información de varios parámteros microestructurales.

Mas allá de toda la información que este método puede brindar, el único objetivo de incluir algunas mediciones en el presente trabajo de tesis fue el de obtener valores de la cristalinidad de algunas muestras para poder compararlas con las obtenidas por el método de calorimeteía diferencial, es decir a modo comparativo y complementario de las mismas.

Particularmente para los análisis de rayos $\mathrm{X}$ que se realizaron para este trabajo de tesis se cortaron probetas cuadradas tipo film de $2.5 \mathrm{~cm}$ de lado, fabricadas con la misma técnica que se explica en la sección 2.2 .

Las curvas obtenidas de estos análisis pueden observarse en el Apéndice.

\subsection{Resultados}

Se presentan a continuación los resultados de las mediciones de conductividad. En la Figura 2.9 se muestran los valores adquiridos y en la Figura 2.10 las variaciones porcentuales respecto a la conductividad inicial de cada espécimen de las dos concentraciones de litio diferentes, 10:1 y 20:1.

Los resultados arrojados por las mediciones de conductividad en general son consistentes con las hipótesis planteadas. Con el avance del tiempo, la microestructura de las probetas de PEO tienden a un estado más ordenado, más cristalino. Eso hace que de manera macroscópica, la conductividad iónica se reduzca y eso es lo que se evidenció en las mediciones de conductividad bajo corriente continua. 
a)

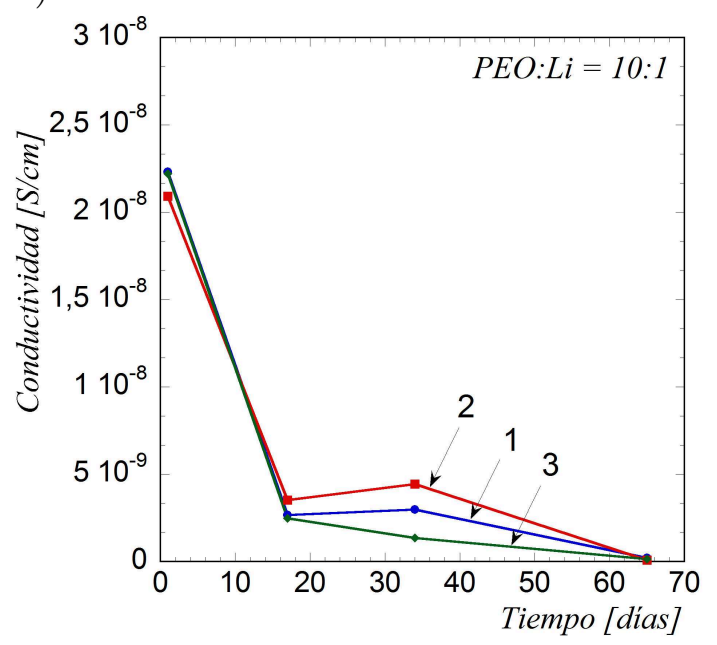

b)

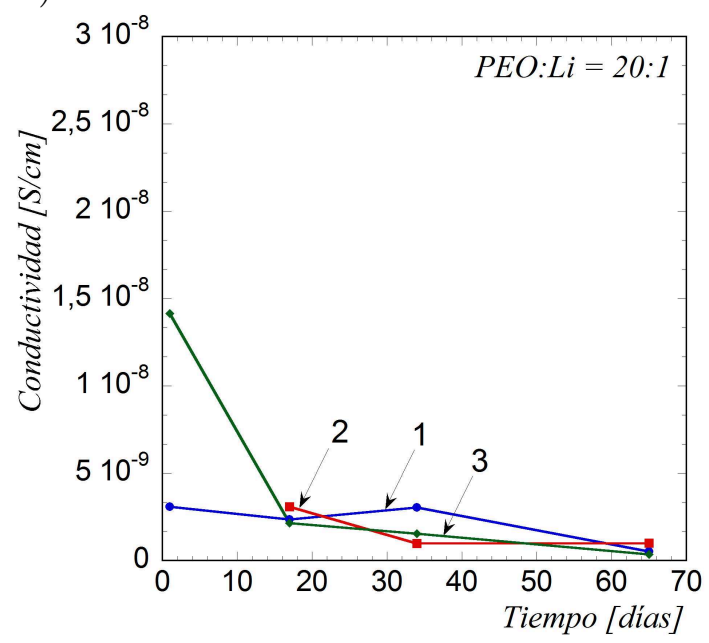

Figura 2.9: Mediciones de conductividad vs. tiempo a) películas de concentración PEO:Li=10:1 b) películas de concentración PEO: $\mathrm{Li}=20: 1$.

Se puede ver que los resultados de las muestras 10:1 presentan un comportamiento más acorde al esperado, si bien las probetas 20:1 también presentan un descenso en la conductividad con el avance del tiempo pero sus cambios son menores a primera vista. Sin embargo, al analizar los cambios porcentuales respecto a la cristalinidad inicial, en la Figura 2.10 se observa que el comportamiento es similar al de las de mayor concentración de litio. Es importante destacar que las mediciones de conductividad en las probetas de mayor presencia de litio se facilitan debido a que sus valores mayores de conductividad son más fáciles de adquirir y los errores son menores porcentualmente respecto a los valores absolutos que en las más diluídas.

Se presentan a continuación los resultados de cristalinidad obtenidos por Calorimetría Diferencial de Barrido para las muestras de concentración molar 20:1. La Figura 2.11-a presenta los valores de cristaliniad porcentual respecto al total de la muestra y la Figura 2.11-b presenta los mismos resultados pero como variación de la cristalinidad respecto a la cristalinidad inicial. Finalmente se presentan, en la Figura 2.12, los resultados comparativos de conductividad en función de la cristalinidad donde se agregan las mediciones de cristalinidad realizadas por el método de Difracción de Rayos X.

Las mediciones de calorimetría diferencial de barrido evidencian la alta heterogeneidad de las muestras. Esta heterogeneidad, por las conclusiones obtenidas en el presente trabajo, 
a)

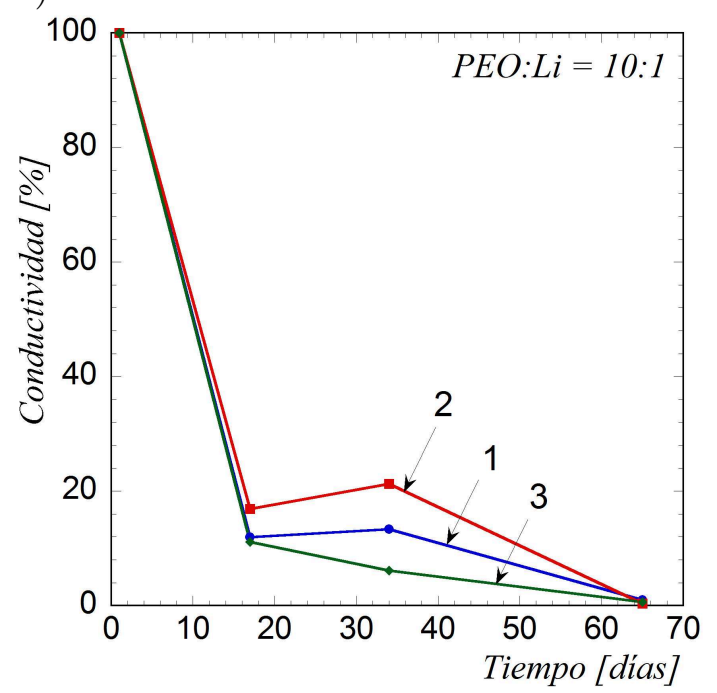

b)

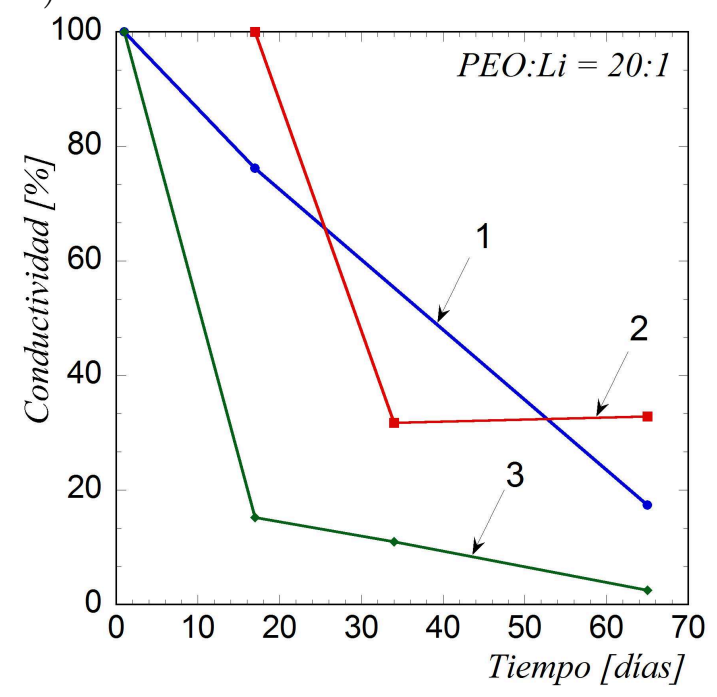

Figura 2.10: Mediciones variación porcentual de la conductividad vs. tiempo a) películas de concentración PEO:Li=10:1 b) películas de concentración PEO:Li=20:1.

provienen de dos fuentes. La primera es intrínseca al material en sí y se debe a la cinética de cristalización del PEO dopado con litio. Como ya hemos descripto, el PEO es un polímero semicristalino con una microestructura de dos escalas, la escala menor presenta una forma de laminado bifásico formado por zonas amorfas y zonas cristalinas; la escala superior es del tipo esferulítica, donde las láminas ya nombradas se distribuyen en forma radial formando los radios de dichas esferulitas. La presencia del litio genera puntos de nucleación donde se inician las formaciones de dichas esferulitas, pero a su vez baja, en promedio, el tamaño de las mismas (véase Fullerton-Shirey \& Maranas 2009). Esto último hace que la cristalinidad del PEO dopado sea menor que la del PEO puro. La segunda causa de la heterogeneidad proviene del proceso de casteo en sí mismo. En el proceso de fabricación de las probetas se forma una solución de PEO y una sal de litio que se homogeniza y luego se castea. Si bien se realiza una homogenización de la mezcla en su estado líquido, eso no garantiza que la sal de litio se distribuya de manera homogénea en el molde una vez casteado. Si, como ya se dijo, la presencia de sales de litio afecta la cristalinidad del material, y la distribución de litio no es pareja, es esperable que la microestructura presente un grado de heterogeneidad aún mayor. Eso se evidencia en las curvas de DSC, sobre todo cuando para un mismo ejemplar de PEO los picos del evento endotérmico ocurren a temperaturas diferentes, hecho que indica composiciones diversas de las muestras, fácilmente explicables por la diferencia de cantidad 
a)

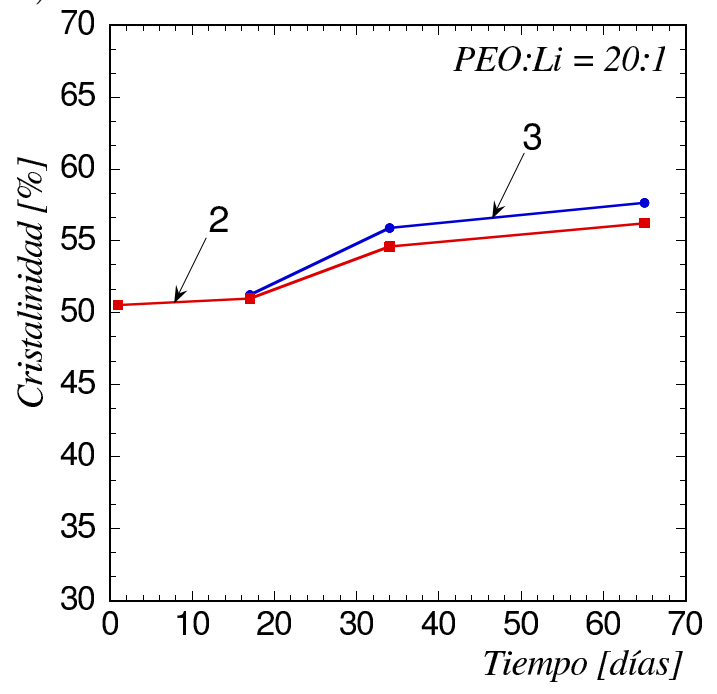

b)

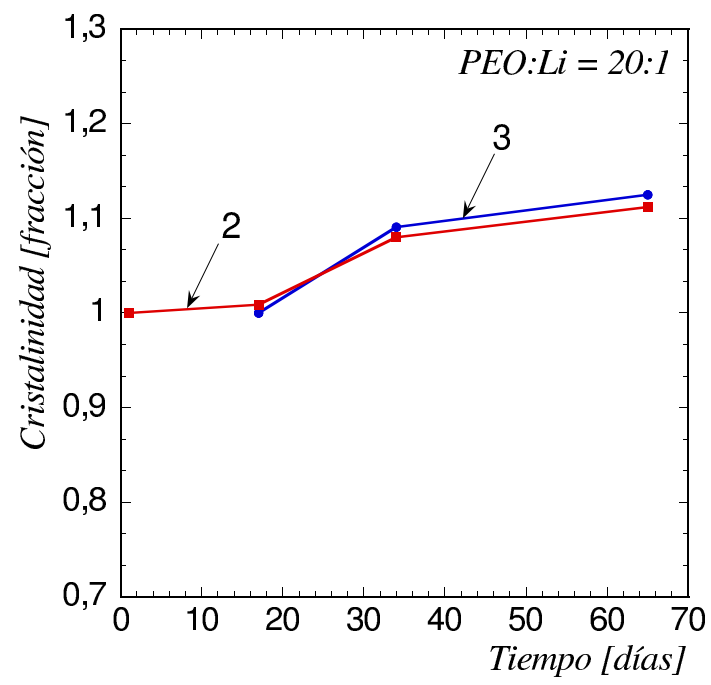

Figura 2.11: Cristalinidad vs. tiempo, películas de concentración PEO:Li=20:1 (probetas 2 y 3). a) Critalinidad porcentual. b) Aumento en relación al día 1.

de litio presente en la muestra. Si vemos la Figura A1 del Apéndice, correspondiente al PEO puro, podemos ver que el evento ocurre con un pico en $68,16{ }^{\circ} \mathrm{C}$ y las probetas con litio oscilan entre los 62,38 y $68,79{ }^{\circ} \mathrm{C}$. Si recordamos que todos los barridos fueron realizados con muestras de una misma probeta, esas variaciones en la posición del pico del evento evidencia que se han tomado partes de la probeta con cierta diferencia en su contenido de litio. Nuevamente aquí nos encontramos con una limitación del método elegido para caracterizar la microestructura desde el punto de vista de su porcentaje de cristalinidad. La técnica de DSC analiza sólo una muy pequeña cantidad de masa del especímen. En una condición ideal de análisis sería esperable tener el pico del evento siempre en el mismo lugar. Otra posibilidad hubiese sido multiplicar la cantidad de barridos de DSC para numerosos trozos de una misma probeta y para cada día de análisis, de modo de poder elegir las curvas de mayor similitud entre sí para correlacionar los datos. El volúmen de trabajo que eso implicaba excedía los recursos disponibles al momento de realizar la campaña experimental, sobre todo por el uso del equipo de calormietría diferencial. Eso no descarta que como trabajo a futuro se pueda pensar en una caracterización de probetas de mayor tamaño, que permitan tomar numerosas muestras para DSC, y generar un muestreo estadístico mayor donde se puedan discriminar mejor las variaciones debidas a la heterogeneidad de la microestructura.

Sin embargo, para poder completar y respaldar los análisis de DSC se decidió realizar las 
a)

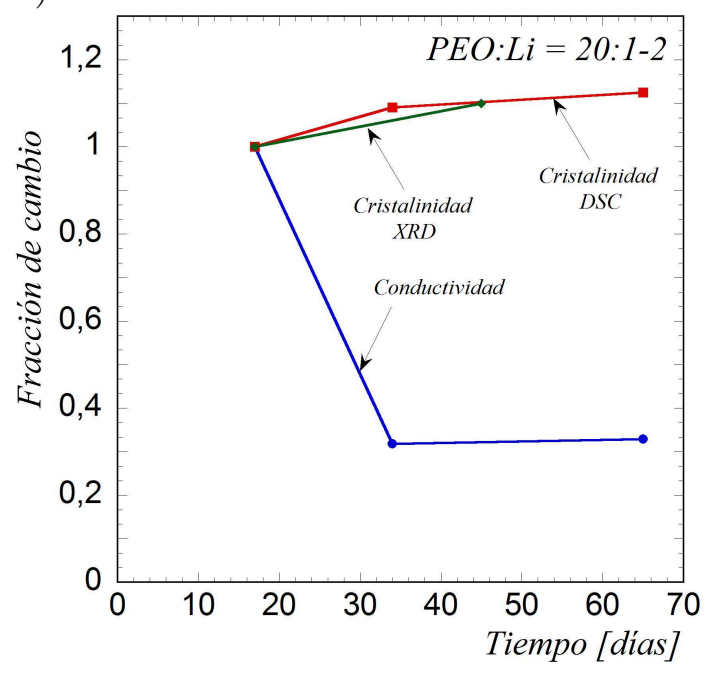

b)

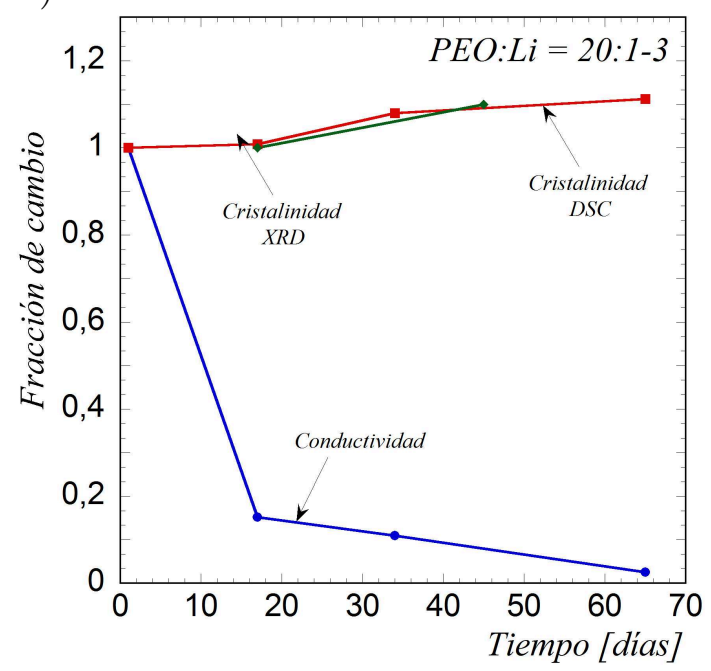

Figura 2.12: Fracción de cambio de la conductividad y la cristalinidad, respecto al valor inicial vs. tiempo para películas de concentración PEO:Li=20:1. a) Probeta 20:1-2 b) Probeta 20:1-3. Obtenidas por DSC y por XRD.

mediciones de Difracción de Rayos X. Al realizar estas pocas corridas se encontró que las variaciones porcentuales de la cristalinidad para períodos de tiempo similares se encuentran dentro de un rango de similitud aceptable, como puede verse en la Figura 2.12. Cabe destacar que como las mediciones de XRD fueron realizadas una vez finalizadas las mediciones de DSC las probetas ensayadas por ambos métodos no son físicamente las mismas. Lo que sí es cierto es que fueron fabricadas de la misma manera, con los mismos materiales y bajo las mismas condiciones y técnicas de laboratorio, con lo cual se tiene un aceptable nivel de confianza de la similitud microestructural. 


\section{Capítulo 3}

\section{Marco Teórico}

En este capítulo se considera el transporte iónico a través de un electrolito sólido microestructurado compuesto por $N$ fases homogéneas diferentes y operando bajo condiciones isotérmicas. Se desprecia cualquier posible efecto debido a deformaciones mecánicas. El electrolito ocupa un dominio fijo $\Omega^{(r)} \subset \Omega(r=1, \ldots, N)$ tal que $\Omega=\cup_{r=1}^{N} \Omega^{(r)}$. Los dominios $\Omega^{(r)}$ pueden ser descriptos por un conjunto de funciones $\chi^{(r)}(\mathbf{x})$, que toman valor igual a 1 si el vector posición $\mathbf{x}$ corresponde a un punto en $\Omega^{(r)}$ o valor 0 en caso contrario. Las fases contienen $A$ especies iónicas con diferentes valencias $z_{\alpha}(\alpha=1, \ldots, A)$. En presencia de un campo eléctrico, dichos iones se mueven a través del medio electrolítico formando una corriente eléctrica. En este trabajo nos concentramos en sistemas electrolíticos donde la longitud de escala de la morfología microestructral es mucho más pequeña que la longitud característica del especímen y de la escala de variación de las solicitaciones del problema. Bajo dichas circunstancias, queda demostrado (véase, por ejemplo, Willis 1983 o Milton 2002) que las propiedades efectivas del electrolito microestructurado son independientes de la forma específica de las acciones externas sobre el sistema. Las derivaciones matemáticas de la siguente sección son particularmente más sencillas cuando la acción externa consiste en un flujo volumétrico de iones hacia el electrolito, el cual es independiente de la microestructura. A continuación, consideremos que $h_{\alpha}(\mathbf{x})$ representa la tasa de producción molar de iones por 
unidad de volumen de las especies $\alpha$ en $\Omega$; el balance de iones requiere que

$$
\dot{c}_{\alpha}+\nabla \cdot \mathbf{j}_{\alpha}=h_{\alpha} \quad \text { en } \Omega, \quad \alpha=1, \ldots, A,
$$

donde $c_{\alpha}(\mathbf{x})$ y $\mathbf{j}_{\alpha}(\mathbf{x})$ indican, respectivamente, la concentración y el flujo de iones del tipo $\alpha$ dentro del electrolito, y el punto superior indica la derivada temporal de dicha cantidad. Siguiendo procedimientos rutinarios, se asocia a cada especie iónica un potencial electroquímico continuo $\mu_{\alpha}(\mathbf{x})$ que caracteriza el flujo de energía debido al transporte de especies. En el marco de este trabajo hemos decidido expresar el flujo de iones como la concentración de los mismos multiplicada por la velocidad de movimiento de dichas cargas, es decir

$$
\mathbf{j}_{\alpha}=c_{\alpha} \mathbf{v}_{\alpha}
$$

con el objetivo de dotar a dicha cantidad de mayor sustento físico observable.

Por ende, asumiendo que los efectos magnéticos son despreciables, el potencial eléctrico $\phi(\mathbf{x})$ en todo el espacio es solución a las ecuaciones de Maxwell

$$
\nabla \cdot \mathbf{d}=F \sum_{\alpha=1}^{A} z_{\alpha} c_{\alpha}, \quad \mathbf{d}=\epsilon_{0} \mathbf{e}+\mathbf{p}, \quad \mathbf{e}=-\nabla \phi \quad \text { en } \mathbb{R}^{3}
$$

donde $F$ es la constante de Faraday, $\epsilon_{0}$ es la permitividad del vacío, $\mathbf{d}(\mathbf{x})$ es el despazamiento eléctrico, $\mathbf{e}(\mathbf{x})$ es la intensidad del campo eléctrico, $\mathbf{p}(\mathbf{x})$ es la polarización del medio electrolítico, y $\phi(\mathbf{x})$ es continuo tal que $\phi \rightarrow 0$ cuando $|\mathbf{x}| \rightarrow \infty$. Finalmente, la densidad de corriente eléctrica $\mathbf{i}(\mathbf{x})$ que fluye por el electrolito queda determinada en función del flujo molar de la siguiente manera

$$
\mathbf{i}=F \sum_{\alpha=1}^{A} z_{\alpha} \mathbf{j}_{\alpha}+\dot{\mathbf{p}}=F \sum_{\alpha=1}^{A} z_{\alpha} c_{\alpha} \mathbf{v}_{\alpha}+\dot{\mathbf{p}} \quad \text { en } \Omega .
$$

Por simplicidad asumimos que las condiciones

$$
\mu_{\alpha}=0 \quad \text { y } \quad \phi=0
$$


se mantienen en el controno $\partial \Omega$. Nótese que la condición de borde en $\phi$ desacopla la distribución del campo eléctrico dentro y fuera de $\Omega$. Además, en vistas de la heterogeneidad del material, las ecuaciones arriba expresadas deben ser entendidas en el sentido de distribuciones, con los campos $c_{\alpha}(\mathbf{x}), \mu_{\alpha}(\mathbf{x}), \phi(\mathbf{x})$ y $\mathbf{p}(\mathbf{x})$ contenidos en $\Omega$.

Las ecuaciones (3.1)-(3.3) deben ser suplementadas por leyes constitutivas apropiadas, consistentes con los principios de la termodinámica ${ }^{1}$. Las mismas son aquí obtenidas siguiendo el procedimiento de Coleman \& Noll (1963). La potencia externa $\mathcal{F}$ debido al flujo externo de iones está dada por

$$
\mathcal{F}=\sum_{\alpha=1}^{A} \int_{\Omega} \mu_{\alpha} h_{\alpha} \mathrm{d} V
$$

donde el producto $\mu_{\alpha} h_{\alpha}$ representa el suministro de energía química asociado a cada especie $\alpha$ (véase, por ejemplo, Gurtin et al. 2010). En virtud del balance 3.1, se puede decir que

$$
\mathcal{F}=\sum_{\alpha=1}^{A} \int_{\Omega} \mu_{\alpha}\left(\dot{c}_{\alpha}+\nabla \cdot \mathbf{j}_{\alpha}\right) \mathrm{d} V
$$

que por teorema de la divergencia puede ser expresado como

$$
\mathcal{F}=\sum_{\alpha=1}^{A} \int_{\Omega}\left(\mu_{\alpha} \dot{c}_{\alpha}-\mathbf{j}_{\alpha} \cdot \nabla \mu_{\alpha}\right) \mathrm{d} V
$$

Por otro lado, la energía libre $\mathcal{E}$ y la tasa de disipación $\mathcal{D}$ del sistema electroquímico son considerados de la siguiente forma

$$
\begin{aligned}
\mathcal{E} & =\int_{\Omega} W\left(\mathbf{x}, \mathbf{p}, c_{\alpha}\right) \mathrm{d} \mathbf{x}+\int_{\Omega} \frac{\epsilon_{0}}{2}|\mathbf{e}|^{2} \mathrm{~d} V \\
\mathcal{D} & =\int_{\Omega} \sum_{\alpha=1}^{A} c_{\alpha} \frac{\partial U^{*}}{\partial \mathbf{v}_{\alpha}}\left(\mathbf{x}, \mathbf{v}_{\alpha}\right) \cdot \mathbf{v}_{\alpha} \mathrm{d} V
\end{aligned}
$$

En la expresión (3.9) el primer término representa la energía del electrolito mientras que el segundo término representa la energía del campo eléctrico; en la expresión (3.10) el integrando representa la tasa de la energía por unidad de volumen disipada en el electrolito debido al

\footnotetext{
${ }^{1}$ Por otra parte, las condiciones iniciales no serán requeridas en los análisis subsiguientes.
} 
transporte iónico. La posible disipación debido a la polarizabilidad eléctrica es despreciada. Siguiendo pasos habituales en estos tipos de análisis (ej., Germain et al. 1983), se asume que la densidad de energía $W$ y el potencial de disipación $U$ son convexos en las variables $c_{\alpha}, \mathbf{p}$ y $\mathbf{v}_{\alpha}$. Además, se asume que $U$ es positivo y que toma el valor 0 en $c_{\alpha}=0$ y $\mathbf{v}_{\alpha}=0$. Estas propiedades garantizan que la disipación sea positiva, como es requerido por las leyes de la termodinámica.

Ahora bien, la tasa de disipación (3.10) debe ser igual a la diferencia entre la tasa del trabajo externo (3.6) y la tasa de cambio de la energía libre (3.9) para todos los procesos termodinámicamente admisibles. Es decir

$$
\mathcal{D}=\mathcal{F}-\frac{d \mathcal{E}}{d t}
$$

Veremos ahora el desarrollo matemático para obtener la derivada temporal de la energía libre, para hacerlo nos hemos basado en el trabajo de Xiao \& Bhattacharya (2008). Haciendo uso de (3.9) en (3.11) tenemos que

$$
\frac{d \mathcal{E}}{d t}=\frac{d}{d t}\left[\int_{\Omega} W \mathrm{~d} \mathbf{x}+\int_{\Omega} \frac{\epsilon_{0}}{2}|\mathbf{e}|^{2} \mathrm{~d} V\right]
$$

Veamos el cálculo del primer término del lado derecho del igual de la ecuación (3.12):

$$
\frac{d}{d t}\left[\int_{\Omega} W \mathrm{~d} \mathbf{x}\right]=\int_{\Omega} \dot{W}\left(\mathbf{x}, \mathbf{p}, c_{\alpha}\right) \mathrm{d} \mathbf{x}=\int_{\Omega}\left(\frac{\partial W}{\partial \mathbf{p}} \cdot \dot{\mathbf{p}}+\frac{\partial W}{\partial c_{\alpha}} \cdot \dot{c_{\alpha}}\right) \mathrm{d} \mathbf{x} .
$$

Ahora veremos la derivada respecto del tiempo del segundo término de (3.12):

$$
\frac{d}{d t}\left[\int_{\Omega} \frac{\epsilon_{0}}{2}|\mathbf{e}|^{2} \mathrm{~d} V\right]=\frac{d}{d t}\left[\int_{\Omega} \frac{\epsilon_{0}}{2}|\nabla \phi|^{2} \mathrm{~d} V\right]
$$

Utilizaremos las ecuaciones de Maxwell (3.3) sabiendo que

$$
\nabla \cdot \mathbf{d}-F \sum_{\alpha=1}^{A} z_{\alpha} c_{\alpha}=0
$$

Multiplicándola por un campo potencial genérico $\psi(\mathbf{x})$ y expresándola en su forma integral, 
se puede decir que

$$
\int_{\Omega}\left(\nabla \cdot \mathbf{d}-F \sum_{\alpha=1}^{A} z_{\alpha} c_{\alpha}\right) \psi(\mathbf{x}) \mathrm{d} \mathbf{x}=0 \quad \forall \psi(\mathbf{x})
$$

con lo cual

$$
\int_{\Omega} \nabla \cdot\left(-\epsilon_{0} \nabla \phi+\mathbf{p}\right) \psi(\mathbf{x}) \mathrm{d} \mathbf{x}=\int_{\Omega}\left(F \sum_{\alpha=1}^{A} z_{\alpha} c_{\alpha}\right) \psi(\mathbf{x}) \mathrm{d} \mathbf{x} .
$$

Como la expresión anterior es válida para cualquier campo $\psi(\mathbf{x})$, particularmente para $\psi(\mathbf{x})=\phi(\mathbf{x})$, y aplicando el teorema de la divergencia, tenemos que

$$
\int_{\Omega} \nabla \cdot\left(-\epsilon_{0} \nabla \phi+\mathbf{p}\right) \phi(\mathbf{x}) \mathrm{d} \mathbf{x}=-\int_{\Omega}\left(-\epsilon_{0} \nabla \phi+\mathbf{p}\right) \cdot \nabla \phi(\mathbf{x}) \mathrm{d} \mathbf{x}
$$

con lo cual

$$
-\int_{\Omega}\left(-\epsilon_{0} \nabla \phi+\mathbf{p}\right) \cdot \nabla \phi(\mathbf{x}) \mathrm{d} \mathbf{x}=F \int_{\Omega}\left(\sum_{\alpha=1}^{A} z_{\alpha} c_{\alpha}\right) \phi(\mathbf{x}) \mathrm{d} \mathbf{x} .
$$

Luego

$$
\int_{\Omega} \epsilon_{0} \nabla \phi \cdot \nabla \phi \mathrm{d} \mathbf{x}=\int_{\Omega} \mathbf{p} \cdot \nabla \phi(\mathbf{x}) \mathrm{d} \mathbf{x}+F \int_{\Omega}\left(\sum_{\alpha=1}^{A} z_{\alpha} c_{\alpha}\right) \phi(\mathbf{x}) \mathrm{d} \mathbf{x}
$$

es decir que

$$
\frac{d}{d t}\left[\int_{\Omega} \epsilon_{0}|\nabla \phi|^{2} \mathrm{~d} V\right]=\frac{d}{d t}\left[\int_{\Omega} \mathbf{p} \cdot \nabla \phi(\mathbf{x}) \mathrm{d} \mathbf{x}+F \int_{\Omega}\left(\sum_{\alpha=1}^{A} z_{\alpha} c_{\alpha}\right) \phi(\mathbf{x}) \mathrm{d} \mathbf{x}\right] .
$$

Derivando los productos dentro de las integrales se obtiene que el lado derecho de (3.21) es

$$
\begin{aligned}
& \frac{d}{d t}\left[\int_{\Omega} \epsilon_{0}|\mathbf{e}|^{2} \mathrm{~d} V\right]=\int_{\Omega}(\nabla \dot{\phi}(\mathbf{x}) \cdot \mathbf{p}+\nabla \phi(\mathbf{x}) \cdot \dot{\mathbf{p}}) \mathrm{d} \mathbf{x} \\
& +F \int_{\Omega}\left(\sum_{\alpha=1}^{A} z_{\alpha} \dot{c}_{\alpha} \phi(\mathbf{x})+\sum_{\alpha=1}^{A} z_{\alpha} c_{\alpha} \dot{\phi}(\mathbf{x})\right) \mathrm{d} \mathbf{x} .
\end{aligned}
$$


Volvamos ahora a la ecuación (3.16), aplicando el mismo concepto pero ahora multiplicando ambos términos por un campo potencial $\psi(\mathbf{x})=\dot{\phi}(\mathbf{x})$

$$
\int_{\Omega}\left(\nabla \cdot \mathbf{d}-F \sum_{\alpha=1}^{A} z_{\alpha} c_{\alpha}\right) \dot{\phi}(\mathbf{x}) \mathrm{d} \mathbf{x}=0 \quad \forall \dot{\phi}(\mathbf{x})
$$

Nuevamente, por teorema de la divergencia, se puede decir que

$$
\int_{\Omega} \nabla \cdot\left(-\epsilon_{0} \nabla \phi+\mathbf{p}\right) \dot{\phi}(\mathbf{x}) \mathrm{d} \mathbf{x}=\int_{\Omega} \epsilon_{0} \nabla \dot{\phi} \cdot \nabla \phi \mathrm{d} \mathbf{x}-\int_{\Omega} \nabla \dot{\phi} \cdot \mathbf{p} \mathrm{d} \mathbf{x}
$$

reemplazando en (3.23) se tiene que

$$
\int_{\Omega} \epsilon_{0} \nabla \phi \cdot \nabla \dot{\phi} \mathrm{d} \mathbf{x}=\int_{\Omega} \nabla \dot{\phi} \cdot \mathbf{p} \mathrm{d} \mathbf{x}+F \sum_{\alpha=1}^{A} z_{\alpha} c_{\alpha} \dot{\phi}(\mathbf{x}) \mathrm{d} \mathbf{x}
$$

Luego,

$$
\frac{d}{d t}\left[\frac{1}{2} \int_{\Omega} \epsilon_{0}|\nabla \phi|^{2} \mathrm{~d} \mathbf{x}\right]=\frac{\epsilon_{0}}{2} \int_{\Omega} \frac{\partial}{\partial t}|\nabla \phi|^{2} \mathrm{~d} \mathbf{x}=\epsilon_{0} \int_{\Omega} \nabla \phi \cdot \nabla \dot{\phi} \mathrm{d} \mathbf{x} .
$$

Finalmente, tomando la expresión (3.22) y restándole (3.26)

$$
\begin{aligned}
& \frac{d}{d t}\left[\int_{\Omega} \epsilon_{0}|\nabla \phi|^{2} \mathrm{~d} V-\frac{1}{2} \int_{\Omega} \epsilon_{0}|\nabla \phi|^{2} \mathrm{~d} \mathbf{x}\right]=\int_{\Omega}(\nabla \dot{\phi} \cdot \mathbf{p}+\nabla \phi \cdot \dot{\mathbf{p}}) \mathrm{d} \mathbf{x} \\
& +F \int_{\Omega}\left(\sum_{\alpha=1}^{A} z_{\alpha} \dot{c}_{\alpha} \phi+\sum_{\alpha=1}^{A} z_{\alpha} c_{\alpha} \dot{\phi}\right) \mathrm{d} \mathbf{x}-\left[\int_{\Omega}\left(\nabla \dot{\phi} \cdot \mathbf{p} \mathrm{d} \mathbf{x}+F \sum_{\alpha=1}^{A} z_{\alpha} c_{\alpha}\right) \dot{\phi} \mathrm{d} \mathbf{x}\right]
\end{aligned}
$$

Con lo cual se llega a que

$$
\frac{d}{d t}\left[\frac{\epsilon_{0}}{2} \int_{\Omega}|\nabla \phi|^{2} \mathrm{~d} \mathbf{x}\right]=\int_{\Omega} \nabla \phi \cdot \dot{\mathbf{p}} \mathrm{d} \mathbf{x}+F \int_{\Omega} \sum_{\alpha=1}^{A} z_{\alpha} \dot{c}_{\alpha} \phi \mathrm{d} \mathbf{x} .
$$

Volvamos ahora a la ecuación (3.11), reemplazando en ella las expresiones (3.8), (3.10), 
(3.13) y (3.28) obtenemos

$$
\begin{aligned}
& \int_{\Omega} \sum_{\alpha=1}^{A} c_{\alpha} \frac{\partial U}{\partial \mathbf{v}_{\alpha}} \cdot \mathbf{v}_{\alpha} \mathrm{d} V=\sum_{\alpha=1}^{A} \int_{\Omega}\left(\mu_{\alpha} \dot{c}_{\alpha}-\mathbf{j}_{\alpha} \cdot \nabla \mu_{\alpha}\right) \mathrm{d} V+\int_{\Omega}\left(\frac{\partial W}{\partial \mathbf{p}} \cdot \dot{\mathbf{p}}-\frac{\partial W}{\partial c_{\alpha}} \cdot \dot{c_{\alpha}}\right) \mathrm{d} \mathbf{x} \\
& -\int_{\Omega} \nabla \phi \cdot \dot{\mathbf{p}} \mathrm{d} \mathbf{x}-F \int_{\Omega}\left(\sum_{\alpha=1}^{A} z_{\alpha} \dot{c_{\alpha}}\right) \phi \mathrm{d} \mathbf{x} .
\end{aligned}
$$

Esto implica que

$$
\begin{aligned}
& \int_{\Omega} \sum_{\alpha=1}^{A}\left(\mu_{\alpha}-\frac{\partial W}{\partial c_{\alpha}}-F z_{\alpha} \phi\right) \cdot \dot{c}_{\alpha} \mathrm{d} V+\int_{\Omega}\left(\mathbf{e}-\frac{\partial W}{\partial \mathbf{p}}\right) \cdot \dot{\mathbf{p}} \mathrm{d} V \\
- & \int_{\Omega} \sum_{\alpha=1}^{A} c_{\alpha}\left(\nabla \mu_{\alpha}+\frac{\partial U}{\partial \mathbf{v}_{\alpha}}\right) \cdot \mathrm{v}_{\alpha} \mathrm{d} V=0
\end{aligned}
$$

debe cumplirse para todos los procesos termodinámicamente admisibles.

De los procedimientos anteriores se obtienen las relaciones constitutivas del problema de transporte iónico, las cuales caracterizan la naturaleza del mismo. En el marco de esta tesis, dicho problema físico ha sido resuelto tanto en su versión lineal, como se verá en el Capítulo 4, como en su manera más general para casos no lineales, como se detalla en el Capítulo 7. Por lo pronto se detallan las ecuaciones de manera general, lo cual implica las siguientes relaciones constitutivas

$$
\mathbf{e}=\frac{\partial W}{\partial \mathbf{p}}, \quad \mu_{\alpha}=\frac{\partial W}{\partial c_{\alpha}}+F z_{\alpha} \phi, \quad \nabla \mu_{\alpha}=-\frac{\partial U^{*}}{\partial \mathbf{v}_{\alpha}}
$$

Avanzar en el análisis requiere formas específicas de las funciones $W$ y $U$, dichas funciones pueden ser escritas de la siguiente manera

$$
\begin{aligned}
W\left(\mathbf{x}, \mathbf{p}, c_{\alpha}\right) & =\sum_{r=1}^{N} \chi^{(r)}(\mathbf{x}) W^{(r)}\left(\mathbf{p}, c_{\alpha}\right), \\
U^{*}\left(\mathbf{x}, \mathbf{v}_{\alpha}\right) & =\sum_{r=1}^{N} \chi^{(r)}(\mathbf{x}) U^{*(r)}\left(\mathbf{v}_{\alpha}\right)
\end{aligned}
$$

donde $W^{(r)}$ y $U^{(r)}$ indican las densidades de energía y los potenciales de disipación aso- 
ciados con cada fase $r$. Se asume que las funciones $W^{(r)}(\cdot, \cdot)$ y $U^{(r)}(\cdot)$ son convexas. Adicionalmente, las funciones $U^{(r)}$ son asumidas positivas y que toman valor 0 en $c_{\alpha}=0 \mathrm{y} \mathbf{v}_{\alpha}=0$. Dichas propiedades garantizan la disipación positiva, como es requerido por las leyes de la termodinámica.

En este trabajo, se asumen formas más específicas

$$
\begin{aligned}
W^{(r)}\left(\mathbf{p}, c_{\alpha}\right) & =W_{p}^{(r)}(\mathbf{p})+R T \sum_{\alpha=1}^{A} c_{\alpha}\left(\ln \frac{c_{\alpha}}{c_{0 \alpha}^{(r)}}-1\right) \\
U^{*(r)}\left(\mathbf{v}_{\alpha}\right) & =\sum_{\alpha=1}^{A} U_{\alpha}^{*(r)}\left(\mathbf{v}_{\alpha}\right) .
\end{aligned}
$$

Aquí, $W_{p}^{(r)}$ es la energía interna acumulada en la fase $r$ por la polarizabilidad eléctrica, $R T$ es la constante universal de los gases multiplicada por la temperatura absoluta, $c_{0 \alpha}^{(r)}$ es una concentración molar de referencia característica de la fase $r$, y $U^{(r)}$ es un potencial de disipación asociado con la movilidad iónica a través de la fase $r$. Introduciendo dichas funciones en (3.31) se obtienen las siguientes relaciones constitutivas consistentes con las leyes de la termodinámica:

$$
\mathbf{e}=\frac{\partial W_{p}^{(r)}}{\partial \mathbf{p}}(\mathbf{p}), \quad \mu_{\alpha}=R T \ln \frac{c_{\alpha}}{c_{0 \alpha}^{(r)}}+F z_{\alpha} \phi, \quad \nabla \mu_{\alpha}=-\frac{\partial U_{\alpha}^{*(r)}}{\partial \mathbf{v}_{\alpha}}\left(\mathbf{v}_{\alpha}\right)
$$

Estas relaciones consitutivas son apropiadas para electrolitos sólidos que contengan concentraciones diluidas de iones. Cabe tener en cuenta que cuando las concentraciones de referencia $c_{0}^{(r)}$ difieren entre las fases, las concentraciones molares $c_{\alpha}$ son discontinuas en las interfaces materiales internas. De hecho, la relación $c_{0 \alpha}^{(r)} / c_{0 \alpha}^{(s)}$ fija la relación de concentraciones molares de las especies iónicas $\alpha$ en las fases $r$ y $s$ en condiciones de equilibrio.

Nótese que en la relación constitutiva $\left(3.36_{3}\right)$, la función $U_{\alpha}^{*(r)}$ es invertible tal que

$$
\mathbf{v}_{\alpha}=-\frac{\partial U_{\alpha}^{(r)}}{\partial \nabla \mu_{\alpha}}\left(\nabla \mu_{\alpha}\right)
$$


y por lo tanto se puede expresar el flujo molar de iones como

$$
\mathbf{j}_{\alpha}=-c_{\alpha} \frac{\partial U_{\alpha}^{(r)}}{\partial \nabla \mu_{\alpha}}\left(\nabla \mu_{\alpha}\right)
$$

Como veremos más adelante cuando se trabaje sobre el problema lineal se utilizarán potenciales de disipación $U_{\alpha}^{(r)}$ cuadráticos que arrojen relaciones lineales. Por lo contrario cuando se esté trabajando en el ámbito no lineal, la suposición fundamental en dicho análisis será que la alinealidad de la respuesta efectiva bajo ciertas intensidades de campo eléctrico es de hecho manifestación de la dependencia no lineal del flujo molar de iones con los gradientes del potencial electroquímico de las especies de iones dentro de sus fases constitutivas. Sus descripciones requerirán por ende el uso de potenciales de disipación $U_{\alpha}^{(r)}$ supracuadráticos. 


\section{Capítulo 4}

\section{Respuesta Efectiva}

\subsection{Transporte iónico lineal}

En este capítulo detallaremos los procedimientos teóricos para arribar a la obtención de las propiedades efectivas de un electrolito sólido cuyas fases presentan comportamientos lineales. Como se vio en el Capítulo 3 es necesario especificar la forma que tienen las relaciones constitutivas a fin de poder avanzar con la resolución del problema. En este trabajo se caracteriza la respuesta electroquímica de las fases constitutivas por funciones de la siguiente forma

$$
\begin{aligned}
W^{(r)}\left(\mathbf{p}, c_{1}, \ldots, c_{A}\right) & =\frac{1}{2} \mathbf{p} \cdot \boldsymbol{\kappa}^{(r)} \mathbf{p}+R T \sum_{\alpha=1}^{A} c_{\alpha}\left(\ln \frac{c_{\alpha}}{c_{0 \alpha}^{(r)}}-1\right) \\
U^{(r)}\left(\nabla \mu_{1}, \ldots, \nabla \mu_{A}\right) & =\sum_{\alpha=1}^{A} \frac{1}{2} \nabla \mu_{\alpha} \cdot \mathbf{M}_{\alpha}^{(r)} \nabla \mu_{\alpha} .
\end{aligned}
$$

Donde $\boldsymbol{\kappa}^{(r)}$ y $\mathbf{M}_{\alpha}^{(r)}$ son tensores de segundo orden, positivos definidos, que caracterizan la polarizabilidad eléctrica y la movilidad iónica de cada fase $r$, respectivamente. Introduciendo las expresiones en (3.31) se obtienen las siguientes relaciones consitutivas, consistentes 
termodinámicamente:

$$
\mathbf{p}=\left(\boldsymbol{\kappa}^{(r)}\right)^{-1} \mathbf{e}, \quad \mu_{\alpha}=R T \ln \frac{c_{\alpha}}{c_{0 \alpha}^{(r)}}+F z_{\alpha} \phi, \quad \mathbf{j}_{\alpha}=-c_{\alpha} \mathbf{M}_{\alpha}^{(r)} \nabla \mu_{\alpha}
$$

Los desarrollos subsiguientes se ven simplificados si se introducen las siguientes expresiones para los tensores de permitividad eléctrica y de pseudo-difusividad

$$
\boldsymbol{\epsilon}^{(r)}=\epsilon_{0} \mathbf{I}+\left(\boldsymbol{\kappa}^{(r)}\right)^{-1}, \quad \mathbb{D}_{\alpha}^{(r)}\left(\mu_{\alpha}, \phi\right)=g\left(\mu_{\alpha}-F z_{\alpha} \phi\right) c_{0 \alpha}^{(r)} \mathbf{M}_{\alpha}^{(r)}
$$

donde

$$
g(s)=e^{s / R T}
$$

La función exponencial en estas expresiones aparece por la eliminación de las concentraciones molares $c_{\alpha}$ invirtiendo la expresión $(4.3)_{2}$. En vista de estas definiciones, las relaciones constitutivas para el flujo molar y el desplazamiento eléctrico dentro de cada fase toman una forma sencilla

$$
\mathbf{j}_{\alpha}=-\mathbb{D}_{\alpha}^{(r)}\left(\mu_{\alpha}, \phi\right) \nabla \mu_{\alpha} \quad \mathrm{y} \quad \mathbf{d}=\boldsymbol{\epsilon}^{(r)} \mathbf{e}
$$

Nótese que la difusividad de las diferentes familias iónicas están sólo acopladas a través del potencial eléctrico $\phi$.

\subsection{Respuesta efectiva}

\subsubsection{Problema con múltiples escalas}

La respuesta efectiva del electrolito microestructurado es formalmente obtenida evaluando el transporte de iones a través de una secuencia de sistemas materiales con $\Omega$ fijo y con longitudes de escala más pequeñas y decrecientes. Hay varias maneras de evaluar dicho límite. En este trabajo hemos hecho uso del método de convergencia multi-escala con el enfoque 
propuesto por Allaire \& Briane (1996). Este método asume que la microestructura es una repetición periódica de una unidad elemental cúbica con posibles escalas de longitud múltiples. Dado nuestro interés en sistemas de polímeros semi-cristalinos, consideramos sistemas materiales que poseen dos escalas de longitud microestructurales bien separadas, que luego serán identificadas con las longitudes características del laminado y de las esferulitas. Más específicamente, $\Omega_{\#}=[0, \ell]^{3}$ se refiere a una unidad elemental cúbica de lado $\ell$ y se escriben las funciones características $\chi^{(r)}$ como el producto

$$
\chi^{(r)}(\mathbf{x})=\chi_{1}^{(r)}\left(\frac{\mathbf{x}}{\varepsilon}\right) \chi_{2}^{(r)}\left(\frac{\mathbf{x}}{\varepsilon^{2}}\right)
$$

donde $\varepsilon=\left|\Omega_{\#}\right| /|\Omega|$; además las funciones $\chi_{1}^{(r)}$ y $\chi_{2}^{(r)}$ son periódicas en $\Omega_{\#} \mathrm{y}$ son las funciones características que describen los patrones morfológicos tanto en la mayor como la menor escala de longitud, respectivamente. En esta descripción microestructural basada en las fases, una fase constitutiva específica puede ser nombrada como dos materiales diferentes en distintos patrones de mesoescala; alternativamente, microestructuras multi-escala pueden ser descriptas por funciones características basadas en patrones morfológicos como en Agoras \& Ponte Castañeda (2011).

En vista de las expresiones (3.1) y (4.6), las correspondientes secuencias de potenciales electroquímicos $\mu_{\alpha}^{\varepsilon}$ y de campos eléctricos $\phi^{\varepsilon}$ en un régimen estacionario son solución de las ecuaciones no lineales de campo

$$
\begin{array}{cr}
-\nabla \cdot\left[\mathbb{D}_{\alpha}\left(\frac{\mathbf{x}}{\varepsilon}, \frac{\mathbf{x}}{\varepsilon^{2}}, \mu_{\alpha}^{\varepsilon}, \phi^{\varepsilon}\right) \nabla \mu_{\alpha}^{\varepsilon}\right]=h_{\alpha}(\mathbf{x}) & \text { en } \Omega, \\
-\nabla \cdot\left[\epsilon\left(\frac{\mathbf{x}}{\varepsilon}, \frac{\mathbf{x}}{\varepsilon^{2}}\right) \nabla \phi^{\varepsilon}\right]=F \sum_{\alpha=1}^{A} z_{\alpha} c_{0 \alpha}\left(\frac{\mathbf{x}}{\varepsilon}, \frac{\mathbf{x}}{\varepsilon^{2}}\right) g\left(\mu_{\alpha}^{\varepsilon}-F z_{\alpha} \phi^{\varepsilon}\right) & \text { en } \Omega, \\
\mu_{\alpha}^{\varepsilon}=0 \quad \text { y } \quad \phi^{\varepsilon}=0 & \text { en } \partial \Omega,
\end{array}
$$

donde 


$$
\begin{aligned}
\mathbb{D}_{\alpha}\left(\mathbf{y}, \mathbf{z}, \mu_{\alpha}, \phi\right) & =\sum_{r=1}^{N} \chi_{1}^{(r)}(\mathbf{y}) \chi_{2}^{(r)}(\mathbf{z}) \mathbb{D}_{\alpha}^{(r)}\left(\mu_{\alpha}, \phi\right), \\
\epsilon(\mathbf{y}, \mathbf{z}) & =\sum_{r=1}^{N} \chi_{1}^{(r)}(\mathbf{y}) \chi_{2}^{(r)}(\mathbf{z}) \boldsymbol{\epsilon}^{(r)} \\
c_{0 \alpha}(\mathbf{y}, \mathbf{z}) & =\sum_{r=1}^{N} \chi_{1}^{(r)}(\mathbf{y}) \chi_{2}^{(r)}(\mathbf{z}) c_{0 \alpha}^{(r)}
\end{aligned}
$$

con $\alpha=1, \ldots, A$. La respuesta homogenizada del sistema es obtenida tomando el límite $\varepsilon \rightarrow 0$ en estas expresiones.

\subsubsection{Limites}

Se asumen que los campos $\mu_{\alpha}^{\varepsilon}(\mathbf{x})$ y $\phi^{\varepsilon}(\mathbf{x})$, que son solución de las ecuaciones (4.8)(4.9), están acotados en $H_{0}^{1}(\Omega)$ para todo $\varepsilon$. Luego, hasta una subsecuencia, dichos campos multi-escala covergen a ciertos campos límite $\bar{\mu}_{\alpha}(\mathbf{x})$ y $\bar{\phi}(\mathbf{x})$ en $H_{0}^{1}(\Omega)$ respectivamente, mientras que sus gradientes multi-escala convergen a ciertos campos gradientes límite $\nabla \bar{\mu}_{\alpha}(\mathbf{x})+\nabla_{y} \mu_{\alpha}^{1}(\mathbf{x}, \mathbf{y})+\nabla_{z} \mu_{\alpha}^{2}(\mathbf{x}, \mathbf{y}, \mathbf{z})$ y $\nabla \bar{\phi}(\mathbf{x})+\nabla_{y} \phi^{1}(\mathbf{x}, \mathbf{y})+\nabla_{z} \phi^{2}(\mathbf{x}, \mathbf{y}, \mathbf{z})$ respectivamente siendo las funciones $\mu_{\alpha}^{i} \mathrm{y} \phi^{i}(\mathbf{x}, \cdot)$ periódicas en $\Omega_{\#}$ en los argumentos $\mathbf{y}$ y $\mathbf{z}$ —véase Allaire \& Briane (1996). Las ecuaciones para las diferentes funciones límite se obtienen escribiendo las ecuaciones de campo (4.8)-(4.9) en su forma débil y pasando al límite $\varepsilon \rightarrow 0$.

Ecuaciones de difusión. Para una dada secuencia de potenciales eléctricos $\phi^{\varepsilon}$, las ecuaciones (4.8) para el potencial electroquímico $\mu_{\alpha}^{\varepsilon}$ pueden ser escritas en la forma débil de la siguiente manera

$$
\int_{\Omega}\left[\nabla \cdot \mathbb{D}_{\alpha}\left(\frac{\mathbf{x}}{\varepsilon}, \frac{\mathbf{x}}{\varepsilon^{2}}, \mu_{\alpha}^{\varepsilon}, \phi^{\varepsilon}\right) \nabla \mu_{\alpha}^{\varepsilon}+h_{\alpha}(\mathbf{x})\right] \varphi(\mathbf{x}) \mathrm{d} V=0 \quad \forall \varphi \in H_{0}^{1}(\Omega) .
$$

Tomando $\varphi(\mathbf{x})=\varphi_{0}(\mathbf{x})+\varepsilon \varphi_{1}(\mathbf{x}, \mathbf{x} / \varepsilon)+\varepsilon^{2} \varphi_{2}\left(\mathbf{x}, \mathbf{x} / \varepsilon, \mathbf{x} / \varepsilon^{2}\right)$, con $\varphi_{i}(\mathbf{x}, \cdot)$ periódica en $\Omega_{\#}$ en 
su segundo y tercer argumento, e integrando por partes se obtiene que

$$
\begin{array}{r}
\lim _{\varepsilon \rightarrow 0} \int_{\Omega} \nabla \mu_{\alpha}^{\varepsilon} \cdot \mathbb{D}_{\alpha}\left(\frac{\mathbf{x}}{\varepsilon}, \frac{\mathbf{x}}{\varepsilon^{2}}, \mu_{\alpha}^{\varepsilon}, \phi^{\varepsilon}\right)\left[\nabla \varphi_{0}(\mathbf{x})+\nabla_{y} \varphi_{1}(\mathbf{x}, \mathbf{x} / \varepsilon)+\nabla_{z} \varphi_{2}\left(\mathbf{x}, \mathbf{x} / \varepsilon, \mathbf{x} / \varepsilon^{2}\right)\right] \mathrm{d} V \\
=\int_{\Omega} h_{\alpha}(\mathbf{x}) \varphi_{0}(\mathbf{x}) \mathrm{d} V
\end{array}
$$

para todas las funciones $\varphi_{0}, \varphi_{1}$ y $\varphi_{2}$ admisibles. Basándose en el trabajo de Casado-Díaz \& Gayte (2002) sobre operadores cuasi-lineales que oscilan en una sola escala de longitud y satisfacen ciertas condiciones de crecimiento polinomial, es razonable esperar que la igualdad

$$
\begin{aligned}
& \lim _{\varepsilon \rightarrow 0} \int_{\Omega} \nabla \mu_{\alpha}^{\varepsilon} \cdot \mathbb{D}_{\alpha}\left(\frac{\mathbf{x}}{\varepsilon}, \frac{\mathbf{x}}{\varepsilon^{2}}, \mu_{\alpha}^{\varepsilon}, \phi^{\varepsilon}\right)\left[\nabla \varphi_{0}(\mathbf{x})+\nabla_{y} \varphi_{1}(\mathbf{x}, \mathbf{x} / \varepsilon)+\nabla_{z} \varphi_{2}\left(\mathbf{x}, \mathbf{x} / \varepsilon, \mathbf{x} / \varepsilon^{2}\right)\right] \mathrm{d} V= \\
& \lim _{\varepsilon \rightarrow 0} \int_{\Omega} \nabla \mu_{\alpha}^{\varepsilon} \cdot \mathbb{D}_{\alpha}\left(\frac{\mathbf{x}}{\varepsilon}, \frac{\mathbf{x}}{\varepsilon^{2}}, \bar{\mu}_{\alpha}, \bar{\phi}\right)\left[\nabla \varphi_{0}(\mathbf{x})+\nabla_{y} \varphi_{1}(\mathbf{x}, \mathbf{x} / \varepsilon)+\nabla_{z} \varphi_{2}\left(\mathbf{x}, \mathbf{x} / \varepsilon, \mathbf{x} / \varepsilon^{2}\right)\right] \mathrm{d} V
\end{aligned}
$$

se mantenga válida ${ }^{1}$. Asumida esta igualdad, la ecuación (4.14) y la definición de convergencia multi-escala implican que

$$
\begin{array}{r}
\int_{\Omega}\left\langle\left[\nabla \bar{\mu}_{\alpha}(\mathbf{x})+\nabla_{y} \mu_{\alpha}^{1}(\mathbf{x}, \mathbf{y})+\nabla_{z} \mu_{\alpha}^{2}(\mathbf{x}, \mathbf{y}, \mathbf{z})\right] \cdot \mathbb{D}_{\alpha}\left(\mathbf{y}, \mathbf{z}, \bar{\mu}_{\alpha}, \bar{\phi}\right)\right. \\
\left.\left[\nabla \varphi_{0}(\mathbf{x})+\nabla_{y} \varphi_{1}(\mathbf{x}, \mathbf{y})+\nabla_{z} \varphi_{2}(\mathbf{x}, \mathbf{y}, \mathbf{z})\right]\right\rangle_{y, z} \mathrm{~d} V=\int_{\Omega} h_{\alpha}(\mathbf{x}) \varphi_{0}(\mathbf{x}) \mathrm{d} V
\end{array}
$$

para todas las funciones $\varphi_{0}, \varphi_{1}$ y $\varphi_{2}$ admisibles, donde $\nabla_{y}$ y $\nabla_{z}$ se refieren al operador nabla parcial con respecto a y y z, respectivamente, $\langle\cdot\rangle_{y},\langle\cdot\rangle_{z} \mathrm{y}\langle\cdot\rangle_{y, z}$ denotan los promedios volumétricos sobre $\mathbf{y}$, sobre $\mathbf{z}$, y simultáneamente sobre y y z, respectivamente. Finalmente, variaciones respecto a $\varphi_{0}, \varphi_{1}$ y $\varphi_{2}$ conducen a las ecuaciones de campo (4.26) y (4.30).

Ecuaciones de Gauss. Recalcando que la función $g$ es la derivada de una función convexa $f$, es sencillo demostrar que, para campos $\mu_{\alpha}^{\varepsilon}$ dados, el potencial eléctrico $\phi^{\varepsilon}$ que satisface (4.9)

\footnotetext{
${ }^{1}$ Desafortunadamente una demostración rigurosa no esta disponible aún.
} 
resuelve el problema de minimización

$$
\bar{I}^{\varepsilon}=\min _{\phi^{\varepsilon} \in H_{0}^{1}(\Omega)} I^{\varepsilon}\left[\phi^{\varepsilon}\right]=\min _{\phi^{\varepsilon} \in H_{0}^{1}(\Omega)} \int_{\Omega}\left[\frac{1}{2} \nabla \phi^{\varepsilon} \cdot \boldsymbol{\epsilon}\left(\frac{\mathbf{x}}{\varepsilon}, \frac{\mathbf{x}}{\varepsilon^{2}}\right) \nabla \phi^{\varepsilon}+\sum_{\alpha=1}^{A} c_{0 \alpha}\left(\frac{\mathbf{x}}{\varepsilon}, \frac{\mathbf{x}}{\varepsilon^{2}}\right) f\left(\mu_{\alpha}^{\varepsilon}-F z_{\alpha} \phi^{\varepsilon}\right)\right] \mathrm{d} V .
$$

El funcional $I^{\varepsilon}\left[\phi^{\varepsilon}\right]$ puede ser acotado tanto por encima como por debajo. Por otra parte, la convexidad del integrando de (4.18) en $\nabla \phi^{\varepsilon}$ y $\phi^{\varepsilon}$ implica que

$$
\begin{array}{r}
I^{\varepsilon}\left[\phi^{\varepsilon}\right] \geq \int_{\Omega}\left[\frac{1}{2} \mathbf{e} \cdot \boldsymbol{\epsilon}\left(\frac{\mathbf{x}}{\varepsilon}, \frac{\mathbf{x}}{\varepsilon^{2}}\right) \mathbf{e}+\boldsymbol{\epsilon}\left(\frac{\mathbf{x}}{\varepsilon}, \frac{\mathbf{x}}{\varepsilon^{2}}\right) \mathbf{e} \cdot\left(\nabla \phi^{\varepsilon}-\mathbf{e}\right)+\sum_{\alpha=1}^{A} c_{0 \alpha}\left(\frac{\mathbf{x}}{\varepsilon}, \frac{\mathbf{x}}{\varepsilon^{2}}\right)\right. \\
\left.\left[f\left(s_{\alpha}\right)+f^{\prime}\left(s_{\alpha}\right)\left(\mu_{\alpha}^{\varepsilon}-F z_{\alpha} \phi^{\varepsilon}-s_{\alpha}\right)\right]\right] \mathrm{d} V
\end{array}
$$

para cada $\varepsilon$ y para cualquier campo $\mathbf{e}(\mathbf{x})$ y $s_{\alpha}(\mathbf{x})$. En particular, se considera

$$
\mathbf{e}(\mathbf{x})=\nabla \bar{\phi}(\mathbf{x})+\nabla_{y} \phi^{1}(\mathbf{x}, \mathbf{x} / \varepsilon)+\nabla_{z} \phi^{2}\left(\mathbf{x}, \mathbf{x} / \varepsilon, \mathbf{x} / \varepsilon^{2}\right) \quad \text { y } \quad s_{\alpha}(\mathbf{x})=\bar{\mu}_{\alpha}(\mathbf{x})-F z_{\alpha} \bar{\phi}(\mathbf{x})
$$

Por la definición de convergencia multi-escala, se obtiene el límite inferior

$$
\lim _{\varepsilon \rightarrow 0} I^{\varepsilon}\left[\phi^{\varepsilon}\right] \geq I\left[\bar{\phi}, \phi^{1}, \phi^{2}\right]
$$

donde

$$
\begin{aligned}
I\left[\bar{\phi}, \phi^{1}, \phi^{2}\right]= & \int_{\Omega}\left\langle\frac { 1 } { 2 } [ \nabla \overline { \phi } ( \mathbf { x } ) + \nabla _ { y } \phi ^ { 1 } ( \mathbf { x } , \mathbf { y } ) + \nabla _ { z } \phi ^ { 2 } ( \mathbf { x } , \mathbf { y } , \mathbf { z } ) ] \cdot \boldsymbol { \epsilon } ( \mathbf { y } , \mathbf { z } ) \left[\nabla \bar{\phi}(\mathbf{x})+\nabla_{y} \phi^{1}(\mathbf{x}, \mathbf{y})\right.\right. \\
& \left.\left.+\nabla_{z} \phi^{2}(\mathbf{x}, \mathbf{y}, \mathbf{z})\right]+\sum_{\alpha=1}^{A} c_{0 \alpha}(\mathbf{y}, \mathbf{z}) f\left(\bar{\mu}_{\alpha}(\mathbf{x})-F z_{\alpha} \bar{\phi}(\mathbf{x})\right)\right\rangle_{y, z} \mathrm{~d} V .
\end{aligned}
$$

Por otra parte, evaluando el funcional $I^{\varepsilon}$ en (4.18) en la secuencia de campos potenciales

$$
\begin{array}{r}
\varphi^{\varepsilon}(\mathbf{x})=\bar{\phi}(\mathbf{x})+\varepsilon \phi^{1}(\mathbf{x}, \mathbf{x} / \varepsilon)+\varepsilon^{2} \phi^{2}\left(\mathbf{x}, \mathbf{x} / \varepsilon, \mathbf{x} / \varepsilon^{2}\right)+\frac{1}{F z_{\alpha}}\left[\mu_{\alpha}^{\varepsilon}(\mathbf{x})-\bar{\mu}_{\alpha}(\mathbf{x})\right. \\
\left.-\varepsilon \mu_{\alpha}^{1}(\mathbf{x}, \mathbf{x} / \varepsilon)-\varepsilon^{2} \mu_{\alpha}^{2}\left(\mathbf{x}, \mathbf{x} / \varepsilon, \mathbf{x} / \varepsilon^{2}\right)\right]
\end{array}
$$


y apelando a la definición de convergencia de dos escalas, se obtiene el límite superior

$$
\lim _{\varepsilon \rightarrow 0} I^{\varepsilon}\left[\phi^{\varepsilon}\right] \leq \lim _{\varepsilon \rightarrow 0} I^{\varepsilon}\left[\varphi^{\varepsilon}\right]=I\left[\bar{\phi}, \phi^{1}, \phi^{2}\right]
$$

por cuanto $\phi^{\varepsilon}$ minimiza $I^{\varepsilon}$. Siendo que el límite inferior (4.21) y el límite superior (4.24) coinciden, se concluye que

$$
\lim _{\varepsilon \rightarrow 0} I^{\varepsilon}\left[\phi^{\varepsilon}\right]=I\left[\bar{\phi}, \phi^{1}, \phi^{2}\right]
$$

Consecuentemente, siguiendo los argumentos de Allaire (1992), los campos multi-escala $\bar{\phi}, \phi^{1}$ y $\phi^{2}$ deben minimizar $I\left[\bar{\phi}, \phi^{1}, \phi^{2}\right]$. Es sencillo demostrar que las condiciones de minimización implican las ecuaciones de campo (4.27) y (4.32).

\subsubsection{Problema Homogenizado}

Asumiendo que los términos de fuente $h_{\alpha}(\mathbf{x})$ son tales que las secuencias $\mu_{\alpha}^{\varepsilon}$ y $\phi^{\varepsilon}$ están acotadas en $H_{0}^{1}(\Omega)$, dichas secuencias convergen, en el sentido de convergencia multi-escala, a ciertas funciones límite $\bar{\mu}_{\alpha}(\mathbf{x})$ y $\bar{\phi}(\mathbf{x})$ en $H_{0}^{1}(\Omega)$, y sus gradientes multi-escala convergen a ciertos gradientes límite $\nabla \bar{\mu}_{\alpha}(\mathbf{x})+\nabla_{y} \mu_{\alpha}^{1}(\mathbf{x}, \mathbf{y})+\nabla_{z} \mu_{\alpha}^{2}(\mathbf{x}, \mathbf{y}, \mathbf{z})$ y $\nabla \bar{\phi}(\mathbf{x})+\nabla_{y} \phi^{1}(\mathbf{x}, \mathbf{y})+$ $\nabla_{z} \phi^{2}(\mathbf{x}, \mathbf{y}, \mathbf{z})$, donde las funciones $\mu_{\alpha}^{i} \mathrm{y} \phi^{i}$ son periódicas en $\Omega_{\#}$ en ambos argumentos (Allaire \& Briane 1996). Estas funciones son solución de las ecuaciones multi-escala

$$
\begin{aligned}
-\nabla_{z} \cdot\left[\mathrm{M}_{\alpha}(\mathbf{y}, \mathbf{z})\left(\nabla \bar{\mu}_{\alpha}(\mathbf{x})+\nabla_{y} \mu_{\alpha}^{1}(\mathbf{x}, \mathbf{y})+\nabla_{z} \mu_{\alpha}^{2}(\mathbf{x}, \mathbf{y}, \mathbf{z})\right)\right]=0 & \text { en } \Omega \times \Omega_{\#} \times \Omega_{\#}, \\
-\nabla_{z} \cdot\left[\epsilon(\mathbf{y}, \mathbf{z})\left(\nabla \bar{\phi}(\mathbf{x})+\nabla_{y} \phi^{1}(\mathbf{x}, \mathbf{y})+\nabla_{z} \phi^{2}(\mathbf{x}, \mathbf{y}, \mathbf{z})\right)\right]=0 & \text { en } \Omega \times \Omega_{\#} \times \Omega_{\#}, \\
-\nabla_{y} \cdot\left\langle\mathbb{M}_{\alpha}(\mathbf{y}, \mathbf{z})\left(\nabla \bar{\mu}_{\alpha}(\mathbf{x})+\nabla_{y} \mu_{\alpha}^{1}(\mathbf{x}, \mathbf{y})+\nabla_{z} \mu_{\alpha}^{2}(\mathbf{x}, \mathbf{y}, \mathbf{z})\right)\right\rangle_{z}=0 & \text { en } \Omega \times \Omega_{\#},
\end{aligned}
$$




$$
\begin{gathered}
-\nabla_{y} \cdot\left\langle\epsilon(\mathbf{y}, \mathbf{z})\left(\nabla \bar{\phi}(\mathbf{x})+\nabla_{y} \phi^{1}(\mathbf{x}, \mathbf{y})+\nabla_{z} \phi^{2}(\mathbf{x}, \mathbf{y}, \mathbf{z})\right)\right\rangle_{z}=0 \quad \text { en } \Omega \times \Omega_{\#}, \\
-\nabla \cdot\left\langle\mathbb{D}_{\alpha}\left(\mathbf{y}, \mathbf{z}, \bar{\mu}_{\alpha}, \bar{\phi}\right)\left(\nabla \bar{\mu}_{\alpha}(\mathbf{x})+\nabla_{y} \mu_{\alpha}^{1}(\mathbf{x}, \mathbf{y})+\nabla_{z} \mu_{\alpha}^{2}(\mathbf{x}, \mathbf{y}, \mathbf{z})\right)\right\rangle_{y, z}=h_{\alpha}(\mathbf{x}) \quad \text { en } \Omega \\
-\nabla \cdot\left\langle\epsilon(\mathbf{y}, \mathbf{z})\left(\nabla \bar{\phi}(\mathbf{x})+\nabla_{y} \phi^{1}(\mathbf{x}, \mathbf{y})+\nabla_{z} \phi^{2}(\mathbf{x}, \mathbf{y}, \mathbf{z})\right)\right\rangle_{y, z} \\
=F \sum_{\alpha=1}^{A} z_{\alpha}\left\langle c_{0 \alpha}\right\rangle_{y, z} g\left(\bar{\mu}_{\alpha}-F z_{\alpha} \bar{\phi}\right) \quad \text { en } \Omega, \\
\bar{\mu}_{\alpha}=0 \quad \text { y } \quad \bar{\phi}=0 \quad \text { en } \partial \Omega,
\end{gathered}
$$

con $\alpha=1, \ldots, A$. Y siendo

$$
\mathrm{M}_{\alpha}(\mathbf{y}, \mathbf{z})=\sum_{r=1}^{N} \chi_{1}^{(r)}(\mathbf{y}) \chi_{2}^{(r)}(\mathbf{z}) \mathbb{M}_{\alpha}^{(r)} \quad \text { con } \quad \mathbf{M}_{\alpha}^{(r)}=\frac{c_{0 \alpha}^{(r)}}{\left\langle c_{0 \alpha}\right\rangle_{y, z}} \mathbf{M}_{\alpha}^{(r)}
$$

Las ecuaciones (4.26), (4.28) y (4.30) provienen del límite multi-escala de las ecuaciones (4.8), mientras que las ecuaciones (4.27), (4.29) y (4.32) se obtienen del límite multi-escala de la ecuación (4.9). Las funciones $\bar{\mu}_{\alpha}(\mathbf{x})$ y $\bar{\phi}(\mathbf{x})$ representan los potenciales electroquímicos y eléctricos macroscópicos, mientras que las funciones $\mu_{\alpha}^{i}$ y $\phi^{i}$ representan a los potenciales microscópicos subyacentes. Nótese que las ecuaciones microscópicas/mesoscópicas (4.26)-(4.29) son lineales, y son solamente las ecuaciones macroscópicas (4.30)-(4.32) las que preservan la no linealidad de las ecuaciones de campo originales. De hecho, en el límite de homogeneización $(\varepsilon \rightarrow 0)$, los potenciales electroquímicos y eléctricos microscópicos están acoplados solamente a través de los gradientes macroscópicos. En otras palabras, para un campo eléctrico macroscópico dado, el potencial electroquímico microscópico es independiente de los potenciales electroquímicos; también, para el gradiente macroscópico de un potencial electroquímico dado, el potencial electroquímico microscópico dado es independiente de los otros potenciales electroquímicos y del potencial eléctrico. Ésta es una consecuencia de la separación de escalas y de la decomposición aditiva de las funciones (4.1) y (4.2) ya asumida. Es importante notar además que la movilidad iónica en la escala microscópica está definida por los tensores de movilidad ponderados (4.34).

Las formas de las ecuaciones (4.26)-(4.33) motivan la definición de las siguientes canti- 
dades microscópicas, mesoscópicas $(\cdot)$ y marcoscópicas $\left({ }^{-}\right)$siguientes:

$$
\begin{aligned}
& \mathbf{e}(\mathbf{x}, \mathbf{y}, \mathbf{z})=-\left[\nabla \bar{\phi}(\mathbf{x})+\nabla_{y} \phi^{1}(\mathbf{x}, \mathbf{y})+\nabla_{z} \phi^{2}(\mathbf{x}, \mathbf{y}, \mathbf{z})\right] \\
& \widetilde{\mathbf{e}}(\mathbf{x}, \mathbf{y})=\langle\mathbf{e}(\mathbf{x}, \mathbf{y}, \mathbf{z})\rangle_{z}=-\left[\nabla \bar{\phi}(\mathbf{x})+\nabla_{y} \phi^{1}(\mathbf{x}, \mathbf{y})\right] \text {, } \\
& \overline{\mathbf{e}}(\mathbf{x})=\langle\widetilde{\mathbf{e}}(\mathbf{x}, \mathbf{y})\rangle_{y}=-\nabla \bar{\phi}(\mathbf{x}), \\
& \mathbf{d}(\mathbf{x}, \mathbf{y}, \mathbf{z})=\boldsymbol{\epsilon}(\mathbf{y}, \mathbf{z}) \mathbf{e}(\mathbf{x}, \mathbf{y}, \mathbf{z}) \\
& \widetilde{\mathbf{d}}(\mathbf{x}, \mathbf{y})=\langle\mathbf{d}(\mathbf{x}, \mathbf{y}, \mathbf{z})\rangle_{z}=\widetilde{\boldsymbol{\epsilon}}(\mathbf{y}) \widetilde{\mathbf{e}}(\mathbf{x}, \mathbf{y}), \\
& \overline{\mathbf{d}}(\mathbf{x})=\langle\widetilde{\mathbf{d}}(\mathbf{x}, \mathbf{y})\rangle_{y}=\overline{\boldsymbol{\epsilon}} \overline{\mathbf{e}}(\mathbf{x}) \\
& \mathbf{j}_{\alpha}(\mathbf{x}, \mathbf{y}, \mathbf{z})=-g\left(\bar{\mu}_{\alpha}-F z_{\alpha} \bar{\phi}\right)\left\langle c_{0 \alpha}\right\rangle_{y, z} \mathbb{M}_{\alpha}(\mathbf{y}, \mathbf{z})\left[\nabla \bar{\mu}_{\alpha}(\mathbf{x})+\nabla_{y} \mu_{\alpha}^{1}(\mathbf{x}, \mathbf{y})+\nabla_{z} \mu_{\alpha}^{2}(\mathbf{x}, \mathbf{y}, \mathbf{z})\right], \\
& \widetilde{\mathbf{j}}_{\alpha}(\mathbf{x}, \mathbf{y})=\left\langle\mathbf{j}_{\alpha}(\mathbf{x}, \mathbf{y}, \mathbf{z})\right\rangle_{z}=-g\left(\bar{\mu}_{\alpha}-F z_{\alpha} \bar{\phi}\right)\left\langle c_{0 \alpha}\right\rangle_{y, z} \widetilde{\mathbf{M}}_{\alpha}(\mathbf{y})\left[\nabla \bar{\mu}_{\alpha}(\mathbf{x})+\nabla_{y} \mu_{\alpha}^{1}(\mathbf{x}, \mathbf{y})\right], \\
& \overline{\mathbf{j}}_{\alpha}(\mathbf{x})=\left\langle\widetilde{\mathbf{j}}_{\alpha}(\mathbf{x}, \mathbf{y})\right\rangle_{y}=-g\left(\bar{\mu}_{\alpha}-F z_{\alpha} \bar{\phi}\right)\left\langle c_{0 \alpha}\right\rangle_{y, z} \overline{\mathrm{M}}_{\alpha} \nabla \bar{\mu}_{\alpha}(\mathbf{x})=-\overline{\mathbb{D}}_{\alpha}\left(\bar{\mu}_{\alpha}, \bar{\phi}\right) \nabla \bar{\mu}_{\alpha}(\mathbf{x}), \\
& \bar{c}_{\alpha}(\mathbf{x})=\left\langle c_{0 \alpha}\right\rangle_{y, z} g\left(\bar{\mu}_{\alpha}-F z_{\alpha} \bar{\phi}\right) .
\end{aligned}
$$

Las segundas identidades en (4.39)-(4.43) provienen de la dependencia lineal de los campos locales $\mu_{\alpha}^{i}$ y $\phi^{i}$ en los gradientes macroscópicos $\nabla \bar{\mu}_{\alpha}$ y $\nabla \bar{\phi}$. De hecho, los problemas locales (4.26) hasta (4.29) tienen la estructura de un problema lineal estándar de conductividad. Por lo tanto, los tensores efectivos a nivel mesoscópico pueden ser expresados de la siguiente manera:

$$
\widetilde{\mathbb{M}}_{\alpha}(\mathbf{y})=\left\langle\mathbb{M}_{\alpha}(\mathbf{y}, \mathbf{z}) \mathbf{A}_{2}\left\{\mathbb{M}_{\alpha}(\mathbf{y}, \mathbf{z})\right\}\right\rangle_{z} \quad \text { y } \quad \widetilde{\boldsymbol{\epsilon}}(\mathbf{y})=\left\langle\boldsymbol{\epsilon}(\mathbf{y}, \mathbf{z}) \mathbf{A}_{2}\{\boldsymbol{\epsilon}(\mathbf{y}, \mathbf{z})\}\right\rangle_{z}
$$

mientras que los tensores efectivos a nivel macroscópico quedan dados por

$$
\overline{\mathrm{M}}_{\alpha}=\left\langle\widetilde{\mathbb{M}}_{\alpha}(\mathbf{y}) \mathbf{A}_{1}\left\{\widetilde{\mathbb{M}}_{\alpha}(\mathbf{y})\right\}\right\rangle_{y} \quad \mathrm{y} \quad \overline{\boldsymbol{\epsilon}}=\left\langle\widetilde{\boldsymbol{\epsilon}}(\mathbf{y}) \mathbf{A}_{1}\{\widetilde{\boldsymbol{\epsilon}}(\mathbf{y})\}\right\rangle_{y}
$$


En estas expresiones, los tensores $\mathbf{A}_{i}\{\cdot\}$ son los tensores estándar de concentración de los campos gradiente asociados con el problema de la celda unitaria (4.26)-(4.29) (véase, por ejemplo, Suquet 1987). Los tensores $\overline{\boldsymbol{\epsilon}}$ y $\overline{\mathrm{M}}_{\alpha}$ indican, respectivamente, la permitividad efectiva del electrolito y la movilidad efectiva de cada especie iónica $\alpha$ dentro del electrolito homogeneizado. Nótese que, para una dada escala de longitud (mesoscópica/macroscópica) todos los tensores efectivos están dados en término del mismo conjunto de tensores de concentración $\mathbf{A}_{i}$ evaluados en diferentes tensores constitutivos locales. En consecuencia, las propiedades homogenizadas a una dada escala de longitud pueden ser obtenidas resolviendo solamente un problema lineal estándar de conductividad sobre la celda unitaria. Un resultado similar ha sido obtenido recientemente en un contexto más simple por Bourbatache et al. (2013) a través de expansiones asintóticas de dos escalas. Motivados por el análisis dimensional de varias magnitudes físicas involucradas, Bourbatache et al. (2013) además consideraron un conjunto de ecuaciones microscópicas alternativas donde el miembro izquierdo de las ecuaciones de Gauss (4.27) es multiplicado por el parámetro pequeño $\varepsilon^{3}$, obteniendo así un conjunto de ecuaciones macroscópicas con la ecuación de Gauss reemplazada por la condición de electroneutralidad. Un análisis similar se puede realizar a través del método de convergencia de multi-escalas.

Con las definiciones (4.35)-(4.44), el sistema de ecuaciones multi-escala (4.26)-(4.32) puede ser escrito de la siguiente manera

$$
\begin{array}{rrr}
\nabla_{z} \cdot \mathbf{j}_{\alpha}(\mathbf{x}, \mathbf{y}, \mathbf{z})=0, & \nabla_{z} \cdot \mathbf{d}(\mathbf{x}, \mathbf{y}, \mathbf{z})=0 & \text { en } \Omega \times \Omega_{\#} \times \Omega_{\#} \\
\nabla_{y} \cdot \widetilde{\mathbf{j}}_{\alpha}(\mathbf{x}, \mathbf{y})=0, & \nabla_{y} \cdot \widetilde{\mathbf{d}}(\mathbf{x}, \mathbf{y})=0 & \text { en } \Omega \times \Omega_{\#} \\
\nabla_{x} \cdot \overline{\mathbf{j}}_{\alpha}(\mathbf{x})=h_{\alpha}(\mathbf{x}), & \nabla_{x} \cdot \overline{\mathbf{d}}(\mathbf{x})=F \sum_{\alpha=1}^{A} z_{\alpha} \bar{c}_{\alpha}(\mathbf{x}) & \text { en } \Omega .
\end{array}
$$

Finalmente, podemos definir las densidades de corriente, tanto microscópica, mesoscópica y macroscópica, como 


$$
\begin{aligned}
\mathbf{i}(\mathbf{x}, \mathbf{y}, \mathbf{z}) & =F \sum_{\alpha=1}^{A} z_{\alpha} \mathbf{j}_{\alpha}(\mathbf{x}, \mathbf{y}, \mathbf{z}), \\
\widetilde{\mathbf{i}}(\mathbf{x}, \mathbf{y}) & =\langle\mathbf{i}(\mathbf{x}, \mathbf{y}, \mathbf{z})\rangle_{z}=F \sum_{\alpha=1}^{A} z_{\alpha} \widetilde{\mathbf{j}}_{\alpha}(\mathbf{x}, \mathbf{y}), \\
\overline{\mathbf{i}}(\mathbf{x}) & =\langle\widetilde{\mathbf{i}}(\mathbf{x}, \mathbf{y})\rangle_{y}=F \sum_{\alpha=1}^{A} z_{\alpha} \overline{\mathbf{j}}_{\alpha}(\mathbf{x}) .
\end{aligned}
$$

Luego, remarcando que las concentraciones molares macroscópicas $\bar{c}_{\alpha}$ dadas por (4.44) son continuas - no así las concentraciones molares microscópicas $c_{\alpha}-$, tenemos que

$$
\overline{\mathbf{j}}_{\alpha}=-R T \sum_{\alpha=1}^{A} \overline{\mathrm{M}}_{\alpha} \nabla \bar{c}_{\alpha}+F \sum_{\alpha=1}^{A} z_{\alpha} \bar{c}_{\alpha} \overline{\mathrm{M}}_{\alpha} \overline{\mathbf{e}}
$$

y por lo tanto

$$
\overline{\mathbf{i}}=-R T F \sum_{\alpha=1}^{A} z_{\alpha} \overline{\mathrm{M}}_{\alpha} \nabla \bar{c}_{\alpha}+F^{2} \sum_{\alpha=1}^{A} z_{\alpha}^{2} \bar{c}_{\alpha} \overline{\mathrm{M}}_{\alpha} \overline{\mathbf{e}}
$$

donde hemos hecho uso de (4.43). Considerando estas expresiones, y siguiendo prácticas habituales, se pueden definir los tensores macroscópicos de transporte iónico

$$
\overline{\boldsymbol{D}}_{\alpha}=R T \overline{\mathrm{M}}_{\alpha} \quad \text { y } \quad \overline{\boldsymbol{\kappa}}=F^{2} \sum_{\alpha=1}^{A} z_{\alpha}^{2} \bar{c}_{\alpha} \overline{\mathrm{M}}_{\alpha}
$$

que representan respectivamente la difusividad efectiva y la conductividad efectiva del electrolito microestructurado. Vale la pena remarcar que la conductividad depende de las concentraciones iónicas $\bar{c}_{\alpha}$ y por ende no es un parámetro material. 


\section{Capítulo 5}

\section{Problema Modelo Lineal}

\subsection{Problema modelo de transporte iónico lineal}

En esta sección nos hemos enfocado particularmente en electrolitos poliméricos semicristalinos que poseen microestructuras esferulíticas y operan a temperatura ambiente. Para tener cierta especificidad en los modelos desarrollados se ha considerando como caso de estudio un sistema de PEO dopado con $\mathrm{LiClO}_{4}$ como el descripto, por ejemplo, por FullertonShirey \& Maranas (2009). En estos sistemas las moléculas de PEO disuelven las sales de $\mathrm{LiClO}_{4}$ en $\mathrm{Li}^{+}(\alpha=+)$ y $\mathrm{ClO}_{4}^{-}(\alpha=-)$, ambos iones univalentes $\left(z_{+}=-z_{-}=1\right)$ y forman varias fases dependiendo de la concentración de sales y la temperatura. A temperatura ambiente, la morfología microestructural presenta una estructura de dos escalas. En su escala menor, una escala nanométrica, se conforma un laminado integrado por capas alternadas de fase amorfa y fase cristalina; dichos laminados se disponen de manera radial desde puntos nucleantes en el especimen, resultando en estructuras llamadas esferulitas, que conforman la escala mayor en el orden de los micrómetros. Dependiendo de la concentración de sales, las esferulitas pueden formar agregados granulares o estar separados por una matriz continua de fase amorfa y por ende la fracción volumétrica de la fase cristalina puede variar entre un $10 \%$ y un $90 \%$. En la escala molecular, la fase cristalina consiste en celdas unitarias monoclínicas formadas por cadenas poliméricas que exiben una configuración helicoidal (Gitelman et al. 


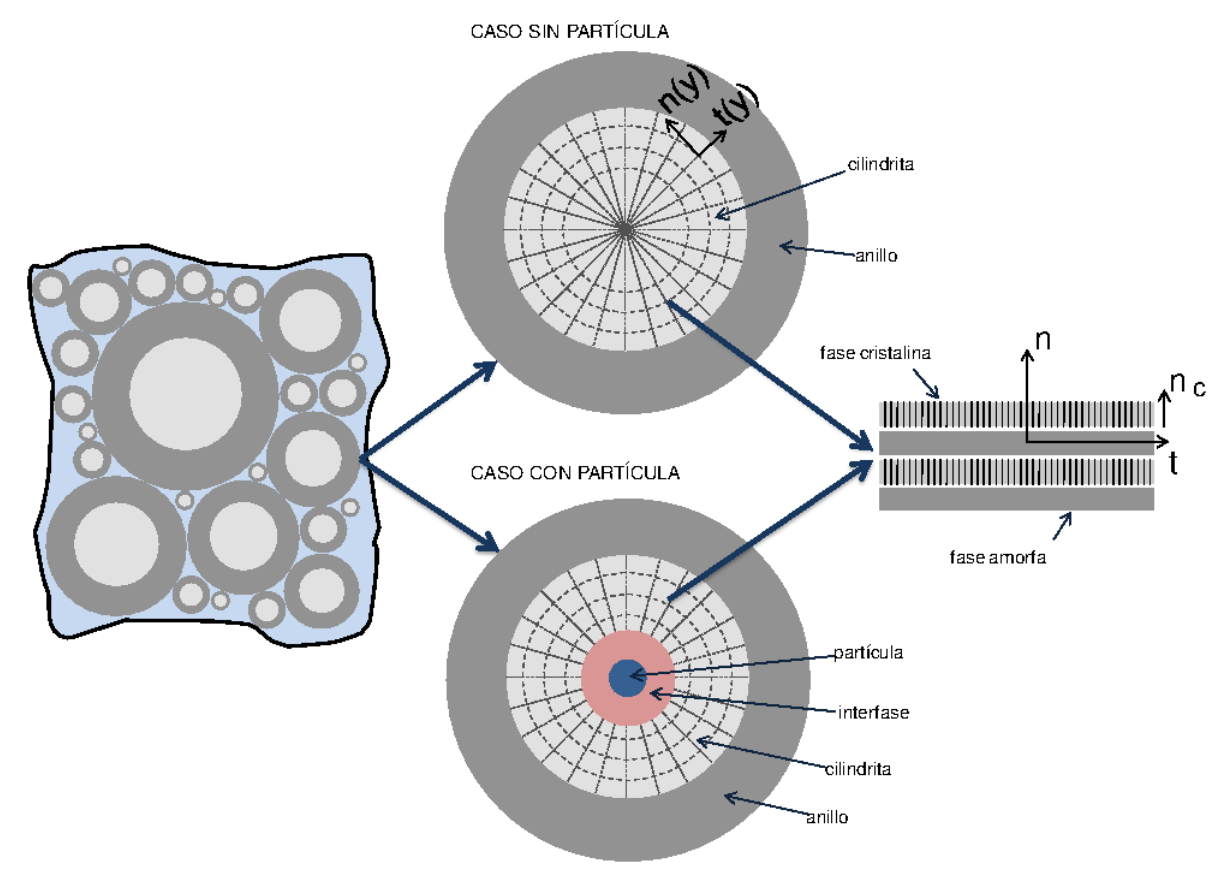

Figura 5.1: Esquema del ensamble de cilindros de Schulgasser utilizado en los modelos con y sin inclusiones. 2008).

Hemos derivado un modelo multi-escala para estos sistemas materiales construyendo microgeometrías bi-dimensionales que, por un lado son suficientemente complejas para reproducir las características esenciales geométricas, pero por otro lado son suficientemente simples de modo que la respuesta homogenizada pueda ser calculada de manera exacta. La microgeometría seleccionada consiste de una combinación de un ensamble de cilindros de Schulgasser (Schulgasser 1983) y laminados simples. En la meso-escala, estas microgeometrías son ensambles de cilindros homotéticos con infinitas escalas, que completan todo el espacio de la celda unitaria, cada uno compuesto de una capa exterior isótropa de fase amorfa y un núcleo interior radialmente simétrico llamado cilindrita. En la micro-escala, la cilindrita es a su vez una microgeometría laminada compuesta de laminados alternados de fases amorfas y cristalinas con la dirección de laminación coincidente con la dirección tangencial de la meso-escala. Un esquema de la microgeometría es presentado en la Figura 5.1 .

Los parámetros microestructurales son la fracción volumétrica de los cilindros que es ocupada por el anillo exterior amorfo $f_{r}$ y por las cilindritas $f_{c}$, y las fracciones volumétricas de la estructura lamelar dentro de la cilindrita que es ocupada por la fase amorfa $f_{c}^{a}$ y por 
la fase cristalina $f_{c}^{c r}$. Estos parámetros satisfacen las siguientes identidades

$$
f_{r}+f_{c}=1, \quad \mathrm{y} \quad f_{c}^{a}+f_{c}^{c r}=1
$$

La fracción volumétrica total de las fases cristalinas y amorfas son dadas por

$$
f^{(c r)}=f_{c} f_{c}^{c r} \quad \text { y } \quad f^{(a)}=f_{c} f_{c}^{a}+f_{r}
$$

respectivamente, y son tal que $f^{(c r)}+f^{(a)}=1$; la fracción volumétrica $f^{(c r)} \equiv f$ representa la cristalinidad del electrolito.

Los tensores de movilidad de cada clase de iones en la fase amorfa isótropa y en la fase cristalina anisótropa quedan, respectivamente, expresados como

$$
\mathbf{M}_{\alpha}^{(a)}=m_{\alpha}^{(a)} \mathbf{I} \quad \mathrm{y} \quad \mathbf{M}_{\alpha}^{(c r)}=m_{\alpha}^{\|} \mathbf{n}_{c} \otimes \mathbf{n}_{c}+m_{\alpha}^{\perp}\left(\mathbf{I}-\mathbf{n}_{c} \otimes \mathbf{n}_{c}\right)
$$

donde $m_{\alpha}^{\|}$y $m_{\alpha}^{\perp}$ representan las movilidades a lo largo de la dirección paralela y perpendiculares a la dirección de los ejes de las cadenas helicoidales $\mathbf{n}_{c}$. Los tensores de movilidad ponderados $\mathbb{M}_{\alpha}$ están dados por

$$
\mathbb{M}_{\alpha}^{(a)}=\not h_{\alpha}^{(a)} \mathbf{I} \quad \mathrm{y} \quad \mathbb{M}_{\alpha}^{(c r)}=\not h_{\alpha}^{\|} \mathbf{n}_{c} \otimes \mathbf{n}_{c}+\not h_{\alpha}^{\perp}\left(\mathbf{I}-\mathbf{n}_{c} \otimes \mathbf{n}_{c}\right)
$$

con

$$
\not h_{\alpha}^{(a)}=\frac{c_{0 \alpha}^{(a)}}{f^{(c r)} c_{0 \alpha}^{(c r)}+f^{(a)} c_{0 \alpha}^{(a)}} m_{\alpha}^{(a)} \quad \text { y } \quad \not h_{\alpha}^{\|, \perp}=\frac{c_{0 \alpha}^{(c r)}}{f^{(c r)} c_{0 \alpha}^{(c r)}+f^{(a)} c_{0 \alpha}^{(a)}} m_{\alpha}^{\|, \perp}
$$

Aquí, $c_{0 \alpha}^{(c r)}$ and $c_{0 \alpha}^{(a)}$ son las concentraciones de referencia de las fases cristalina y amorfa, respectivamente.

Siguiendo el esquema de la sección anterior, la respuesta efectiva es obtenida secuencialmente, primero homogenizando la estructura laminar para obtener las movilidades mesoscópicas $\widetilde{\mathbb{M}}_{\alpha}$ como es indicado en $(4.45)_{1}$, y luego homogenizando el ensamble de Schulgasser para obtener las movilidades macroscópicas $\overline{\mathrm{M}}_{\alpha}$ como es detallado en (4.46) ${ }_{1}$. Al hacerlo, 
es importante destacar que, estrictamente, las geometrías multi-escala consideradas aquí no están incluídas en la clase de microgeometrías consideradas en la sección previa, donde es requerido que la celda unitaria en ambas escalas sean cúbicas y mutuamente alineadas. Sin embargo, es razoanable asumir que las ecuaciones multi-escala (4.35)-(4.44) mantienen su validez más generalmente.

El análisis subsiguiente se simplifica considerablemente asumiendo que los ejes de las cadenas $\mathbf{n}_{c}$ están alineados con la dirección de laminación $\mathbf{n}$. Esto equivale a ignorar la inclinación de las cadenas. Asumiendo ésto, las movilidades efectivas en la estructura lamelar pueden ser escritas de la siguiente manera — véase, por ejemplo, Milton (2002)—

$$
\widetilde{\mathbb{M}}_{\alpha}(\mathbf{y})=\widetilde{\not h}_{\alpha}^{\|} \mathbf{n}(\mathbf{y}) \otimes \mathbf{n}(\mathbf{y})+\widetilde{\not h}_{\alpha}^{\perp}[\mathbf{I}-\mathbf{n}(\mathbf{y}) \otimes \mathbf{n}(\mathbf{y})]
$$

donde $\mathbf{n}(\mathbf{y})$ es la dirección de laminación en la posición y dentro del ensamble de Schulgasser, y las movilidades paralela y perpendicular están dadas por

$$
\widetilde{\not h}_{\alpha}^{\|}=\left(\frac{f_{c}^{c r}}{\not h_{\alpha}^{\|}}+\frac{f_{c}^{a}}{\not h_{\alpha}^{(a)}}\right)^{-1} \quad \text { y } \quad \widetilde{\not h}_{\alpha}^{\perp}=f_{c}^{c r} \not h_{\alpha}^{\perp}+f_{c}^{a} \not h_{\alpha}^{(a)} .
$$

Consecuentemente, los ejes anisótropos de los tensores de movilidad mesoscópicos están radialmente alineados dentro de las cilindritas. Esto permite el uso de las soluciones de Schulgasser para obtener los tensores de movilidad macrocópicos $\overline{\mathrm{M}}_{\alpha}$, que son

$$
\overline{\mathrm{M}}_{\alpha}=\overline{\not h}_{\alpha} \mathbf{I}
$$

Para poder arribar a dichas soluciones se debe considerar un ensamble de Shulgasser compuesto de cilindros homotéticos con movilidades radiales y tangenciales $m^{\|}(\rho)$ y $m^{\perp}(\rho)$, arbitrariamente variables con la coordenada radial $\rho \in[0,1]$ dentro de los cilindros. Omitiremos para estas consideraciones los subíndices $\alpha$ que indican la especie iónica. Los valores extremos $\rho=0$ y $\rho=1$ corresponden respectivamente al centro y al límite exterior del cilindro. Schulgasser (1983) demostró ${ }^{1}$ que la movilidad efectiva $\bar{m}$ del ensamble está determinado

\footnotetext{
${ }^{1}$ Schulgasser (1983) consideró en su trabajo original el problema de conductividad térmica, matemáticamente análogo al aquí analizado.
} 
por — véase también Milton (2002)—

$$
\bar{m}=h(1)
$$

donde la función $h(\rho) \in C^{0}[0,1]$ es solución de la ecuación de Ricatti

$$
\rho h^{\prime}(\rho)+\frac{[h(\rho)]^{2}}{m^{\perp}(\rho)}=m^{\|}(\rho)
$$

Considérese ahora el caso de un cilindro con un núcleo anisótropo de radio $\rho_{c}$ con movilidades $m_{c}^{\|, \perp}$ y un anillo externo isótropo de movilidad $m_{r}$. Luego, se puede plantear que

$$
m^{\|, \perp}(\rho)= \begin{cases}m_{c}^{\|, \perp} & 0 \leq \rho \leq \rho_{c} \\ m_{r} & \rho_{c}<\rho \leq 1\end{cases}
$$

Integrando (5.10) por partes y haciendo cumplir la continuidad en $\rho=\rho_{c}$ se obtiene

$$
h(\rho)= \begin{cases}\sqrt{m_{c}^{\|} m_{c}^{\perp}} & 0 \leq \rho \leq \rho_{c}, \\ m_{r} \frac{\left(\sqrt{m_{c}^{\|} m_{c}^{\perp}}+m_{r}\right)\left(\rho / \rho_{c}\right)^{2}+\left(\sqrt{m_{c}^{\|} m_{c}^{\perp}}-m_{r}\right)}{\left(\sqrt{m_{c}^{\|} m_{c}^{\perp}}+m_{r}\right)\left(\rho / \rho_{c}\right)^{2}-\left(\sqrt{m_{c}^{\|} m_{c}^{\perp}}-m_{r}\right)} & \rho_{c}<\rho \leq 1 .\end{cases}
$$

En $\rho=1$ se tiene que $\left(\rho / \rho_{c}\right)^{2}=1 / f_{c}$ donde $f_{c}$ es la fracción volumétrica del núcleo. De esta manera, luego de operar se obtiene

$$
\bar{m}=h(1)=m_{r} \frac{\left(1+f_{c}\right) \sqrt{m_{c}^{\|} m_{c}^{\perp}}+\left(1-f_{c}\right) m_{r}}{\left(1-f_{c}\right) \sqrt{m_{c}^{\|} m_{c}^{\perp}}+\left(1+f_{c}\right) m_{r}} .
$$

Siguiendo las ecuaciones (5.7), considerando las movilidades efectivas del laminado y utilizando la expresión (5.13) en la cual retomamos la presencia de los subíndices $\alpha$ para cada 
especie iónica, se tiene que

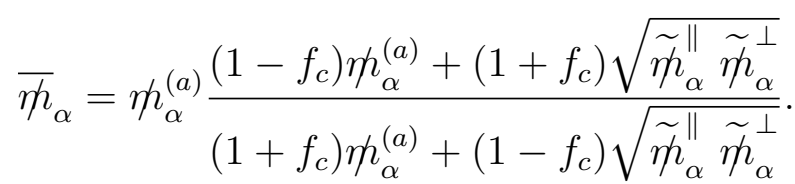

La expresion (5.14), junto con (5.5) y (5.7), constituyen una estimación autocontenida para las movilidades de cada especie iónica a través del modelo del polímero semi-cristalino en términos de las movilidades locales de las fases amorfas y cristalinas, y de varios parámetros microestructurales. En ausencia de anillos amorfos $\left(f_{c}=1\right)$, dicha expresión se reduce a

$$
\overline{\not h}_{\alpha}=\sqrt{\widetilde{\not h}_{\alpha}^{\|} \widetilde{\not h}_{\alpha}^{\perp}}
$$

Si, además no hay fase amorfa presente en la estructura laminal $\left(f_{c}=f_{c}^{c r}=f^{(c r)}=1\right)$, la expresión se reduce aún más a

$$
\overline{\eta ̆}_{\alpha}=\sqrt{m_{\alpha}^{\|} m_{\alpha}^{\perp}} \text {. }
$$

Nótese que en todos los casos, $\overline{\not h}_{\alpha}$ es simétrico en $m_{\alpha}^{\|}$y $m_{\alpha}^{\perp}$ : intercambiar las movilidades paralela y perpendicular en la fase cristalina deviene en obtener la misma movilidad efectiva.

Dada la isotropía general del electrolito, la conductividad efectiva es de la forma $\overline{\boldsymbol{\kappa}}=\bar{\kappa} \mathbf{I}$. En una batería de litio convencional son sólo los cationes los que reaccionan químicamente en la interfaz electrodo - electrolito. De esta manera, bajo condiciones estacionarias no hay flujo neto de aniones a través del electrolito y la conducción iónica se debe únicamente al transporte de cationes. Consecuentemente definimos

$$
\bar{\kappa}=F^{2} \bar{c}_{+} \overline{\eta h}_{+} .
$$

Es conveniente, para el desarrollo posterior introducir una concentración molar de referencia $c_{0}=\rho_{P E O} / w_{P E O}$, donde $\rho_{P E O}$ y $w_{P E O}$ representan la densidad de masa y el peso molar del $\mathrm{PEO}$, respectivamente, y una conductividad de referencia $\kappa_{0}=F^{2} z_{+}^{2} c_{0} m_{+}^{(a)}$, de tal manera 
que

$$
\frac{\bar{\kappa}}{\kappa_{0}}=\frac{\bar{c}_{+}}{c_{0}} \frac{\overline{\not h}}{m_{+}^{(a)}} .
$$

Finalmente, se observa que si los tensores de permitividad eléctrica de las fases amorfas y cristalinas son de la misma forma (5.3), el tensor de permitividad efectiva $\overline{\boldsymbol{\epsilon}}$ del electrolito está dado por las mismas expresiones (5.8)-(5.14) con las movilidades pesadas reemplazadas por las permitividades eléctricas locales.

\subsection{El efecto de la cristalinidad y la concentración de sales}

En esta sección se presenta un conjunto de resultados numéricos con el objetivo de estudiar la dependencia que los numerosos parámetros materiales y microestructurales tienen sobre las movilidades macroscópicas. Comenzamos por considerar sistemas materiales sin anillo amorfo $\left(f_{r}=0\right)$. Los resultados están parametrizados con el contraste de concentraciones de referencia $c_{0 \alpha}^{(c r)} / c_{0 \alpha}^{(a)}$, el contraste en las movilidades $\left(m_{\alpha}^{\|}+m_{\alpha}^{\perp}\right) /\left(2 m_{\alpha}^{(a)}\right)$, y la relación de anisotropía de las movilidades de la fase cristalina $m_{\alpha}^{\|} / m_{\alpha}^{\perp}$. Se asumen dos tipos de relaciones entre las movilidades de cada fase, en el primer grupo de resultados, presentado en la Figura 5.2 las movilidades son tales que $m_{\alpha}^{\perp} \leq m_{\alpha}^{a} \leq m_{\alpha}^{\|}$, en el segundo grupo, el de la Figura 5.3 se utilizó $m_{\alpha}^{\|} \leq m_{\alpha}^{a} \leq m_{\alpha}^{\perp}$. Por otra parte, dado que la relación entre los éteres de oxígeno y los iones de litio de la fase cristalina son típicamente menores que en la fase amorfa, se asume que $c_{0 \alpha}^{(c r)} / c_{0 \alpha}^{(a)} \geq 1$. Las Figuras 5.2 y 5.3 muestran predicciones para la movilidad efectiva $\bar{m}_{\alpha}$ de las especies $\alpha$, normalizada con la correspondiente movilidad de la fase amorfa $m_{\alpha}^{(a)}$, como función de la cristalinidad $f$, para un amplio rango de parámetros materiales. Dichos resultados adimensionalizados son los mismos para ambas especies de iones univalentes $\alpha$ para el caso en estudio. La principal observación en el contexto de estas Figuras es que la movilidad efectiva puede decrecer con un aumento de la cristalinidad aún cuando la movilidad de la fase cristalina, ya sea en el sentido de los ejes de las cadenas o en su sentido perpendicular, es incluso varios órdenes de magnitud superior que la movilidad de la fase amorfa. De hecho, las predicciones presentadas en las Figuras (a) y (b) de ambos 
a)

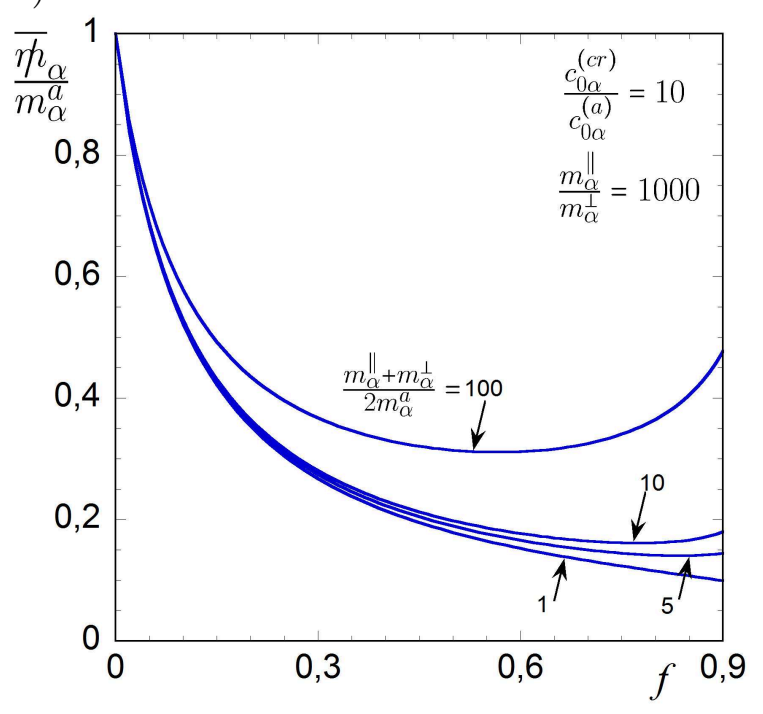

c)

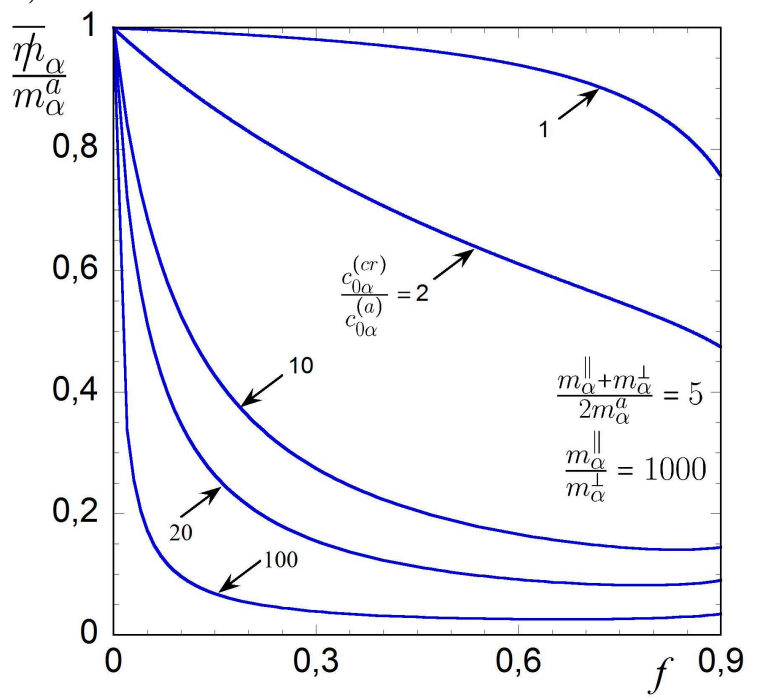

b)

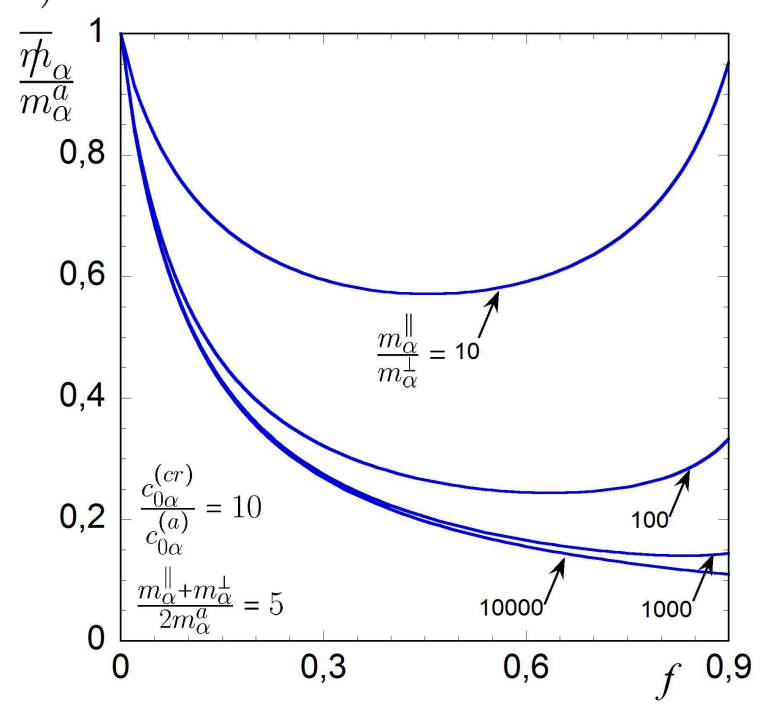

d)

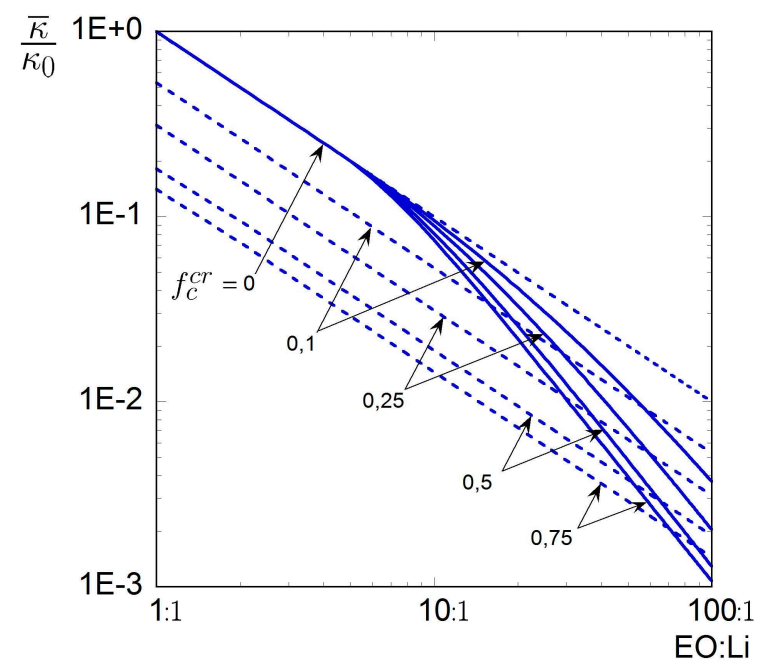

Figura 5.2: (a)-(c) Movilidad efectiva de las especies $\alpha$ en un electrolito polimérico semi-cristalino sin anillos amorfos $\left(f_{r}=0\right)$, normalizada con la correspondiente movilidad $m_{\alpha}^{(a)}$ de la fase amorfa, versus cristalinidad $f$, para el caso de $m_{\alpha}^{\|}>m_{\alpha}^{\perp}$; gráficas para: (a) $c_{0 \alpha}^{(c r)} / c_{0 \alpha}^{(a)}=10, m_{\alpha}^{\|} / m_{\alpha}^{\perp}=1000$, y diferentes contrastes en las movilidades $\left(m_{\alpha}^{\|}+m_{\alpha}^{\perp}\right) /\left(2 m_{\alpha}^{(a)}\right)$; (b) $c_{0 \alpha}^{(c r)} / c_{0 \alpha}^{(a)}=10,\left(m_{\alpha}^{\|}+m_{\alpha}^{\perp}\right) /\left(2 m_{\alpha}^{(a)}\right)=5$, y diferentes proporciones de anisotropía en la movilidad $m_{\alpha}^{\|} / m_{\alpha}^{\perp}$; (c) $\left(m_{\alpha}^{\|}+m_{\alpha}^{\perp}\right) /\left(2 m_{\alpha}^{(a)}\right)=5, m_{\alpha}^{\|} / m_{\alpha}^{\perp}=1000$, y varios contrastes en las concentraciones de referencia $c_{0 \alpha}^{(c r)} / c_{0 \alpha}^{(a)}$. (d) Conductividad efectiva de una clase de electrolitos con microestructura variable, normalizada con una conductividad de referencia $\kappa_{0}$, en función de la relación de concentración de sales de litio por cada mol de PEO, para diferentes cristalinidades de las cilindritas $f_{c}^{c r}$.

casos muestran que para contrastes en la movilidad suficientemente bajos $(\leq 5)$ y grandes relaciones de anisotrpía en las movilidades $(\geq 1000)$ la movilidad efectiva desciende, hasta un orden de magnitud, de manera monótona con la cristalinidad en el rango de $0 \leq f \leq 0,9$. 
a)

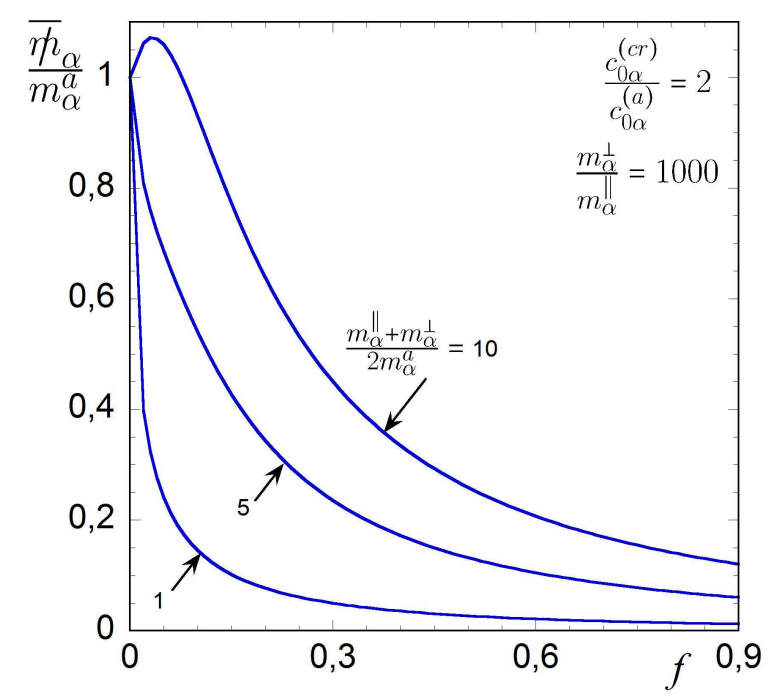

c)

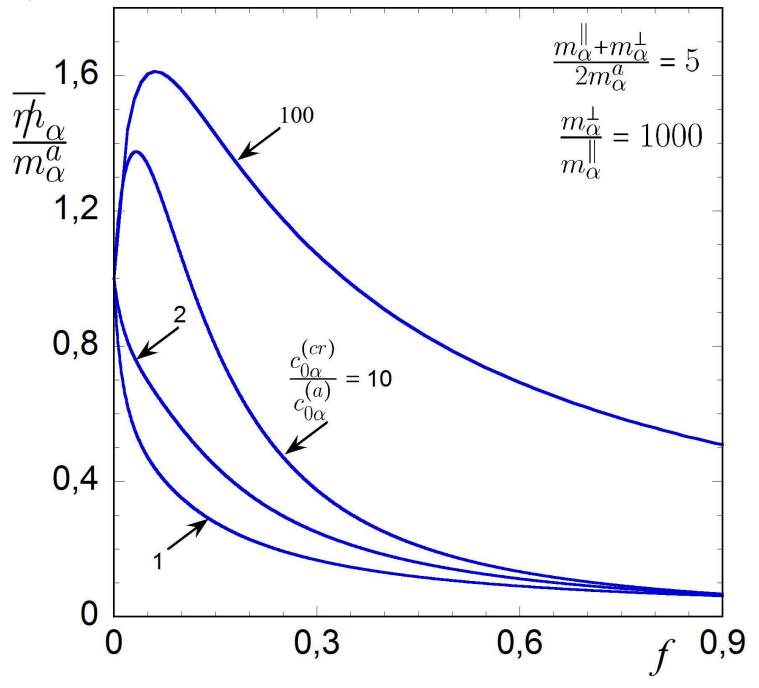

b)

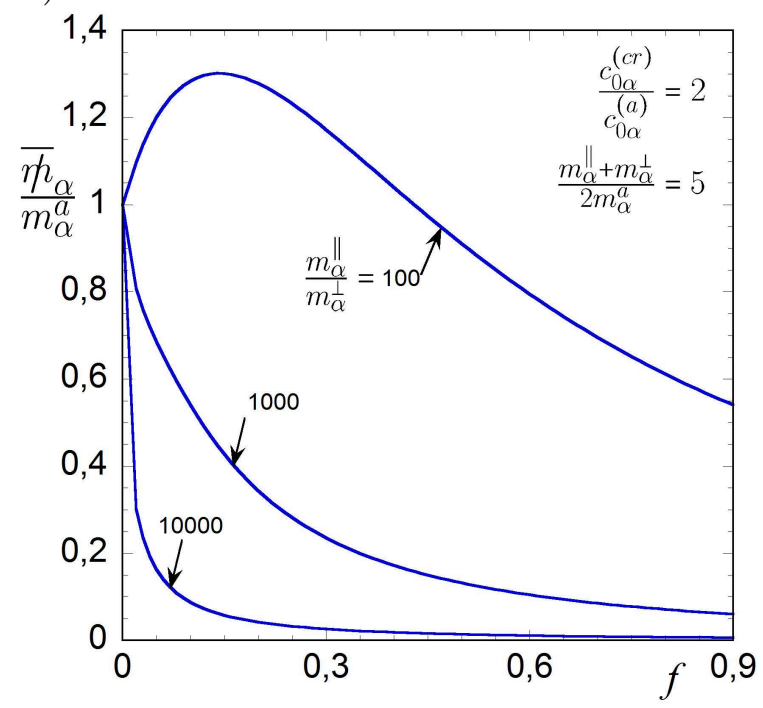

d)

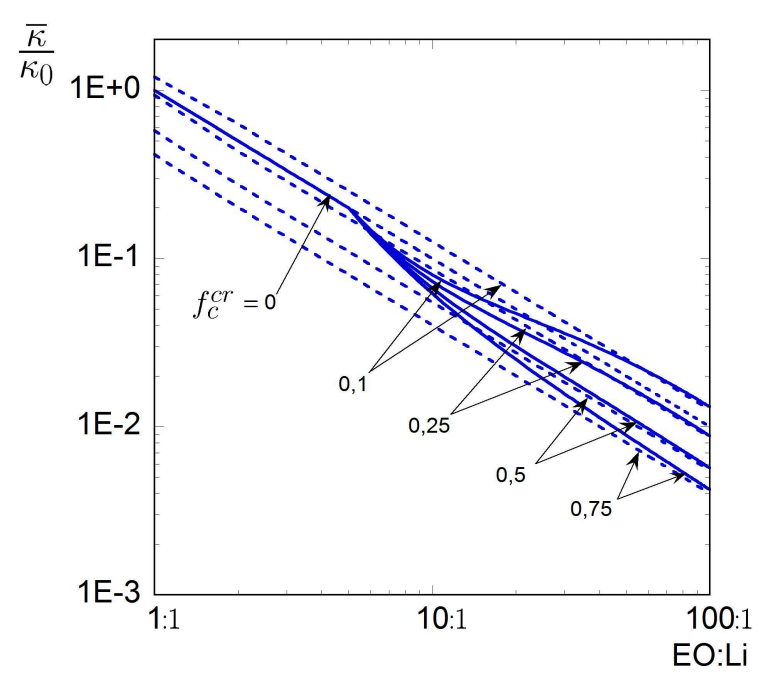

Figura 5.3: (a)-(c) Movilidad efectiva de las especies $\alpha$ en un electrolito polimérico semi-cristalino sin anillos amorfos $\left(f_{r}=0\right)$, normalizada con la correspondiente movilidad $m_{\alpha}^{(a)}$ de la fase amorfa, versus cristalinidad $f$, para el caso de $m_{\alpha}^{\perp}>m_{\alpha}^{\|}$; gráficas para: (a) $c_{0 \alpha}^{(c r)} / c_{0 \alpha}^{(a)}=2, m_{\alpha}^{\|} / m_{\alpha}^{\perp}=1000$, y diferentes contrastes en las movilidades $\left(m_{\alpha}^{\|}+m_{\alpha}^{\perp}\right) /\left(2 m_{\alpha}^{(a)}\right)$; (b) $c_{0 \alpha}^{(c r)} / c_{0 \alpha}^{(a)}=2,\left(m_{\alpha}^{\|}+m_{\alpha}^{\perp}\right) /\left(2 m_{\alpha}^{(a)}\right)=5$, y diferentes proporciones de anisotropía en la movilidad $m_{\alpha}^{\|} / m_{\alpha}^{\perp}$; (c) $\left(m_{\alpha}^{\|}+m_{\alpha}^{\perp}\right) /\left(2 m_{\alpha}^{(a)}\right)=5, m_{\alpha}^{\|} / m_{\alpha}^{\perp}=1000$, y varios contrastes en las concentraciones de referencia $c_{0 \alpha}^{(c r)} / c_{0 \alpha}^{(a)}$. (d) Conductividad efectiva de una clase de electrolitos con microestructura variable, normalizada con una conductividad de referencia $\kappa_{0}$, en función de la relación de concentración de sales de litio por cada mol de PEO, para diferentes cristalinidades de las cilindritas $f_{c}^{c r}$.

Esto muestra que la reducción de la conductividad iónica con el aumento de la cristalinidad, comunmente observada en polímeros semi-cristalinos no implica un mejor transporte de iones en la fase amorfa respecto al de la fase cristalina, como fue originalmente propuesto 
por Berthier et al. (1983). Por otra parte, si bien este modelo no contempla el efecto de la deformación mecánica, es evidente que las predicciones presentadas proveen una posible explicación mecánica para el aumento de la conductividad iónica observado en electrolitos deformados (Golodnitsky \& Peled 2000; Golodnitsky et al., 2001; Minami et al., 2005). De hecho, deformando por tracción un material de las características antes mencionadas, las cadenas poliméricas tanto de la fase amorfa como de la cristalina tienden a alinearse con la dirección de la deformación (Burba et al., 2011); la movilidad efectiva a lo largo de la dirección de estiramiento debe por lo tanto tender a $m_{\alpha}^{\|}$. Para el caso de $c_{0 \alpha}^{(c r)} / c_{0 \alpha}^{(a)}=10$, $m_{\alpha}^{\|} / m_{\alpha}^{\perp}=1000 \mathrm{y}\left(m_{\alpha}^{\|}+m_{\alpha}^{\perp}\right) /\left(2 m_{\alpha}^{(a)}\right)=5$, la movilidad $m_{\alpha}^{\|}$puede ser dos órdenes de magnitud mayor que la movilidad efectiva predicha para sistemas sin deformar con niveles de cristalinidad moderados. Este es aproximadamente el incremento reportado por Gitelman et al. (2008) en sistemas de PEO dopados con LiI.

Las predicciones provistas en la Figura 5.2-c muestran el rol del contraste en las concentraciones de referencia en la determinacion de la movilidad efectiva, para el caso de $m_{\alpha}^{\|} / m_{\alpha}^{\perp}=1000$ y $\left(m_{\alpha}^{\|}+m_{\alpha}^{\perp}\right) /\left(2 m_{\alpha}^{(a)}\right)=5$. Puede observarse que las tendencias más aproximadas a la realidad se dan para $c_{0 \alpha}^{(c r)}>2 c_{0 \alpha}^{(a)}$. Por ende, la hipótesis habitual de $c_{0 \alpha}^{(c r)}=c_{0 \alpha}^{(a)}$, que implica que las concentraciones de iones son continuas y consecuentemente simplifican la formulación matemática, está en desacuerdo no sólo con la heterogeneidad de la concentración local de iones en equilibrio esperada sino además con la variación macroscópica de la conductividad con la cristalinidad observada experimentalmente.

En este punto, debe ser resaltado que electrolitos poliméricos con diferentes conductividades efectivas siempre difieren en varias características materiales y microestructurales, resultando dificultoso aislar experimentalmente el efecto individual de cada uno de los múltiples parámetros. Por ejemplo, el trabajo de Fullerton-Shirey \& Maranas (2009) sugiere que, a medida que la concentración de sales en el casteo aumenta, la fase amorfa que rodea a las esferulitas ocupa mayor fracción volumétrica del electrolito, y que la concentración de sales en la fase cristalina se mantiene constante y el aumento de la misma sólo se da en la fase amorfa. A manera de ejemplo, en la Figura 5.2-d se muestran predicciones para los valores de la conductividad efectiva en función de la concentración molar de sales de litio en el casteo $c$, considerando dichas variaciones en la microestructura y los parámetros materiales. Notar 
que la relación $\left(c / c_{0}\right)^{-1}$ representa la relación total de los éteres de oxígeno del PEO respecto a los iones de litio (EO:Li) en el electrolito recién casteado. Las predicciones corresponden a $\bar{c}_{+}=c$ y a movilidades del litio tal que $m_{+}^{\|} / m_{+}^{\perp}=1000 \mathrm{y}\left(m_{+}^{\|}+m_{+}^{\perp}\right) /\left(2 m_{+}^{a}\right)=5$. El volumen de la fracción de anillo amorfo y la concentración de referencia han sido tomados como funciones de $c$ como

$$
f_{r}=\gamma_{r}\left(\frac{c}{c_{0}}\right) \quad \text { y } \quad \frac{c_{0+}^{(c r)}}{c_{0+}^{(a)}}=\gamma_{c}\left(\frac{c}{c_{0}}\right)^{-1}
$$

con $\gamma_{r}=1 / \gamma_{c}=5$; estas constantes numéricas provienen de datos aproximados del trabajo de Fullerton-Shirey \& Maranas (2009). A modo de referencia, la Figura también incluye resultado para sistemas sin anillo amorfo $\left(f_{r}=0\right)$ y con $c_{0+}^{(c r)} / c_{0+}^{(a)}=10$ fijo (líneas de trazos). La principal obsrvación en el contexto de este gráfico es que la variación de los parámetros materiales y microestructurales del tipo (5.19) pueden aumentar la variabilidad de la conductividad efectiva con la concetración de sales en un orden de magnitud. Véase que para $f_{c}^{c r}=0,75$ la variación es de tres órdenes de magnitud, lo cual cae dentro del rango observado por Fullerton-Shirey \& Maranas (2009). El quiebre en estas curvas corresponde a relaciones de EO:Li por debajo de las cuales el material es completamente amorfo. Debemos resaltar, sin embargo, que pueden ocurrir cambios microestructurales adicionales, lo cual seguramente modificaría las tendencias presentadas. Por ejemplo, las moleculas de la sal de litio agregadas al polímero podrían segregarse, para los casos de altas concentraciones, y formar partículas de una segunda fase (ej., Marzantowicz et al. 2008). El marco teórico ya presentado puede por cierto incorporar dichas modificaciones, siempre y cuando suficientes observaciones experimentales pertinentes al caso estén disponibles y permitan realizar hipótesis sobre dichos parámetros.

Para los casos donde $m_{\alpha}^{\|} \leq m_{\alpha}^{a} \leq m_{\alpha}^{\perp}$, presentados en la Figura 5.3, son también válidas las observaciones realizadas previamente. Sobre todo es importante recalcar que en estos casos también es factible obtener una movilidad efectiva decreciente ante un aumento de la cristalinidad, incluso siendo que la fase cristalina presente una de sus dos componentes de movilidad anisótropa mayor a la de la fase amorfa. Es preciso también agregar que para ciertos valores de anisotropías, tanto en la movilidad como en las concentraciones de refe- 
rencia, en el caso en que la movilidad de la fase amorfa en el sentido perpendicular a las cadenas sea mayor a la del sentido longitudinal, es posible obtener valores de movilidad efectiva superiores a la movilidad de la fase amorfa. Esto podría ser consistente con algunas de las observaciones experimentales ya citadas donde reduciendo la critalinidad se obtienen movilidades efectivas superiores. Estos resultados, como ya dijimos y como se desprende de las figuras, no implican que una mayor cristalinidad necesariamente devenga en una menor movilidad efectiva, pero podrían ser uno de los motivos de los numerosos trabajos que buscan reducir las cristalinidades en este tipo de electrolitos sólidos.

\subsection{El efecto de inclusiones aislantes}

Además de la conductividad iónica, el número de transporte

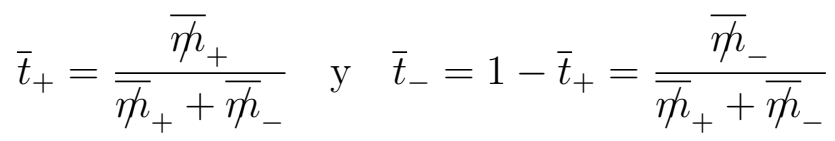

es una propiedad de particular relevancia para el desempeño de una batería — véase, por ejemplo, Doyle et al. (1994). En general, es deseable que el número de tranporte para el catión $\left(\bar{t}_{+}\right)$sea alto. En sistemas de PEO dopados con $\mathrm{LiClO}_{4}$, el número de transporte del catión suele ser relativamente bajo ( $\sim 0,25$ a temperatura ambiente) pero el mismo se incrementa significativamente con la adición de partículas cerámicas como $\mathrm{Al}_{2} \mathrm{O}_{3}$ y $\mathrm{ZrO}_{2}$ —véase, como referencia, Croce et al. (2001,2006), Ciocek et al. (2007). El modelo de ensambles de Schulgasser planteado anteriormente puede ser modificado de manera de tener la capacidad de evaluar el rol de las interfases en este efecto.

En este caso, se asume que las cilindritas contienen una inclusión central donde las movilidades y concentraciones de referencia son nulas, representando de esta manera la partícula no conductiva, rodeada de una capa isótropa con movilidades $m_{\alpha}^{(i n t)}$ y concentraciones de referencia $c_{0 \alpha}^{(i n t)}$, véase la Figura 5.1. Dicha microgeometría fue elegida de manera consistente con las observaciones que indican que las partículas agragadas a estos sistemas actúan de puntos de nucleación para las esferulitas en el casteo del polímero y que además están rodeadas por interfases amorfas de dimensiones finitas (ej., Siekierski et al. 2007). Para obtener la 
movilidad efectiva de dichos ensambles se procede de manera similar al apartado anterior. Para ello considérese ahora el caso de un cilindro conformado por una inclusión central rodeada por una interfase concéntrica isótropa. Omitiremos nuevamente los subíndices $\alpha$ que indican la especie iónica. Luego,

$$
m^{\|, \perp}(\rho)= \begin{cases}m_{\text {inc }} & 0 \leq \rho<\rho_{\text {inc }} \\ m_{\text {int }} & \rho_{\text {inc }} \leq \rho<\rho_{\text {int }} \\ m_{c}^{\|, \perp} & \rho_{\text {int }} \leq \rho<\rho_{c} \\ m_{r} & \rho_{c} \leq \rho \leq 1 .\end{cases}
$$

Integrando (5.10) por partes y asegurándose que se cumpla la continuidad de la solución se obtiene

$$
h(\rho)= \begin{cases}m_{i n c} & 0 \leq \rho \leq \rho_{i n c}, \\ m_{i n t} \frac{\left(m_{i n c}+m_{i n t}\right)\left(\rho / \rho_{i n c}\right)^{2}+\left(m_{i n c}-m_{i n t}\right)}{\left(m_{i n c}+m_{i n t}\right)\left(\rho / \rho_{i n c}\right)^{2}-\left(m_{i n c}-m_{i n t}\right)} & \rho_{i n c}<\rho \leq \rho_{i n t}, \\ \sqrt{m_{c}^{\|} m_{c}^{\perp} \frac{\left(h_{i n t}+\sqrt{m_{c}^{\|} m_{c}^{\perp}}\right)\left(\rho / \rho_{i n t}\right)^{2 K}+\left(h_{i n t}-\sqrt{m_{c}^{\|} m_{c}^{\perp}}\right)}{\left(h_{i n t}+\sqrt{m_{c}^{\|} m_{c}^{\perp}}\right)\left(\rho / \rho_{i n t}\right)^{2 K}-\left(h_{i n t}-\sqrt{m_{c}^{\|} m_{c}^{\perp}}\right)}} & \rho_{i n t}<\rho \leq \rho_{c}, \\ m_{r} \frac{\left(h_{c}+m_{r}\right)\left(\rho / \rho_{c}\right)^{2}+\left(h_{c}-m_{r}\right)}{\left(h_{c}+m_{r}\right)\left(\rho / \rho_{c}\right)^{2}-\left(h_{c}-m_{r}\right)} & \rho_{c}<\rho \leq 1,\end{cases}
$$

donde $K=\sqrt{m_{c}^{\|} / m_{c}^{\perp}}, \mathrm{y}$

$$
\begin{aligned}
h_{i n t} & =h\left(\rho_{i n t}\right)=m_{i n t} \frac{f_{\text {int }} m_{i n t}+\left(f_{i n t}+2 f_{i n c}\right) m_{i n c}}{\left(f_{i n t}+2 f_{i n c}\right) m_{i n t}+f_{i n t} m_{i n c}}, \\
h_{c} & =h\left(\rho_{c}\right)=\sqrt{m_{c}^{\|} m_{c}^{\perp}} \frac{\left[f_{c}^{K}+\left(f_{i n t}+f_{i n c}\right)^{K}\right] h_{i n t}+\left[f_{c}^{K}-\left(f_{i n t}+f_{i n c}\right)^{K}\right] \sqrt{m_{c}^{\|} m_{c}^{\perp}}}{\left[f_{c}^{K}-\left(f_{i n t}+f_{i n c}\right)^{K}\right] h_{i n t}+\left[f_{c}^{K}+\left(f_{i n t}+f_{i n c}\right)^{K}\right] \sqrt{m_{c}^{\|} m_{c}^{\perp}}} .
\end{aligned}
$$

Aquí, $f_{c}, f_{\text {int }}$ y $f_{\text {inc }}$ indican las fracciones volumétricas del núcleo, la interfase y la inclusión, 
respectivamente. Por ende, en $\rho=1$ se tiene que $\left(\rho / \rho_{c}\right)^{2}=1 / f_{c}$ y por ende

$$
\bar{m}=h(1)=m_{r} \frac{\left(1+f_{c}\right) h_{c}+\left(1-f_{c}\right) m_{r}}{\left(1-f_{c}\right) h_{c}+\left(1+f_{c}\right) m_{r}} .
$$

Nuevamente, considerando que ahora las movilidades son las movilidades efectivas del laminado bifásico, retomando el uso del subíndice $\alpha$ que indica la especie iónica y reemplazando en (5.25), el resultado obtenido es

$$
\overline{\eta h}_{\alpha}=\not h_{\alpha}^{(a)} \frac{\left(1+f_{c}\right) A_{\alpha}+\left(1-f_{c}\right) \eta_{\alpha}^{(a)}}{\left(1-f_{c}\right) A_{\alpha}+\left(1+f_{c}\right) \eta_{\alpha}^{(a)}},
$$

donde

$$
\begin{aligned}
& A_{\alpha}=\sqrt{\widetilde{\not h}_{\alpha}^{\|} \widetilde{\not h}_{\alpha}^{\perp}} \frac{\left[f_{c}^{K}+\left(f^{(i n t)}+f^{(i n c)}\right)^{K}\right] B_{\alpha}+\left[f_{c}^{K}-\left(f^{(i n t)}+f^{(i n c)}\right)^{K}\right] \sqrt{\widetilde{\not h}_{\alpha}^{\|} \widetilde{\not h}_{\alpha}^{\perp}}}{\left[f_{c}^{K}-\left(f^{(i n t)}+f^{(i n c)}\right)^{K}\right] B_{\alpha}+\left[f_{c}^{K}+\left(f^{(i n t)}+f^{(i n c)}\right)^{K}\right] \sqrt{\widetilde{\not h}_{\alpha}^{\|} \widetilde{\not h}_{\alpha}^{\perp}}}, \\
& B_{\alpha}=\not h_{\alpha}^{(i n t)} \frac{f^{(i n t)}}{f^{(i n t)}+2 f^{(i n c)}} .
\end{aligned}
$$

En estas expresiones, $f^{(i n c)}$ y $f^{(i n t)}$ representan la fracción volumétrica de las inclusiones e interfases respectivamente, $f_{c}$ es la fracción volumétrica del núcleo compuesto por la inclusión, la interfase y la cilindrita, los tensores de movilidad ponderados quedan determinados por

$$
\not h_{\alpha}^{(r)}=\frac{c_{0 \alpha}^{(r)}}{f^{(c r)} c_{0 \alpha}^{(c r)}+f^{(a)} c_{0 \alpha}^{(a)}+f^{(i n t)} c_{0 \alpha}^{(i n t)}} m_{\alpha}^{(r)}
$$

$(r=a, c r, i n t), \mathrm{y} K=\sqrt{\widetilde{\not h}_{\alpha}^{\|} / \widetilde{\eta h}_{\alpha}^{\perp}}$. Bajo condiciones estacionarias, la conductividad efectiva está dada por (5.17) con $\overline{\eta 巾}_{+}$ahora determinada por (5.26).

Resultados numéricos, a modo de ejemplo, son presentados para el caso de $f_{c}^{c r}=1-$ $f_{c}^{a}=0,25, f_{r}=1-f_{c}=0,6\left(1-f^{(i n c)}-f^{(i n t)}\right), c_{0 \alpha}^{(c r)} / c_{0 \alpha}^{(a)}=1, m_{\alpha}^{\|} / m_{\alpha}^{\perp}=1000 \mathrm{y}\left(m_{\alpha}^{\|}+\right.$ $\left.m_{\alpha}^{\perp}\right) /\left(2 m_{\alpha}^{(a)}\right)=5$ para ambas especies iónicas, $\mathrm{y} m_{-}^{(a)}=3 m_{+}^{(a)}$. De esta manera, la movilidad de los aniones por fuera de la interfase es tres veces mayor que la correspondiente movilidad 
a)

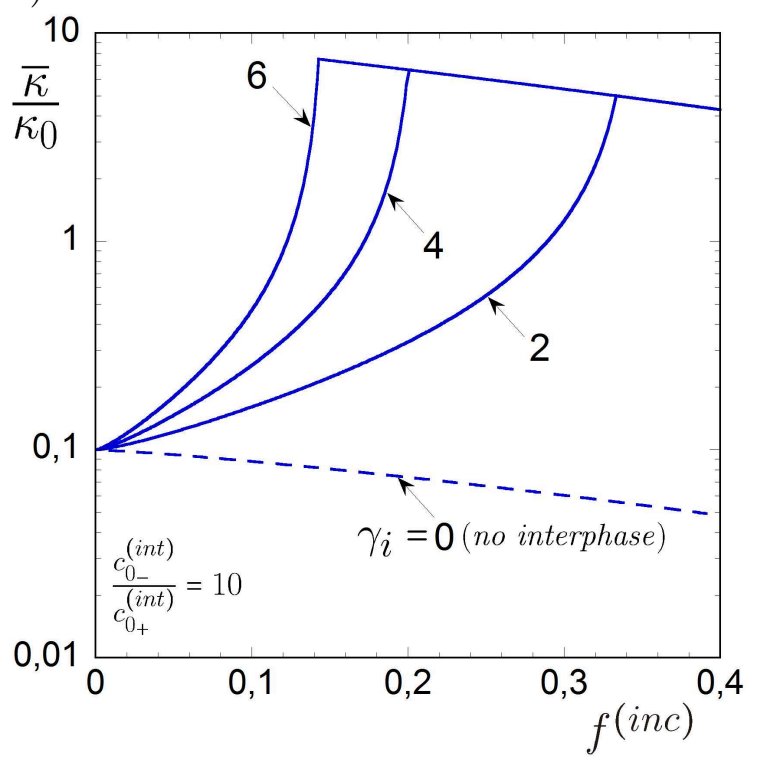

b)

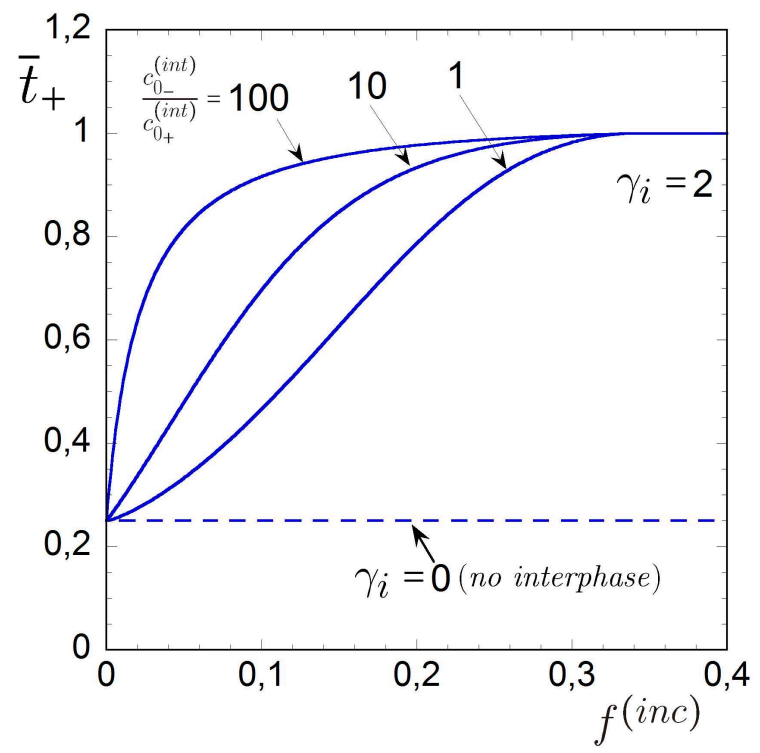

Figura 5.4: Propiedades de transporte de un electrolito compuesto (EO:Li=10) como función del contenido de partículas, para diferentes tasas de crecimiento de las interfases y diferentes relaciones de concentración $c_{0-}^{(i n t)} / c_{0+}^{(i n t)}$ : (a) conductividad efectiva, adimensionalizada con la conductividad de referencia $\kappa_{0}$; (b) número de transporte catiónico efectivo.

del catión, resultando en un número de transporte, en ausencia de partículas, de $\bar{t}^{+}=$ $1 /(1+3)=0,25$. Sin embargo, para reproducir el efecto de anclaje de los aniones en una interfase apropiadamente conductiva, se asume que $m_{-}^{(i n t)}=0,033 m_{-}^{(a)}$ y $m_{+}^{(i n t)}=100 m_{+}^{(a)}$, $c_{0+}^{(i n t)} / c_{0+}^{(a)}=1$, además de altos valores para la relación $c_{0-}^{(i n t)} / c_{0-}^{(a)}$ : una alta concentración de referencia $c_{0-}^{(i n t)}$ "atrae" aniones a la interfase mientras que una movilidad baja $m_{-}^{(i n t)}$ les impide escapar fácilmente de la misma. Adicionalmente, se ha considerado $\bar{c}_{+}=\left(1-f^{(i n c)}\right) c$ con $c / c_{0}=1 / 10$ (i.e., EO:Li=10:1), y se ha asumido que el tamaño de la interfase depende linealmente del tamaño de la inclusión, de acuerdo a

$$
f^{(i n t)}=\gamma_{i} f^{(i n c)}
$$

La Figura 5.4 muestra predicciones para la conductividad efectiva y el número de transporte del catión en función del contenido de partículas para diferentes tasas de crecimiento de la interfase $\gamma_{i}$ y relaciones de concentración de referencia $c_{0-}^{(i n t)} / c_{0-}^{(a)}$. En ausencia de interfase $\left(\gamma_{i}=0\right)$, el número de transporte del catión es independiente del contenido de partículas y la conductividad efectiva decrece moderadamente con el incremento de la cantidad de 
partículas, como resultado del efecto aislante de las inclusiones y del decrecimiento global de la densidad molar del litio. Por el contrario, en presencia de las interfases ambas propiedades varían fuertemente con la concentración de partículas. En el caso de la conductividad efectiva, las predicciones muestran que el incremento inicial puede ser de hasta dos órdenes de magnitud y alcanzar un máximo cuando las interfases percolan - la percolación de las interfases se da en $f^{(i n c)}=1 /\left(1+\gamma_{i}\right)$ para el caso de (5.30)-, véase la Figura 5.4-a. A partir de ese punto, el material modelo se transforma en un compuesto de dos fases, formado por una matriz isótropa conductiva y partículas aislantes; por lo tanto, la conductividad iónica decrece de forma monótona con el incremento del contenido de partículas. Estas tendencias son consistentes con datos experimentales disponibles en compuestos de PEO (Siekierski et al. 2007), que muestran un máximo en el rango de 0.05-0.3 para el contenido de partículas. Así, el modelo suguiere que la percolación de las interfases puede de hecho ser uno de los mecanismos detrás del máximo observado en dichos sistemas.

En el caso del número de transporte del catión, las predicciones también muestran un incremento con el aumento de contenido de partículas hasta el punto en el cual las interfases percolan. Más allá de la percolación, $\bar{t}_{+}$se mantiene constante y cercano a la unidad. De esta manera, una combinación de un alto $c_{0-}^{(i n t)}$ con un bajo $m_{-}^{(i n t)}$ aparentemente reproduce el efecto de "anclaje" de los aniones dentro de la región de la interfase. En particular, para

el caso de $c_{0-}^{(i n t)} / c_{0+}^{(i n t)}=10$ se reproduce un incremento del número de transporte del catión en presencia de un contenido de partículas comparable con los reportados para compuestos de PEO (Croce et al. 2001) y para otros electrolitos poliméricos compuestos (Stephan \& Nahm 2006). Esto deja en claro la importancia de contemplar la variación espacial de las concentraciones de referencia cuando se modelan electrolitos sólidos microestructurados.

\subsection{Observaciones finales}

En el problema modelo desarrolado anteriormente se han homogenizado las ecuaciones de campo para formas específicas de las funciones de energía $W^{(r)}$ y de los potenciales de disipación $U^{(r)}$, como fue detallado en (4.1)-(4.2). Dichas formas son propias de sólidos polarizables que contienen concentraciones diluidas de iones y que presentan comportamientos 
lineales en sus propiedades de transporte. Sin embargo, el proceso de homogenización puede ser aplicado a funciones de energía y de disipación más complejas que describan fenómenos adicionales como polarización no lineal e interacciones entre iones en concentraciones no diluidas. Dichos análisis no lineales son requeridos, por ejemplo, para estimar la influencia de los parámetros microestructurales sobre la resistencia dieléctrica de electrolitos sólidos, una propiedad de particular relevancia para el diseño de baterías. Dicha posibilidad fue también explorada en el marco de este trabajo y se presenta a continuación. 


\section{Capítulo 6}

\section{Transporte Iónico No Lineal}

\subsection{Respuesta Efectiva}

La respuesta efectiva de electrolitos microestructurados puede ser formalmente obtenida evaluando el transporte de iones a través de una secuencia de sistemas materiales con $\Omega$ fijo y donde la escala de longitud inferior de dicha secuencia sea decreciente. Ya se ha detallado, en el capítulo 4, la evaluación de dicho límite utilizando el método de convergencia multiescala de Allaire \& Briane (1996) para energías de polarización y potenciales de disipación cuadráticos. Si bien dicho análisis fue realizado de manera exclusiva para ese tipo de relaciones, es razonable suponer que la estructura resultante obtenida del sistema multi-escala pueda ser utilizada para energías y potenciales de disipasión convexos de manera general. Desafortunadamente no se dispone aún de una demostración matemática rigurosa que permita asegurarlo y es sólo de manera heurística que podemos basarnos en él. En este caso, hecha la aclaración previa, las ecuaciones de campo macroscópicas son escritas de la siguente manera

$$
\dot{\bar{c}}_{\alpha}+\nabla \cdot \overline{\mathbf{j}}_{\alpha}=0 \quad \text { y } \quad \nabla \cdot \overline{\mathbf{d}}=F \sum_{\alpha=1}^{A} z_{\alpha} \bar{c}_{\alpha}
$$


y las relaciones constitutivas efectivas son expresadas en términos de los potenciales efectivos como

$$
\overline{\mathbf{j}}_{\alpha}=-\bar{c}_{\alpha} \frac{\partial \widetilde{U}_{\alpha}}{\partial \nabla \bar{\mu}_{\alpha}}\left(\nabla \bar{\mu}_{\alpha}\right) \quad \text { y } \quad \overline{\mathbf{d}}=\frac{\partial \widetilde{W}_{e}}{\partial \overline{\mathbf{e}}}(\overline{\mathbf{e}})
$$

Aquí la barra superior indica los campos macroscópicos, luego los potenciales efectivos quedan dados por

$$
\widetilde{U}_{\alpha}(\overline{\mathbf{g}})=\min _{\mathbf{g} \in \mathcal{K}(\overline{\mathbf{g}})} \sum_{r=1}^{N} \theta^{(r)}\left\langle\hat{U}_{\alpha}^{(r)}(\mathbf{g})\right\rangle^{(r)} \quad \text { y } \quad \widetilde{W}_{e}(\overline{\mathbf{e}})=\min _{\mathbf{e} \in \mathcal{K}(\overline{\mathbf{e}})} \sum_{r=1}^{N} \theta^{(r)}\left\langle\hat{W}_{e}^{(r)}(\mathbf{e})\right\rangle^{(r)}
$$

donde los potenciales locales son expresados como

$$
\hat{U}_{\alpha}^{(r)}(\mathbf{g})=\frac{c_{0 \alpha}^{(r)}}{\left\langle c_{0 \alpha}\right\rangle} U_{\alpha}^{(r)}(\mathbf{g}) \quad \text { y } \quad \hat{W}_{e}^{(r)}(\mathbf{e})=\frac{\epsilon_{0}}{2} \mathbf{e} \cdot \mathbf{e}+W_{p}^{(r)^{*}}(\mathbf{e})
$$

En estas expresiones, $\langle\cdot\rangle$ y $\langle\cdot\rangle^{(r)}$ indican los promedios volumétricos sobre el elemento de volumen representativo del sólido heterogéneo y sobre el subdominio ocupado por la fase $r$, respectivamente, $\theta^{(r)}=\left\langle\chi^{(r)}\right\rangle$ es la fracción volumétrica de la fase $r, \mathcal{K}(\overline{\mathbf{a}})$ es el conjunto de campos de vectores gradientes con promedio volumétrico $\overline{\mathbf{a}}, \mathrm{y}(\cdot)^{*}$ indica la transformada de Legendre. Si los potenciales locales son funciones cuadráticas de sus argumentos, las expresiones anteriores se reducen a las derivadas en el capítulo 3.

Consecuentemente, la respuesta efectiva de electrolito queda completamente determinada por los potenciales efectivos (6.3). Téngase en cuenta que dichos potenciales están matemáticamente desacoplados y pueden por ende ser calculados de manera independiente. De aquí en adelante el foco estará puesto en estimar los potenciales de disipación $\widetilde{U}_{\alpha}$. Sin embargo, es importante enfatizar que la metodología descripta a continuación puede ser utilizada mutatis mutandis para estimar los potenciales efectivos $\widetilde{W}_{e}$ de manera análoga, dada su similar estructura matemática. 


\subsection{Estimaciones de Homogenización Lineal de Comparación}

Las estimaciones para los potenciales de disipación efectiva realizadas en este trabajo son realizadas por medio del Método de Homogenización Lineal de Comparación propuesto por Ponte Castañeda (2001). En esta sección se presentan las fórmulas relevantes para calcular las estimaciones lineales de comparación para compuestos conformados por fases isótropas, previemente se introduce el método original de la cita ya nombrada. En caso de requerir mayores detalles de dicha derivación es pertinente dirigirse al trabajo de Ponte Castañeda (2001).

\subsubsection{Método de Homogenización Lineal de Comparación}

Siguiendo el trabajo de Ponte Castañeda (2001) se introduce un "material lineal de comparación" con una función de densidad de energía $U_{0}$ dada por

$$
\hat{U}_{0}(\mathbf{x}, \mathbf{g})=\sum_{r=1}^{N} \chi^{(r)}(\mathbf{x}) U_{0}^{(r)}(\mathbf{g})
$$

Los potenciales lineales de comparación corresponden a expansiones de Taylor de segundo orden del potencial no lineal, de la forma

$$
\hat{U}_{0}^{(r)}(\mathbf{g})=\hat{U}^{(r)}\left(\breve{\mathbf{g}}^{(r)}\right)+\frac{\partial \hat{U}^{(r)}}{\partial \mathbf{g}}\left(\breve{\mathbf{g}}^{(r)}\right) \cdot\left(\mathbf{g}-\breve{\mathbf{g}}^{(r)}\right)+\frac{1}{2}\left(\mathbf{g}-\breve{\mathbf{g}}^{(r)}\right) \cdot \mathbf{M}_{0}^{(r)}\left(\mathbf{g}-\breve{\mathbf{g}}^{(r)}\right)
$$

donde los gradientes de referencia $\breve{\mathbf{g}}^{(r)}$ y las movilidades lineales $\mathbf{M}_{0}^{(r)}$ son propiedades uniformes en cada fase $r$ que deben ser especificadas. Dichos potenciales también pueden ser expresados de la siguiente manera

$$
\hat{U}_{0}^{(r)}(\mathbf{g})=\frac{1}{2} \mathbf{g} \cdot \mathbf{M}_{0}^{(r)} \mathbf{g}+\mathbf{J}_{0}^{(r)} \cdot \mathbf{g}+f_{0}^{(r)}
$$


donde

$$
\begin{aligned}
\mathbf{J}_{0}^{(r)} & =\frac{\partial \hat{U}^{(r)}}{\partial \mathbf{g}}\left(\breve{\mathbf{g}}^{(r)}\right)-\mathbf{M}_{0}^{(r)} \breve{\mathbf{g}}^{(r)} \mathrm{y} \\
f_{0}^{(r)} & =\hat{U}^{(r)}\left(\breve{\mathbf{g}}^{(r)}\right)-\frac{\partial \hat{U}^{(r)}}{\partial \mathbf{g}}\left(\breve{\mathbf{g}}^{(r)}\right) \cdot \breve{\mathbf{g}}^{(r)}+\frac{1}{2} \breve{\mathbf{g}}^{(r)} \cdot \mathbf{M}_{0}^{(r)} \breve{\mathbf{g}}^{(r)}
\end{aligned}
$$

Por lo tanto, el potencial efectivo del compuesto linear de comparación es

$$
\widetilde{U}_{0}(\overline{\mathbf{g}})=\min _{\mathbf{g} \in \mathcal{K}(\overline{\mathbf{g}})} \sum_{r=1}^{N} \theta^{(r)}\left\langle\frac{1}{2} \mathbf{g} \cdot \mathbf{M}_{0}^{(r)} \mathbf{g}+\mathbf{J}_{0}^{(r)} \cdot \mathbf{g}+f_{0}^{(r)}\right\rangle^{(r)}
$$

Se introducen luego las funciones de "error" $V^{(r)}$ tal que los potenciales de cada fase puedan expresarse como

$$
U^{(r)}(\mathbf{g})=U_{0}^{(r)}(\mathbf{g})+V^{(r)}\left(\mathbf{g}^{(r)}, \mathbf{M}_{0}^{(r)}\right)
$$

Dichas funciones de error pueden ser definidas de diversas formas, elegimos la forma original del trabajo citado donde

$$
V^{(r)}\left(\mathbf{g}^{(r)}, \mathbf{M}_{0}^{(r)}\right)=\underset{\hat{g}^{(r)}}{\operatorname{stat}}\left[U^{(r)}\left(\hat{g}^{(r)}\right)-U_{0}^{(r)}\left(\hat{g}^{(r)}\right)\right]
$$

Dicha operación de minimización, en una aplicación más amplia del concepto, se transforma en una operación de búsqueda de un punto estacionario, ya que puede tener varios mínimos locales. Además es importante remarcar que permite opciones diversas a la trivial $\hat{g}^{(r)}=$ $g^{(r)}$. La opción elegida en el método de la secante generalizada implica la condición de estacionaridad dada por

$$
\frac{\partial U^{(r)}}{\partial \mathbf{g}}\left(\hat{\mathbf{g}}^{(r)}\right)-\frac{\partial U^{(r)}}{\partial \mathbf{g}}\left(\mathbf{g}^{(r)}\right)=\mathbf{M}_{0}^{(r)}\left(\hat{\mathbf{g}}^{(r)}-\mathbf{g}^{(r)}\right),
$$

donde es importante remarcar que $\hat{\mathbf{g}}^{(r)}$ no necesariamente tiene que estar alineado con $\mathbf{g}^{(r)}$ y que el caso en el cual $\hat{\mathbf{g}}^{(r)} \neq \mathbf{g}^{(r)}$ y $\mathbf{g}^{(r)} \neq 0$ corresponde a la aproximación de la secante generalizada que, como se enfatiza en el trabajo original, difiere de las aproximaciones de la secante y la tangente, utilizada en otros trabajos anteriores al citado. El potencial efectivo 
queda finalmente de la siguiente manera

$$
\widetilde{U}(\overline{\mathbf{g}})=\underset{\hat{g}^{(r)}}{\operatorname{stat}} \sum_{r=1}^{N} \theta^{(r)}\left\langle U_{0}^{(r)}+V^{(r)}\left(\mathbf{g}^{(r)}, \mathbf{M}_{0}^{(r)}\right)\right\rangle^{(r)} .
$$

\subsubsection{Compuestos con fases constitutivas isótropas}

Consideramos aquí sistemas materiales cuyas fases consitutivas sean isótropas, tal que

$$
U_{\alpha}^{(r)}(\mathbf{g})=u_{\alpha}^{(r)}(|\mathbf{g}|) \quad \text { y } \quad \hat{U}_{\alpha}^{(r)}(\mathbf{g})=\hat{u}_{\alpha}^{(r)}(|\mathbf{g}|)
$$

Dado que los potenciales efectivos asociados con las diversas especies iónicas están matemáticamente desacoplados, desde aquí nos centraremos en una sola especie y se omitirá el subíndice $\alpha$ para simplificar la notación.

Los potenciales lineales de comparación, como ya se expresó en (6.6) corresponden a expansiones de Taylor de segundo orden del potencial no lineal. Y según el procedimiento ya detallado se puede llegar a la expresión (6.10) para el potencial efectivo del compuesto lineal de comparación. En el caso de fases isótorpas, las propiedades lineales de comparación óptimas $\breve{\mathbf{g}}^{(r)}$ y $\mathbf{M}_{0}^{(r)}$ están dadas por

$$
\breve{\mathbf{g}}^{(r)}=\overline{\mathbf{g}}^{(r)} \quad \mathrm{y} \quad \mathbf{M}_{0}^{(r)}=M_{\|}^{(r)} \frac{\overline{\mathbf{g}}^{(r)}}{\bar{g}^{(r)}} \otimes \frac{\overline{\mathbf{g}}^{(r)}}{\bar{g}^{(r)}}+M_{\perp}^{(r)}\left[\mathbf{I}-\frac{\overline{\mathbf{g}}^{(r)}}{\bar{g}^{(r)}} \otimes \frac{\overline{\mathbf{g}}^{(r)}}{\bar{g}^{(r)}}\right]
$$

donde $\overline{\mathbf{g}}^{(r)}$ es el promedio de $\mathbf{g}(\mathbf{x})$ en la fase $r$ en el compuesto lineal de comparación (6.10), $\bar{g}^{(r)}=\left|\overline{\mathbf{g}}^{(r)}\right|$, y las movilidades corresponden a linealizaciones del potencial no lineal realizadas por medio de la secante generalizada, dadas por

$$
\hat{u}^{(r)^{\prime}}\left(\hat{g}^{(r)}\right) \frac{\hat{g}_{\|}^{(r)}}{\hat{g}^{(r)}}-\hat{u}^{(r)^{\prime}}\left(\bar{g}^{(r)}\right)=M_{\|}^{(r)}\left(\hat{g}_{\|}^{(r)}-\bar{g}^{(r)}\right) \quad \text { y } \quad \frac{\hat{u}^{(r)^{\prime}}\left(\hat{g}^{(r)}\right)}{\hat{g}^{(r)}}=M_{\perp}^{(r)}
$$

con $\hat{g}^{(r)}=\sqrt{\left(\hat{g}_{\|}^{(r)}\right)^{2}+\left(\hat{g}_{\perp}^{(r)}\right)^{2}}$ y la tilde indica la derivada. En estas expresiones, las variables "sombrero" están relacionadas con el primer y segundo momento del campo gradiente $\mathbf{g}(\mathbf{x})$ 
en el compuesto lineal de comparación (6.10) via

$$
\hat{g}_{\|}^{(r)}=\bar{g}^{(r)}+\sqrt{C_{\|}^{(r)}} \quad \text { y } \quad \hat{g}_{\perp}^{(r)}=\sqrt{C_{\perp}^{(r)}}
$$

donde

$$
\overline{\mathbf{g}}^{(r)}=\frac{1}{\theta^{(r)}} \frac{\partial\left(\widetilde{U}_{0}-\bar{f}\right)}{\partial \mathbf{J}_{0}^{(r)}} \quad \text { y } \quad C_{\|, \perp}^{(r)}=\frac{2}{\theta^{(r)}} \frac{\partial \widetilde{U}_{0}}{\partial M_{\|, \perp}^{(r)}}
$$

Aquí, $\bar{f}=\sum_{r=1}^{N} \theta^{(r)} f^{(r)}$, y en la primera expresión la derivada es realizada manteniendo $\mathbf{M}_{0}^{(r)}$ constante mientras que en la segunda expresión se mantiene fijo $\breve{\mathbf{g}}^{(r)}$. Las cantidades $(6.19)_{2}$ corresponden a las trazas del tensor de covarianzas $\mathbf{C}_{\mathbf{g}}^{(r)}=\left\langle\left(\mathbf{g}(\mathbf{x})-\overline{\mathbf{g}}^{(r)}\right) \otimes\left(\mathbf{g}(\mathbf{x})-\overline{\mathbf{g}}^{(r)}\right)\right\rangle^{(r)}$ dentro de cada fase $r$. Por lo tanto, el esquema de linealización depende no sólo de los primeros momentos de los campos sino de las fluctuaciones interfásicas. Dada una estimación lineal para el potencial efectivo de comparación (6.10), las expresiones (6.16) a (6.19) consituyen un sistema de ecuaciones algebraicas no lineales para las cantidades $\hat{g}_{\|, \perp}^{(r)}$ y $M_{\|, \perp}^{(r)}, r=1, \ldots, N$.

Finalmente la estimación homogenizada del potencial efectivo, utilizando la secante generalizada, queda dada por

$$
\widetilde{U}(\overline{\mathbf{g}})=\sum_{r=1}^{N} \theta^{(r)}\left[\hat{u}^{(r)}\left(\hat{g}^{(r)}\right)-\hat{u}^{(r)^{\prime}}\left(\bar{g}^{(r)}\right)\left(\hat{g}_{\|}^{(r)}-\bar{g}^{(r)}\right)\right]
$$

En el capítulo siguiente se considerarán formas específicas de las funciones potenciales de modo de obtener resultados para un caso de interés académico.

\subsubsection{Estimaciones duales}

Las relaciones constitutivas (6.2) pueden ser invertidas de manera que los gradientes del potencial electroquímico sean expresados en términos de los flujos molares. Dicha inversión se realiza a través de las expresiones duales de Legendre de los potenciales de disipación. La técnica de comparación lineal de la Sección 6.2.2 puede ser utilizada de manera completamente análoga para derivar estimaciones para las relaciones constitutivas directamente a 
partir del problema dual. Las expresiones no son incluídas en este trabajo, sin embargo se aclara que, como fue detallado por Ponte Castañeda (2001), las estimaciones obtenidas de la formulación primaria y su dual no son generalmente expresiones de Legendre duales entre sí, es decir no son equivalentes, debido a ciertas aproximaciones realizadas en la optimización del medio lineal de comparación. Sin embargo, los resultados presentados aquí arrojan que el duality-gap es muy pequeño e incluso desaparece en casos de fuertes no linealidades. 


\section{Capítulo 7}

\section{Problemas Modelos No Lineales}

\subsection{Materiales de dos fases con disipación tipo ley de la potencia}

Aquí se particularizan las fórmulas previamente presentadas para materiales de dos fases caracterizadas por potenciales del tipo ley de la potencia, es decir

$$
u^{(r)}(g)=\frac{G_{0}^{(r)}}{1+m}\left|\frac{g}{G_{0}^{(r)}}\right|^{1+m}
$$

donde el exponente $m$ es el mismo para todas las fases. Para el caso de $m=1$ dicho potencial corresponde al caso de respuesta lineal con movilidad iónica $\left(G_{0}^{(r)}\right)^{-1}$, mientras que $m \rightarrow \infty$ corresponde al caso de respuestas fuertemente no lineales en las que la corriente eléctrica es cero si el potencial del gradiente electroquímico tiene magnitudes por debajo del umbral $G_{0}^{(r)}$. De esta manera, dichos potenciales podrían ser usados para describir aproximadamente la respuesta de electrolitos sólidos poliméricos bajo un amplio rango de intensidades de campo eléctrico.

Por simplicidad, se restringe el análisis a compuestos de dos fases que presentan una simetría isótropa global. Consecuentemente, el potencial efectivo debe ser de la forma

$$
\widetilde{U}(\overline{\mathbf{g}})=\frac{\widetilde{G}_{0}}{1+m}\left|\frac{\overline{\mathbf{g}}}{\widetilde{G}_{0}}\right|^{1+m},
$$


donde $m$ es el mismo exponente que el de las fases constitutivas y $\widetilde{G}_{0}$ es una propiedad efectiva que depende de las propiedades locales $G_{0}^{(r)}$, de la microestructura y del exponente $m$.

Así, la estimación lineal de comparación (6.20) arroja una estimación para $\widetilde{G}_{0}$. Para el caso especial de compuestos bifásicos, la expresión (6.10) puede ser simplificada en virtud de las llamadas relaciones de Levin.

$$
\widetilde{U}_{0}(\overline{\mathbf{g}})=\bar{f}_{0}+\bar{J}_{0} \cdot \overline{\mathbf{g}}+\frac{1}{2} \overline{\mathbf{g}} \cdot \overline{\mathbf{M}}_{0} \overline{\mathbf{g}}+\frac{1}{2}\left[\overline{\mathbf{g}}+\left(\Delta \mathbf{M}_{0}\right)^{-1} \Delta \mathbf{J}_{0}\right] \cdot\left(\widetilde{\mathbf{M}}_{0}-\overline{\mathbf{M}}_{0}\right)\left[\overline{\mathbf{g}}+\left(\Delta \mathbf{M}_{0}\right)^{-1} \Delta \mathbf{J}_{0}\right]
$$

donde $\Delta \mathbf{M}_{0}=\mathbf{M}_{0}^{(2)}-\mathbf{M}_{0}^{(1)}$ y $\Delta \mathbf{J}_{0}=\mathbf{J}_{0}^{(2)}-\mathbf{J}_{0}^{(1)}$, y donde $\widetilde{\mathbf{M}}_{0}$ es el tensor de movilidad efectiva del sólido lineal. De esta manera, este potencial lineal de comparación queda completamente especificado en términos de $\widetilde{\mathbf{M}}_{0}$. Por lo tanto, haciendo uso de cualquier estimación de homogenización lineal para $\widetilde{\mathbf{M}}_{0}$, se pueden obtener estimaciones correspondientes para el potencial no lineal. En este trabajo se ha hecho uso de las estimaciones MGA y EMA, que pueden ser generadas a partir de las siguientes expresiones de Willis (1977):

$$
\widetilde{\mathbf{M}}_{0}=\left\{\sum_{r=1}^{2} \theta^{(r)} \mathbf{M}_{0}^{(r)}\left[\mathbf{I}+\mathbf{T}^{(0)}\left(\mathbf{M}_{0}^{(r)}-\mathbf{M}^{(0)}\right)\right]^{-1}\right\}\left\{\sum_{s=1}^{2} \theta^{(s)}\left[\mathbf{I}+\mathbf{T}^{(0)}\left(\mathbf{M}_{0}^{(s)}-\mathbf{M}^{(0)}\right)\right]^{-1}\right\}^{-1}
$$

donde $\mathbf{M}^{(0)}$ indica la movilidad de un material homogéneo de referencia y $\mathbf{T}^{(0)}$ es un tensor de segundo orden que depende de $\mathbf{M}^{(0)}$ y de las funciones de correlacion de dos puntos de la microestructura. Definiendo el tensor de referencia $\mathbf{M}^{(0)}$ igual a $\mathbf{M}_{0}^{(1)}\left(\mathbf{M}_{0}^{(2)}\right)$ se obtienen las estimaciones MGA, apropiadas para microestructuras del tipo matriz-partícula con la fase $r=1(r=2)$ tomando el rol de matriz. Por otra parte, definiendo $\mathbf{M}^{(0)}$ igual a $\widetilde{\mathbf{M}}_{0}$ se obtienen estimaciones EMA, apropiadas para microestructuras granulares sin una fase contínua que juege el papel de una matriz.

En vistas de la isotropía global asumida, los promedios de las fases $\overline{\mathbf{g}}^{(r)}$ están alineados con el campo total $\overline{\mathbf{g}}$. Luego, los vectores unitarios $\overline{\mathbf{g}}^{(r)} / \bar{g}^{(r)}=\overline{\mathbf{g}} / \bar{g}$ son los mismos para todas las fases y los tensores de movilidad $(6.16)_{2}$ en el compuesto lineal de comparación 
están alineados. Además, si el tensor $\mathbf{M}^{(0)}$ es de la forma $(6.16)_{2}$, el tensor $\mathbf{T}^{(0)}$ es también del mismo tipo, con las componentes "paralelas" y "perpendiculares" dadas por (ej., Ponte Castañeda 2001)

$$
T_{\|}^{(0)}=k \frac{1-\alpha(k)}{(k-1) M_{\|}^{(0)}} \quad \mathrm{y} \quad T_{\perp}^{(0)}=\frac{k \alpha(k)-1}{2(k-1) M_{\perp}^{(0)}} \quad \text { con } \quad \alpha(k)=\frac{\arcsin \sqrt{(k-1) / k}}{\sqrt{k-1}},
$$

donde $k=M_{\|}^{(0)} / M_{\perp}^{(0)}$ es la relación de anisotropía del tensor $\mathbf{M}^{(0)}$. Notar que en (7.5) la relación de anisotropía fue asumida tal que $k>1$, pero las expresiones correspondientes para el caso de $k<1$ pueden ser obtenidas por continuación analítica. Las expresiones (7.3) a (7.5) especifican de manera completa el potencial efectivo del compuesto lineal de comparación.

En virtud de la expresión (6.20), la estimación lineal de comparación para la propiedad efectiva $\widetilde{G}_{0}$ puede ser finalmente calculada utilizando

$$
\widetilde{G}_{0}^{-m}=\sum_{r=1}^{2} \theta^{(r)} \frac{c_{0}^{(r)}}{\left\langle c_{0}\right\rangle}\left(G_{0}^{(r)}\right)^{-m}\left\{\left(\frac{\hat{g}^{(r)}}{\bar{g}}\right)^{m+1}-(m+1)\left(\frac{\bar{g}^{(r)}}{\bar{g}}\right)^{m}\left(\frac{\hat{g}_{\|}^{(r)}}{\bar{g}}-\frac{\bar{g}^{(r)}}{\bar{g}}\right)\right\} .
$$

donde $\left\langle c_{0}\right\rangle=\theta^{(1)} c_{0}^{(1)}+\theta^{(2)} c_{0}^{(2)}$.

\subsection{Resultados para sistemas de tipo matriz-inclusión}

En esta sección se reportan resultados, a modo de ejemplo, para sólidos de dos fases con comportamiento del tipo "ley de la potencia" obtenidos utilizando aproximaciones MaxwellGarnett (MGA) — también conocidas como aproximaciones Claussius-Mossotti- para estimar el potencial efectivo de los compuestos lineales de comparación. Es sabido que dicha aproximación es apropiada para materiales del tipo matriz-partícula con concentración de inclusiones entre baja y moderada (ej., Milton, 2001). A partir de aquí, las fases que toman el rol de la matriz y de la inclusión serán identificadas con $r=1$ y $r=2$, respectivamente. El interés aquí está puesto en el desempeño de la estimaciones lineales de comparación, anteriormente nombradas, en el contexto de fuertes no linealidades y contrastes en la heterogeneidad. Por ello se restringe el análisis a sistemas materiales con relaciones molares de 
referencia uniformes $\left(c_{0}^{(1)}=c_{0}^{(2)}\right)$ a modo de simplificar la descripción, por ende $u^{(r)}=\hat{u}^{(r)}$.

Estimaciones del tipo MGA provienen de fijar $\mathbf{M}^{(0)}=\mathbf{M}_{0}^{(1)}$ en (7.4). Ahora bien, esta aproximación implica campos uniformes dentro de la fase de la incusión del sólido lineal de comparación, debido a eso

$$
\begin{aligned}
\hat{\mathbf{g}}^{(2)} & =\overline{\mathbf{g}}^{(2)} \mathrm{y} \\
\mathbf{M}_{0}^{(2)} & =\frac{\partial^{2} \hat{u}^{(2)}}{\partial \mathbf{g} \partial \mathbf{g}}\left(\overline{\mathbf{g}}^{(2)}\right)=\frac{1}{G_{0}^{(2)}}\left|\frac{\bar{g}^{(2)}}{G_{0}^{(2)}}\right|^{m-1}\left[m \frac{\mathbf{g}}{\bar{g}} \otimes \frac{\overline{\mathbf{g}}}{\bar{g}}+\left(\mathbf{I}-\frac{\overline{\mathbf{g}}}{\bar{g}} \otimes \frac{\overline{\mathbf{g}}}{\bar{g}}\right)\right] .
\end{aligned}
$$

Por otra parte, para un material de dos fases se tiene que $\overline{\mathbf{g}}^{(2)}=\left(\overline{\mathbf{g}}-\theta^{(1)} \overline{\mathbf{g}}^{(1)}\right) / \theta^{(2)}$, y por ende el cálculo de las estimaciones lineales de comparación pueden ser reducidas a un sistema de tres ecuaciones algebraicas, no lineales, para las variables $\hat{g}_{\|}^{(1)}, \hat{g}_{\perp}^{(1)}$ and $\bar{g}^{(1)}$, que deben ser resueltas, en general, numéricamente.

Las nuevas estimaciones del tipo MGA, llamadas Estimaciones de la Secante Generalizada (GSEC) son comparadas más adelante con las cotas elementales de Weiner y las estimaciones Secantes (SEC) del tipo MGA de Ponte Castañeda (1992). Vale resaltar que las estimaciones SEC proveen una cota superior estricta para las estimaciones GSEC. Además están incluídas las simulaciones numéricas de Barrett \& Talbot (1996) para compuestos de esferas sometidas a un potencial afín o a una densidad de corriente uniforme en su límite exterior. Estas simulaciones arrojan límites superiores e inferiores $\left(\mathrm{BT}^{+} \mathrm{y} \mathrm{BT}^{-}\right)$para la resistividad global de una clase particular de compuestos conocidos como ensamble de esferas compuestas; cuando las fases son lineales, dichas simulaciones arrojan resultados exactos. La Figura 7.1-a, -b muestran varias predicciones para la resistividad efectiva $\widetilde{G}_{0}$, normalizada con la resistividad de la matriz $G_{0}^{(1)}$, como función del índice de no linealidad $m \geq 1$, para el caso de $\theta^{(1)}=$ $\theta^{(2)}=0,5 \mathrm{y}$ dos valores de contreaste en la resistividad. Inicialmente se debe notar que para materiales lineales las estimaciones SEC y GSEC concuerdan completamente con las estimaciones lineales MGA, en las cuales ambas están basadas. Estas estimaciones lineales son conocidas por ser alcanzadas por compuestos isótropos con ensambles de esferas y es por ello que los resultados de BT también concuerdan con dichas estimaciones en ese caso. Para materiales no lineales, los diversos métodos arrojan ciertas diferencias en las predicciones, si 
a)

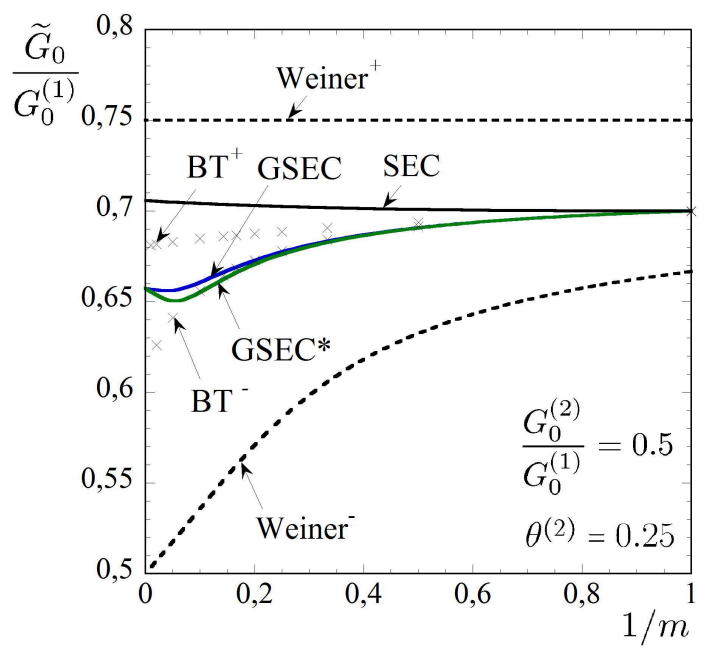

c)

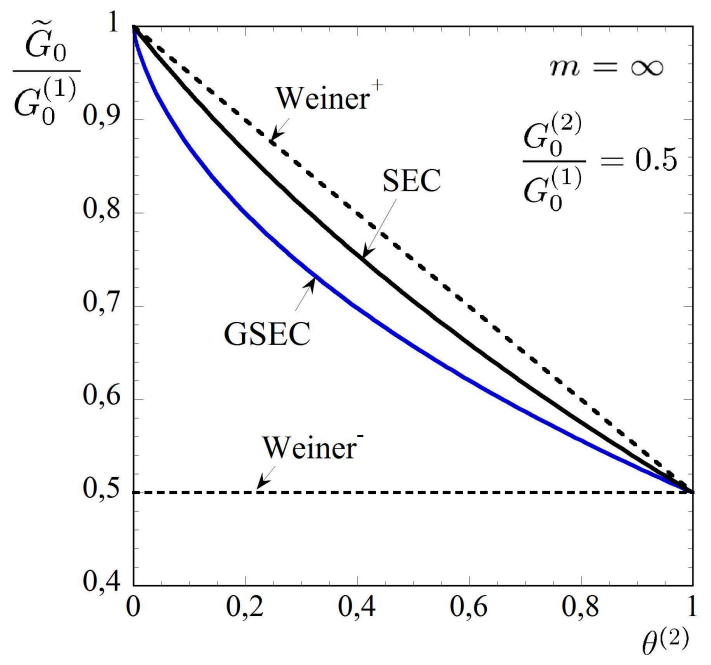

b)

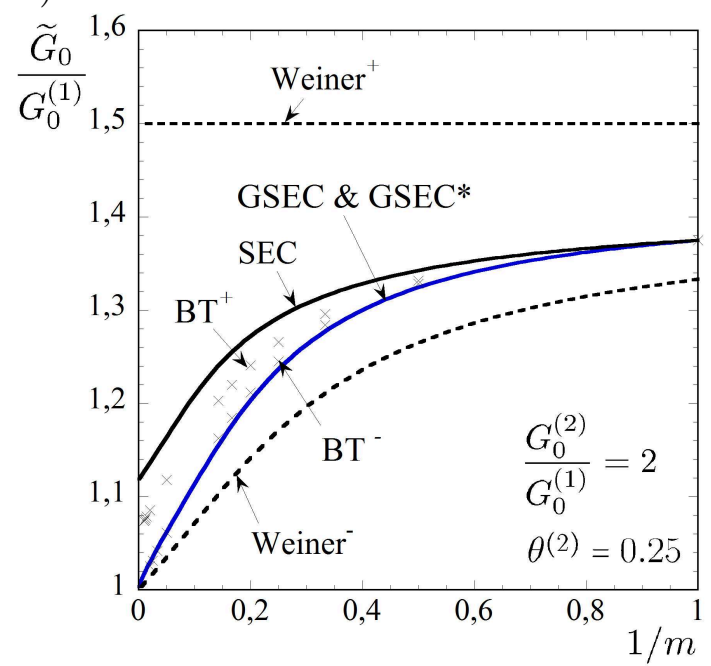

d)

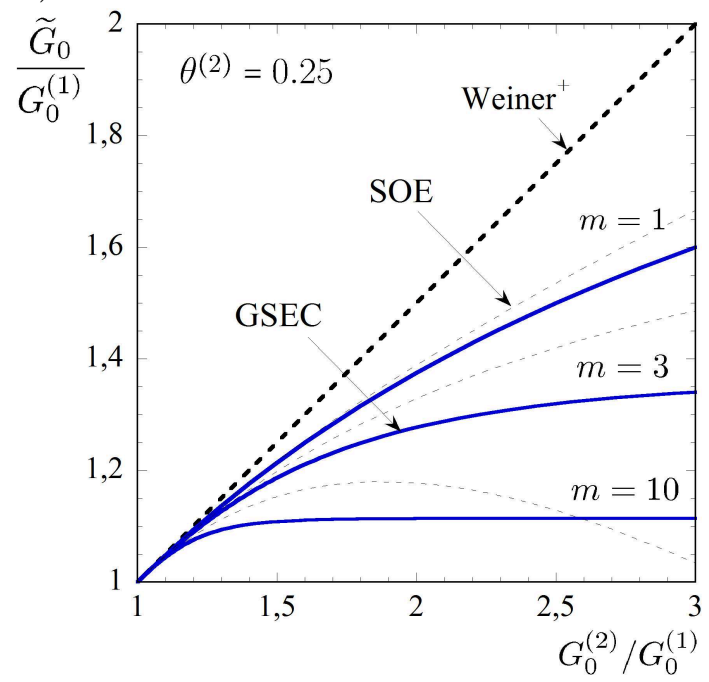

Figura 7.1: Estimaciones del tipo MGA para la resistividad efectiva de un compuesto de dos fases con no linealidad material $(m)$, concentración de inclusiones $\left(\theta^{(2)}\right)$ y relación de resistividades $\left(G_{0}^{(2)} / G_{0}^{(1)}\right)$.

bien se corrobora que las estimaciones GSEC son las que satisfacen todos los límites excepto el límite inferior $\mathrm{BT}^{-}$en el caso de inclusiones resistivas. Sin embargo, es importante notar que la diferencia es realmente pequeña y que los límites $\mathrm{BT}^{-}$demandan cálculos complejos que pueden haber introducido cierto error numérico. Como ya fue discutido en la sección 6.2.3, las estimaciones basadas en la secante generalizada presentan un duality-gap. Las estimaciones duales (GSEC*) son presentadas en las Figuras 7.1-a y -b para estimar dichas diferencias. La diferencia es muy pequeña en general y desaparece, no sólo en el caso lineal sino también en el 
caso límite de $m \rightarrow \infty$. Por lo tanto, ambas formulaciones generan predicciones equivalentes en la práctica.

El caso límite de no linealidad $m \rightarrow \infty$ corresponde a un compuesto conformado por fases de diferentes umbrales $G_{0}^{(r)}$ del gradiente de potencial electroquímico, siendo que el compuesto propiamente dicho posee un umbral efectivo $\widetilde{G}_{0}$. En dicho límite, los potenciales del tipo ley de la potencia (7.1) dejan de ser estrictamente convexos, el carácter de las ecuaciones que gobiernan el fenómeno cambia y aparece la posibilidad de que se establezcan campos de densidad de corriente discontinuos. De hecho, los resultados de Donev et al. (2002) y Duxbury et al. (2006) muestran que cuando el campo macroscópico alcanza el umbral efectivo el flujo de corriente se localiza en caminos, la cual es la solución al problema llamado "del camino más corto".

Para compuestos de partículas más resistivas $\left(G_{0}^{(2)}>G_{0}^{(1)}\right)$, las estimaciones GSEC no predicen un efecto en el umbral efectivo debido a la adición de inclusiones, es decir,

$$
\frac{\widetilde{G}_{0}}{G_{0}^{(1)}}=1
$$

independientemente del contraste y de la concentración de inclusiones. De acuerdo con Duxbury et al. (2006), en este caso el camino de la corriente intenta evitar las inclusiones pero al mismo tiempo trata de mantener su dirección lo más recta posible. Las estimaciones GSEC son consistentes con un camino totalmente recto. Por otra parte, los límites SEC y BT ${ }^{+}$ predicen la aparición de un efecto y por ende son consistentes con densidades de corriente que sean o bien difusas o bien localizadas a lo largo de líneas curvas en lugar de rectas. El hecho que el límite $\mathrm{BT}^{+}$sea obtenido considerando densidades de corriente uniformes en el borde exterior de la esfera compuesta le impide capturar la presencia de campos de corriente localizados.

Por otra parte, para compuestos con inclusiones menos resistivas $\left(G_{0}^{(2)}<G_{0}^{(1)}\right)$, las estimaciones GSEC sí predicen un efecto en el umbral efectivo debido al agregado de partículas. Se puede demostrar que dichas estimaciones se reducen a

$$
\frac{\widetilde{G}_{0}}{G_{0}^{(1)}}=\frac{1-\theta^{(2)}}{\sqrt{2 \theta^{(2)}}} \frac{1}{k[k \alpha(k)-1]} \frac{f_{\|}(k)+k f_{\perp}(k)}{\sqrt{f_{\|}(k)+f_{\perp}(k)}}+\frac{G_{0}^{(2)}}{G_{0}^{(1)}}
$$


donde las funciones $f_{\|}$y $f_{\perp}$ están dadas por

$$
f_{\|}(k)=k[1+k \alpha(k)(1-2 \alpha(k))] \quad \mathrm{y} \quad f_{\perp}(k)=(2+k) \alpha(k)-3,
$$

y la relación de anisotropía $k>1$ es solución a la ecuación no lineal

$$
1-\alpha(k)=\sqrt{\frac{\theta^{(2)}}{2}}\left(\frac{k-1}{k} \sqrt{f_{\|}(k)}-\frac{G_{0}^{(2)}}{G_{0}^{(1)}} \sqrt{f_{\|}(k)+f_{\perp}(k)}\right) .
$$

De acuerdo con Duxbury et al. (2006), en este caso el camino de la corriente busca la inclusión pero al mismo tiempo trata de mantener su dirección lo más recta posible. Consecuentemente, la inclusión interactúa aún cuando su fracción volumétrica es infinitesimalmente pequeña. Esto es esperable que se traduzca en una expansión no analítica del umbral efectivo para casos diluidos. Esto es exactamente lo que las estiamciones GSEC predicen. La Figura 7.1-c muestra estimaciones GSEC para el caso de $G_{0}^{(2)} / G_{0}^{(1)}=0,5$ como función de la concentración de inclusiones $\theta^{(2)}$, junto con los correspondientes límites de SEC y Weiner. De hecho, se puede observar que mientras los límites exhiben una pendiente lineal en $\theta^{(2)}=0$, las estimaciones GSEC presentan una pendiente infinita. Puede demostrarse que la expansión diluida de (7.10) es

$$
\frac{\widetilde{G}_{0}}{G_{0}^{(1)}}=1-\frac{3}{2}\left(1-\frac{G_{0}^{(2)}}{G_{0}^{(1)}}\right)^{4 / 3}\left(\frac{\pi}{2}\right)^{2 / 3}\left(\frac{\theta^{(2)}}{2}\right)^{2 / 3}
$$

que es no-analítica en $\theta^{(2)}=0$. Esta "singularidad del caso diluido" también fue observada en la rotura de dieléctricos de medios discretos (Duxbury et al. 1995) y continuos (Duxbury et al. 1990), así como en la fractura dúctil de medios porosos (Roux \& François 1991). Una expansión diluida similar con exponente de 2/3 fue encontrada para compuestos dieléctricos bi-dimensionales (Ponte Castañeda 2001). Sin embargo, en compuestos periódicos, aparecen diversas singularidades. Considérese un arreglo cúbico de inclusiones esfericas embebidas en una matriz cuyo umbral es superior. En ese caso, el "camino más corto" corresponde a una línea recta pasando a través de las inclusiones. Es sencillo ver que dichos campos implican que $1-\widetilde{G}_{0} / G_{0}^{(1)} \sim\left(\theta^{(2)}\right)^{1 / 3}$ — véase Idiart et al. (2009) para un análisis similar en sólidos viscoplásticos bi-dimensionales. Una comparación con la expresión (7.13) siguiere 
que la aleatoriedad debilita la singularidad del límite diluido. En todo caso, es importante remarcar el hecho que las estimaciones de la secante generalizada son capaces de capturar dichos efectos que aparecen ante fuertes no linealidades.

Finalmente, en la Figura 7.1-d las estimaciones GSEC son presentadas como función del contraste en la heterogeneidad, con $G_{0}^{(2)} \geq G_{0}^{(1)}$, para diversos valores del índice de no linealidad $(m=1,3,10, \infty)$. Dichas estimaciones son comparadas con el límite superior de Weiner, que es insensible a $m$, y la expansión asintótica exacta de segundo orden (SOE) de Blumenfeld \& Bergman (1991). Se debe notar que el rango de validez de dichas expresiones se reduce a medida que $m \rightarrow \infty$, donde las estimaciones GSEC, también exactas en segundo orden en el contraste, no se ven degeneradas. También puede observarse que a medida que la no linealidad se incrementa, las estimaciones GSEC tienden a saturarse cada vez para menores contrastes. Por ende, las no linealidades aplifican el efecto del contraste en la heterogeneidad.

\subsection{Resultados para sistemas de tipo granulares}

En esta sección se reportan resultados, a modo de ejemplo, para sólidos de dos fases con comportamiento del tipo "ley de la potencia" donde fue utilizado el método de Aproximación del Medio Efectivo (EMA) de Bruggemann (1935) para estimar el potencial efectivo del compuesto lineal de comparación. Dicha aproximación es apropiada para microestructuras granulares (e.g., Milton, 2001). Las estimaciones del tipo EMA se obtienen fijando $\mathbf{M}^{(0)}=\widetilde{\mathbf{M}}_{0}$ en la expresión (7.4). Contrariamente a la aproximación MGA, este caso no fija campos uniformes en ninguna de las fases. Por ello, la expresión (7.4) deja de ser explícita para $\widetilde{\mathbf{M}}_{0}$, conllevando a dos ecuaciones no lineales para las movilidades efectivas $\widetilde{M}_{\|, \perp}$, además de las cinco ecuaciones para las variables $\hat{g}_{\|, \perp}^{(r)}$ y $\bar{g}^{(1)}$. Dicho sistema completo de ecuaciones no lineales generalmente debe ser resuelto de manera numérica.

A continuación las nuevas estimaciones de la secante generalizada (GSEC) del tipo EMA son comparadas con los límites elementales de Weiner y con las estimaciones secantes (SEC) del tipo EMA planteadas por Ponte Castañeda (1992). Cabe recordar que las estimaciones SEC proveen una cota superior estricta para las estimaciones GSEC correspondientes. Las Figuras 7.2-a y -b muestran varias predicciones para la resisitivdad efectiva $\widetilde{G}_{0}$, normalizada 
a)

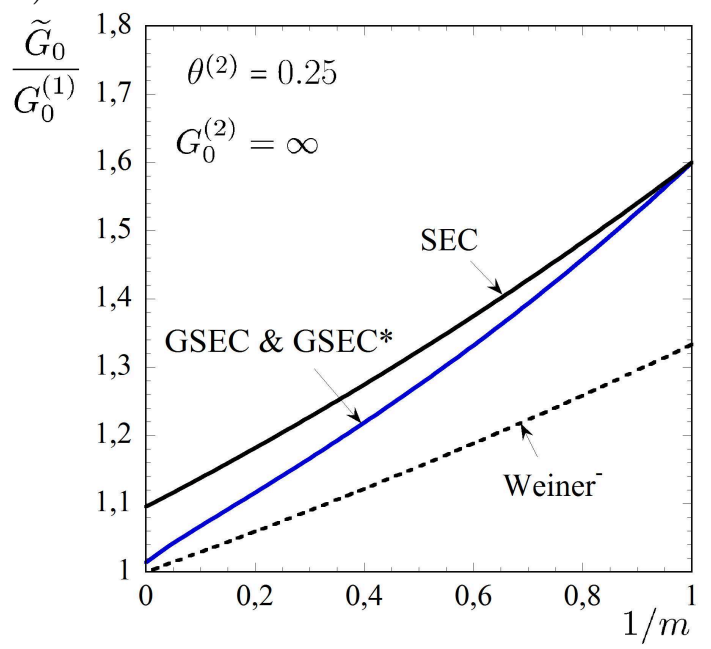

c)

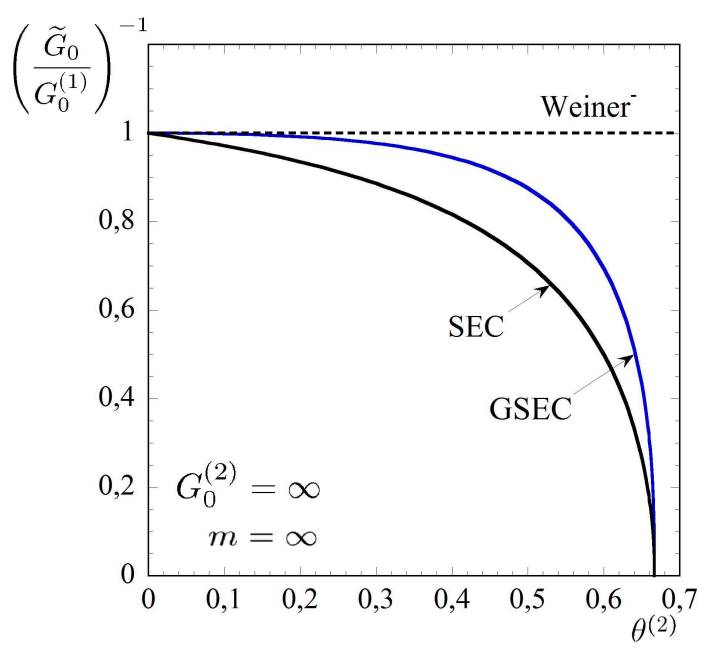

b)

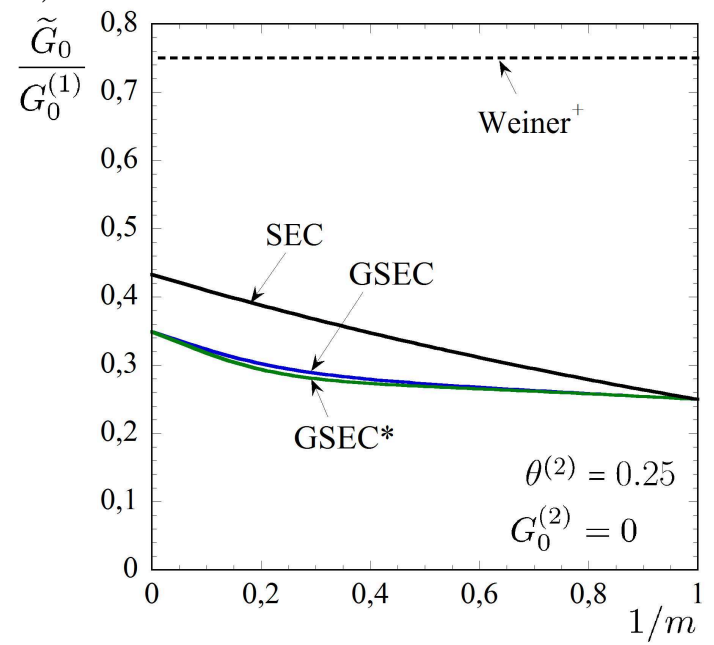

d)

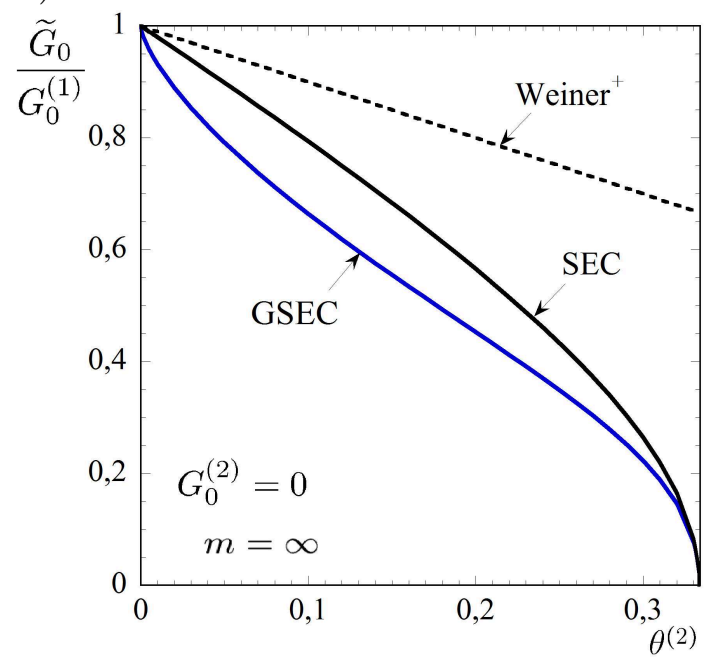

Figura 7.2: Estimaciones del método EMA para la resistividad efectiva de un compuesto bifásico en función de la no linealidad material $(m)$ y de la concentración de inclusiones $\left(\theta^{(2)}\right)$.

con la resistividad $G_{0}^{(1)}$, como función del índice de no linealidad $m \geq 1$, para $\theta^{(2)}=0,25$ y para los dos valores extremos del contraste en la resistividad. En el caso lineal las estimaciones SEC y GSEC concuerdan completamente con las estimaciones lineales EMA, en la cuales están basadas. Sin embargo, en el caso no lineal dichas estimaciones arrojan diferentes predicciones con las estimaciones GSEC siempre por debajo de las SEC, como es esperable. Una comparación con los resultados previos de la Figura 7.1 muestra que, contrariamente a las cotas de Weiner, las estimaciones de comparación lineal son capaces de capturar cierta interacción entre la microestructura y la alinealidad material. Se presentan también las estimaciones duales de la secante generalizada (GSEC*) para evaluar el tamaño del duality- 
gap. Una vez más la diferencia encontrada es en general muy pequeña y las predicciones convergen al mismo valor para el caso de $m \rightarrow \infty$ solamente cuando $G_{0}^{(2)}=0$. Cuando $G_{0}^{(2)}=\infty$ el duality-gap persiste en el límite, si bien es tan pequeño que virtualmente pueden ser consideradas iguales incluso en ese caso.

De hecho, contrariamente a las estimaciones GSEC basadas en la aproximación MGA, las estimaciones basadas en la aproximación EMA para $m \rightarrow \infty$ predicen un efecto finito en el umbral efectivo debido a la adición de una segunda fase, independientemente del contraste en los umbrales locales. En dicho límite de no linealidad, las estimaciones GSEC para sistemas materiales cuya fase $r=2$ es un conductor iónico ideal $\left(G_{0}^{(2)}=0\right)$ pueden ser reducidas a

$$
\frac{\widetilde{G}_{0}}{G_{0}^{(1)}}=\left(1-\theta^{(2)}\right)\left[\left(1+\sqrt{\frac{\theta^{(2)}}{2} \frac{g_{\|}(\widetilde{k})}{g(\widetilde{k})}}\right)^{2}+\frac{\theta^{(2)}}{2} \frac{g_{\perp}(\widetilde{k})}{g(\widetilde{k})}\right]^{-1 / 2}
$$

donde las funciones $g$ son

$$
\begin{aligned}
g_{\|}(\widetilde{k}) & =-\left(1-2 \theta^{(2)}+8 \theta^{(2)} \widetilde{k}\right)+\left(1+10 \theta^{(2)}+8 \theta^{(2)} \widetilde{k}\right) \widetilde{k} \alpha(\widetilde{k}) \\
& +\left(2-8 \theta^{(2)}+\left(1-10 \theta^{(2)}\right) \widetilde{k}\right) \widetilde{k} \alpha(\widetilde{k})^{2} \\
& -\left(4-8 \theta^{(2)}+\left(1+2 \theta^{(2)}\right) \widetilde{k}\right) \widetilde{k}^{2} \alpha(\widetilde{k})^{3}+2 \widetilde{k}^{3} \alpha(\widetilde{k})^{4} \\
g_{\perp}(\widetilde{k}) & =\left(1-\theta^{(2)}\right) \widetilde{k}(\widetilde{k} \alpha(\widetilde{k})-1)^{2}(3-(2+\widetilde{k}) \alpha(\widetilde{k})) \\
g(\widetilde{k}) & =\frac{\theta^{(2)}}{2}\left(1-2 \theta^{(2)}\right)-\left(1-3 \theta^{(2)}-2\left(\theta^{(2)}\right)^{2}\right) \widetilde{k}+\left(1-\theta^{(2)}\right) \theta^{(2)} \widetilde{k}^{2} \\
& +\left[\left(2-\frac{13}{2} \theta^{(2)}-\left(\theta^{(2)}\right)^{2}\right) \widetilde{k}+2\left(1-4 \theta^{(2)}+\left(\theta^{(2)}\right)^{2}\right) \widetilde{k}^{2}+\theta^{(2)}\left(1-\theta^{(2)}\right) \widetilde{k}^{3}\right] \alpha(\widetilde{k}) \\
& -\left[\left(1-2 \theta^{(2)}-2\left(\theta^{(2)}\right)^{2}\right)+\left(4-\frac{25}{2} \theta^{(2)}+4\left(\theta^{(2)}\right)^{2}\right) \widetilde{k}+\left(1+\theta^{(2)}-2\left(\theta^{(2)}\right)^{2}\right) \widetilde{k} \widetilde{k}^{2}\right] \widetilde{k} \alpha(\widetilde{k})^{2} \\
& +\left[2\left(1-2 \theta^{(2)}\right)+\left(2-\frac{\theta^{(2)}}{2}\right) \widetilde{k}\right] \widetilde{k}^{2} \alpha(\widetilde{k})^{3}-\widetilde{k}^{3} \alpha(\widetilde{k})^{4} .
\end{aligned}
$$

Dichas funciones son funciones de la relación efectiva de anisotropía $\widetilde{k}=\widetilde{M}_{\|} / \widetilde{M}_{\perp}$, que es solución de la ecuación no lineal

$$
\frac{\theta^{(2)}}{2} \frac{g_{\|}(\widetilde{k})}{g(\widetilde{k})}=\frac{1}{(k-1)^{2}}
$$


con la relación de anisotropía $k$ de la fase $r=1$ dada por

$$
k=\frac{1-\widetilde{k} \alpha(\widetilde{k})}{1-\alpha(\widetilde{k})} \frac{\theta^{(2)}+\widetilde{k}\left(1-\theta^{(2)}\right)-\widetilde{k} \alpha(\widetilde{k})}{1-2 \theta^{(2)}(1-\widetilde{k})-\widetilde{k} \alpha(\widetilde{k})}
$$

Por otro lado, las estimaciones GSEC ${ }^{* 1}$ para materiales cuya fase $r=2$ es un aislante iónico ideal $\left(G_{0}^{(2)}=\infty\right)$ pueden reducirse a

$$
\frac{\widetilde{G}_{0}}{G_{0}^{(1)}}=\left[\left(1+\sqrt{\theta^{(2)} \frac{h_{\|}(\widetilde{k})}{h(\widetilde{k})}}\right)^{2}+\theta^{(2)} \frac{h_{\perp}(\widetilde{k})}{h(\widetilde{k})}\right]^{1 / 2}-\sqrt{\theta^{(2)} \frac{h_{\|}(\widetilde{k})}{h(\widetilde{k})}}
$$

donde las funciones $h$ son

$$
\begin{aligned}
h_{\|}(\widetilde{k}) & =\left[(1+\widetilde{k}(\alpha(\widetilde{k})-2))^{2}(\widetilde{k} \alpha(\widetilde{k})(2 \alpha(\widetilde{k})-1)-1)\right. \\
& +2 \theta^{(2)}\left(1-\widetilde{k}\left(6-7 \alpha(\widetilde{k})+4 \alpha(\widetilde{k})^{2}\right)+\widetilde{k}^{2}\left(2+\alpha(\widetilde{k})^{2}\right)\right. \\
& \left.\left.+\widetilde{k}^{3} \alpha(\widetilde{k})\left(2-6 \alpha(\widetilde{k})+3 \alpha(\widetilde{k})^{2}\right)\right)\right] \widetilde{k} \\
h_{\perp}(\widetilde{k}) & =\left(1-\theta^{(2)}\right)\left[1-\widetilde{k}(2-\alpha(\widetilde{k})]^{2}[3-(2+\widetilde{k}) \alpha(\widetilde{k})]\right. \\
h(\widetilde{k}) & =-2\left(-1+2 \widetilde{k}+(\alpha(\widetilde{k})-2) \alpha(\widetilde{k}) \widetilde{k}^{2}\right)^{2} \\
& +4\left(\theta^{(2)}\right)^{2}(\widetilde{k}-1)^{2}\left(-1+(\alpha(\widetilde{k})-1) \widetilde{k}^{2} \alpha(\widetilde{k})\right. \\
& +\widetilde{k}(2 \alpha(\widetilde{k})-1))+\theta^{(2)}(6-\widetilde{k}(13+8 \alpha(\widetilde{k})) \\
& +\widetilde{k}{ }^{4} \alpha(\widetilde{k})\left(4+4 \alpha(\widetilde{k})-5 \alpha(\widetilde{k})^{2}\right)+\widetilde{k}^{2}(12+17 \alpha(\widetilde{k}) \\
& \left.\left.-2 \alpha(\widetilde{k})^{2}\right)+\widetilde{k}^{3}\left(4-40 \alpha(\widetilde{k})+25 \alpha(\widetilde{k})^{2}-4 \alpha(\widetilde{k})^{3}\right)\right) .
\end{aligned}
$$

Éstas son funciones de la relación de anisotropía efectiva $\widetilde{k}$, que son solución de la ecuación no lineal

$$
\left(1+{\sqrt{\theta^{(2)} \frac{h_{\|}(\widetilde{k})}{h(\widetilde{k})}}}^{2}+\theta^{(2)} \frac{h_{\perp}(\widetilde{k})}{h(\widetilde{k})}=\left(1+\frac{k-1}{k} \sqrt{\theta^{(2)} \frac{h_{\|}(\widetilde{k})}{h(\widetilde{k})}}\right)^{2}\right.
$$

\footnotetext{
${ }^{1}$ En este caso, las expresiones matemáticas obtenidas a través de la formulación dual son más simples que las obtenidas con la formulación primaria.
} 
con la relación de anisotropías $k$ de la fase $r=1$ dada por

$$
k=(\widetilde{k} \alpha(\widetilde{k})-1) \widetilde{k} \frac{1+\widetilde{k}(\alpha(\widetilde{k})-2)+2 \theta^{(2)}(\widetilde{k}-1)}{\left[(\widetilde{k} \alpha(\widetilde{k})-1)-\theta^{(2)}(\widetilde{k}-1)\right][1+(\alpha(\widetilde{k})-2) \widetilde{k}]}
$$

Dichas estimaciones son graficadas en las Figuras 7.2-c y -d en función de la fracción volumétrica $\theta^{(2)}$. En el límite diluido $\theta^{(2)} \rightarrow 0$ dichas estimaciones coinciden a primer orden con las expansiones diluidas de las estimaciones correspondientes del tipo MGA. Esto se debe a que la estimaciones EMA y MGA en las cuales están basadas presentan el mismo comportamiento en concentraciones diluidas. De esta manera, las estimaciones GSEC basadas en las aproximaciones EMA también presentan singularidades para concentraciones diluidas y son consistentes con las localizaciones de los campos, como fue discutido en el contexto de las aproximaciones MGA. Sin embargo, para altas concentraciones $\theta^{(2)}$ las estimaciones GSEC del tipo EMA presentan un comportamiento percolativo acercándose a una fracción volumétrica crítica $\theta_{c}^{(2)}<1$. Dicha percolación es la misma que la presente en las aproximaciones EMA lineales en las cuales las estimaciones GESC están basadas, es decir dependientes de la dimensión espacial de los campos pero no de la no linealidad del material, como es esperable. En el caso que la fase $r=2$ sea un aislante iónico ideal $\left(G_{0}^{(2)}=\infty\right)$ la percolación ocurre en el valor crítico $\theta_{c}^{(2)}=2 / 3$, mientras que en el caso opuesto en que la fase $r=2$ sea un conductor ideal $\left(G_{0}^{(2)}=0\right)$ la percolación aparece en un valor crítico menor $\theta_{c}^{(2)}=1 / 3$. Cerca de dichos límites de percolación la resistividad efectiva no lineal se comporta como $\widetilde{G}_{0} \sim G_{0}^{(1)}\left(\theta_{c}^{(2)}-\theta^{(2)}\right)^{ \pm s}$, donde $s$ es un exponente crítico. Dado que las estimaciones SEC son cotas en sí mismas, que también presentan percolación, dicho exponente debe satisfacer el límite inferior $s \geq 1 / 2$ para $G_{0}^{(2)}=\infty$ y el límite superior $s \leq 1 / 2$ para $G_{0}^{(2)}=0$. Expandiendo las expresiones (7.14) y (7.20) puede verificarse que las estimaciones GSEC presentan un comportamiento percolativo con exponentes $s= \pm 1 / 2$, que son idénticos a los correspondientes a los límites. En esta observación, vale la pena destacar que una técnica lineal de comparación más antigua propuesta por Ponte Castañeda \& Kailasam (1997) y basada en una linealización tangente alrededor de los promedios de las fases llevaba a exponentes críticos de $s=0$ para $G_{0}^{(2)}=\infty$ y $s=1$ para $G_{0}^{(2)}=0$, violando por ende las cotas. Como ya fue indicado por Ponte Castañeda (2001), esta mejora que presentan las 
estimaciones de la secante generalizada en comparación con las estimaciones tangentes, es directamente atribuible al uso de la fluctuaciones de los campos — que quedan no acotadas en la percolación - en el esquema de linealización.

Se concluye esta discusión remarcando que el sólido lineal de comparación en el cual se apoyan las estimaciones GSEC basadas en una aproximación del tipo EMA pueden presentar tensores de movilidad negativos definidos cuando la no linealidad es lo suficientemente alta. De hecho, aun sea $\widetilde{k}>0$ para cualquier valor de $m$, la relación de anisotropía asociada $k$ pasa a ser negativa para valores de $m$ suficientemente altos. Rigurosamente, el potencial efectivo correspondiente $\widetilde{U}_{0}$, como es detallado en (6.10), deja de ser un mínimo y se convierte en un valor estacionario. Este hecho genera algunas dudas sobre la validez de dichas estimaciones EMA para compuestos cuyas fases tienen comportamientos del tipo ley de la potencia con no linealidades suficientemente altas. Estas cuestiones no son exclusivamente debidas al uso de estimaciones lineales EMA, ya Idiart \& Ponte Castañeda (2005) identificaron esta situación en estimaciones de la secante generalizada para compuestos viscoplásticos utilizando un método lineal diferente. Sin embargo, las estimaciones para la resistividad alineal efectiva siguen satisfaciendo las cotas rigurosas y arrojan predicciones razonables. Durante el curso del presente trabajo de tesis, Ponte Castañeda (2015) propuso una formulación del método de la secante generalizada que podría dar predicciones más precisas sin presentar esta falencia matemática. Si bien dicha técnica está solamente disponible hoy en día para sólidos cristalinos, podría ser adaptada para otro tipo de sólidos incluyendo los considerados aquí. Por ende más esfuerzos deberían ser dedicados al estudio de dicho tema. 


\section{Capítulo 8}

\section{Conclusiones}

Se ha propuesto un formalismo teórico de medios continuos multiescala para describir el transporte iónico a través de electrolitos sólidos microestructurados. Las ecuaciones de campo para cada escala se obtuvieron mediante el método de convergencia multiescala. El formalismo es válido para electrolitos con un número arbitrario de fases microestructurales capaces de polarizarse eléctricamente y de transportar un número arbitrario de especies iónicas por electromigración y difusión. La respuesta de cada fase se describe mediante su energía libre y un potencial de disipación convexos en conformidad con el formalismo de materiales estándar generalizados. Esto garantiza la consistencia termodinámica de las predicciones resultantes. El formalismo permite pues describir respuestas tanto lineales como no lineales. En el límite de homogenización, las ecuaciones de campo microscópicas se desacoplan de manera tal que la difusión de cada especie iónica y la polarización eléctrica del medio pueden tratarse separadamente. Las capacidades del formalismo fueron evaluadas tanto en el contexto de microgeometrías resolubles como en el contexto de microestructuras estocásticas.

La formulación multiescala desarrollada fue aplicada, a modo de casos de estudio, a diferentes microgeometrías en particular. En primer lugar se analizó un sistema cilindrítico lineal en el cual se pudo concluir que la presencia de una mayor cristalinidad no implica necesariamente una menor movilidad macroscópica, contrariamente a lo usualmente divulgado en la bibliografía de referencia. En la misma línea se pudo concluir que la fase amorfa no 
presenta siempre mejores propiedades de trasporte que la fase cristalina.

También fueron estudiados sistemas materiales no lineales del tipo matriz-partícula y granulares, utilizando una lineaización basada en el método de la secante generalizada. Los resultados obtenidos en dichos casos son consistentes con las cotas estrictas del problema, incluso para fuertes no linealidades y concentraciones entorno a la percolación. Dentro de los resultados obtenidos además se pudo verificar que para los casos de respuestas del tipo umbral los resultados son consistentes con flujos iónicos localizados en caminos de mínima energía.

Más allá de los resultados particulares obtenidos a través de este desarrollo, si bien dicho formalismo permitirá estudiar numerosos problemas de interés para el desarrollo de electrolitos sólidos, resta considerar varias cuestiones importantes que no fueron abordadas en el presente trabajo. En primer lugar, resta considerar el problema multiescala no estacionario. Recientemente, Salvadori et al. (2014) y Brassart \& Stanier (2016) consideraron problemas de difusión multiescala no estacionarios valiéndose del principio de Hill-Mandel en lugar del método de convergencia multiescala aquí utilizado. En estos trabajos se encuentra que el flujo de iones macroscópico está dado no sólo por el promedio volumétrico del flujo de iones microscópico en el elemento de volumen representativo sino que también depende de la tasa de variación de la concentración de iones en dicho volumen. En el caso de flujos estacionarios esta última contribución se anula y la relación entre los flujos microscópicos y macroscópicos coincide con la obtenida en el presente trabajo. Pero no es evidente qué relación surgirá para el problema no estacionario al utilizar el método de convergencia multiescala.

En segundo lugar, resta incorporar al formalismo efectos de interfaces materiales. Evidencias experimentales recientes (véase por ejemplo Cheng et al., 2015) plantean la posibilidad de que sean las capas amorfas encerradas entre zonas semicristalinas las de mayor conductividad que el resto de la microestructura semicristalina, siendo así dichas interfaces las que mayor peso tengan en la determinación de la conductividad iónica macroscópica del material. Además de dichas evidencias, se cree importante la posibilidad de considerar la presencia de doble capas eléctricas. Es probable que para ello deba incorporarse una densidad volumétrica de cuadripolos como descriptor adicional del estado material. Ello conllevará a teorías constitutivas de tipo gradiente con condiciones de salto en las interfaces materiales. Si bien 
este tipo de formalismos no han podido ser utilizados aún en el contexto de microestructuras estocásticas, ciertamente podrían utilizarse para generar predicciones basadas en microgeometrías resolubles como las consideradas en el Capítulo 4 (véase, por ejemplo, Idiart \& Fleck 2008).

Se ha mencionado ya que la deformación mecánica puede producir cambios significativos en la conductividad iónica de electrolitos poliméricos. En vista del fuerte desarrollo de la flexoelectrónica actualmente en curso, sería deseable pues desarrollar un formalismo teórico para el transporte iónico que tenga en cuenta las deformaciones del medio y la evolución de la microestructura concomitante. Teorías multiescala puramente mecánicas para materiales poliméricos microestructurados bajo grandes deformaciones ya han sido propuestas por Lopez-Pamies et al. (2008) y Racherla et al. (2010), entre otros. Por otro lado, teorías electromecánicas para sistemas bajo grandes deformaciones pero sin microestructura ya han sido propuestas por Xiao \& Bhattacharya (2008) y Bower et al. (2011), entre otros. Un formalismo para el transporte iónico a través de medios microestructurados deformables podría derivarse combinando elementos propuestos en estos trabajos.

En este punto creemos oportuno agregar que las caracterizaciones experimentales requeridas para apoyar las teorías a desarrollarse e incluso para las ya desarrolladas, tienen aún sectores vitales inexplorados en profundidad o incluso completamente inexplorados. En el presente trabajo se han citado varios trabajos e incluso se ha presentado un pequeño grupo de experimentos propios realizados sobre un caso modelo para probar la potencialidad de las teorías derivadas. Pero encontramos a lo largo de este trabajo de tesis una falta marcada de evidencia experimental detallada que permita disponer valores de los numerosos parámetros materiales necesarios para obtener resultados teóricos comparables con los experimentales. Creemos que algo similar ocurre con los incipientes desarrollos de teorías que combinen el transporte iónico con el comportamiento mecánicos de estos sólidos microestructurados.

Por último, se han identificado falencias en el método de linearización de tipo secante generalizada. Es posible que dichas falencias provengan del hecho de que el procedimiento variacional utilizado no es completamente estacionario respecto a las propiedades del medio lineal de comparación. Este último está caracterizado por tensores de movilidad y gradientes de referencia. La linearización tangente inicialmente propuesta por Ponte Castañeda (1996) 
se obtiene a partir de una condición de estacionaridad de la función objetivo respecto a los gradientes de referencia; los tensores de movilidad se identifican con la movilidad tangente de manera ad-hoc. Por otro lado, la linearización secante generalizada posteriormente propuesta por Ponte Castañeda (2001) y utilizada en el presente trabajo se obtiene a partir de una condición de estacionaridad de la función objetivo respecto a los tensores de movilidad; en este caso son los gradientes de referencia los que se identifican con los promedios por fase de manera ad-hoc. Hasta ahora no se había logrado derivar una linearización que surgiera de condiciones de estacionaridad respecto a ambas propiedades simultáneamente. Esto habría sido finalmente logrado en un trabajo publicado por Ponte Castañeda (2015) durante la redacción de estas líneas. Si esta nueva linearización completamente estacionaria resuelve en efecto las falencias encontradas, contaremos finalmente con un poderoso método de linearización para derivar teorías constitutivas no lineales de medios continuos microestructurados. 


\section{Apéndice}

A continuación se presentan las gráficas resultado de los experimentos que han permitido obtener los valores expuestos en el Capítulo 2. 


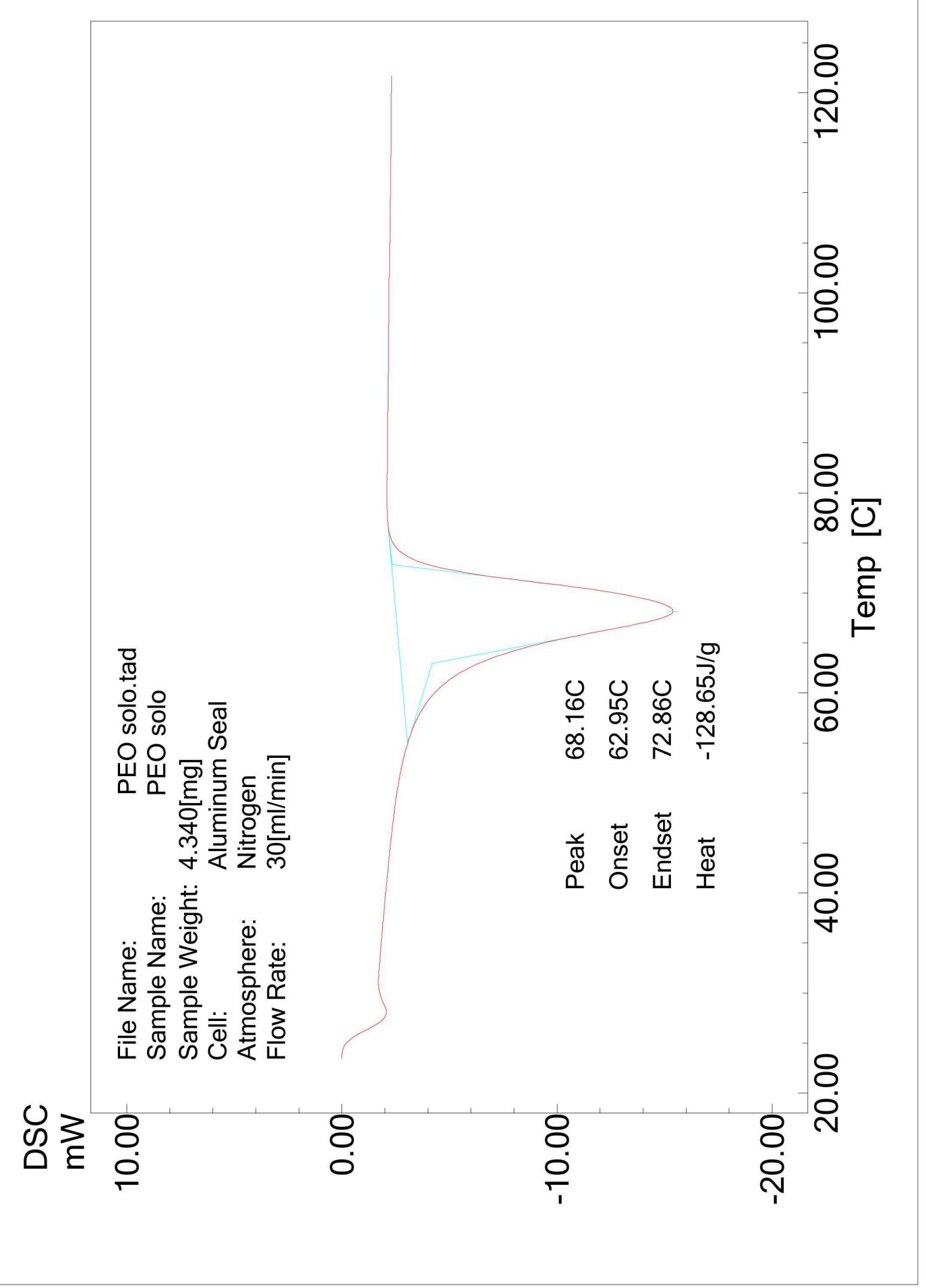

Figura A1: Curva resultado del análisis de calorimetría diferencial para probeta de PEO sin dopar 


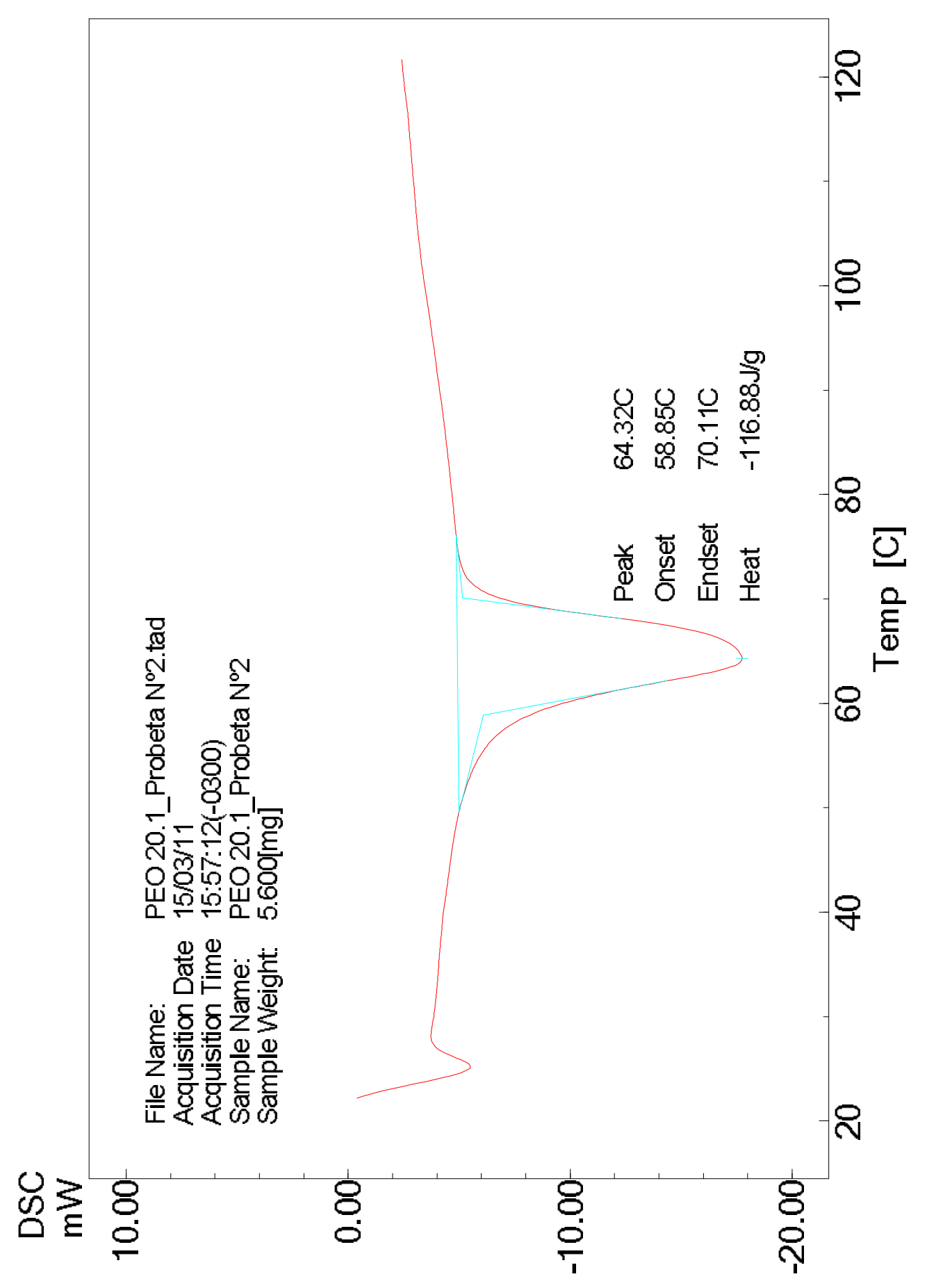

Figura A2: Curva resultado del análisis de calorimetría diferencial de la Probeta 2 de PEO:Li 20:1 - Día 1 


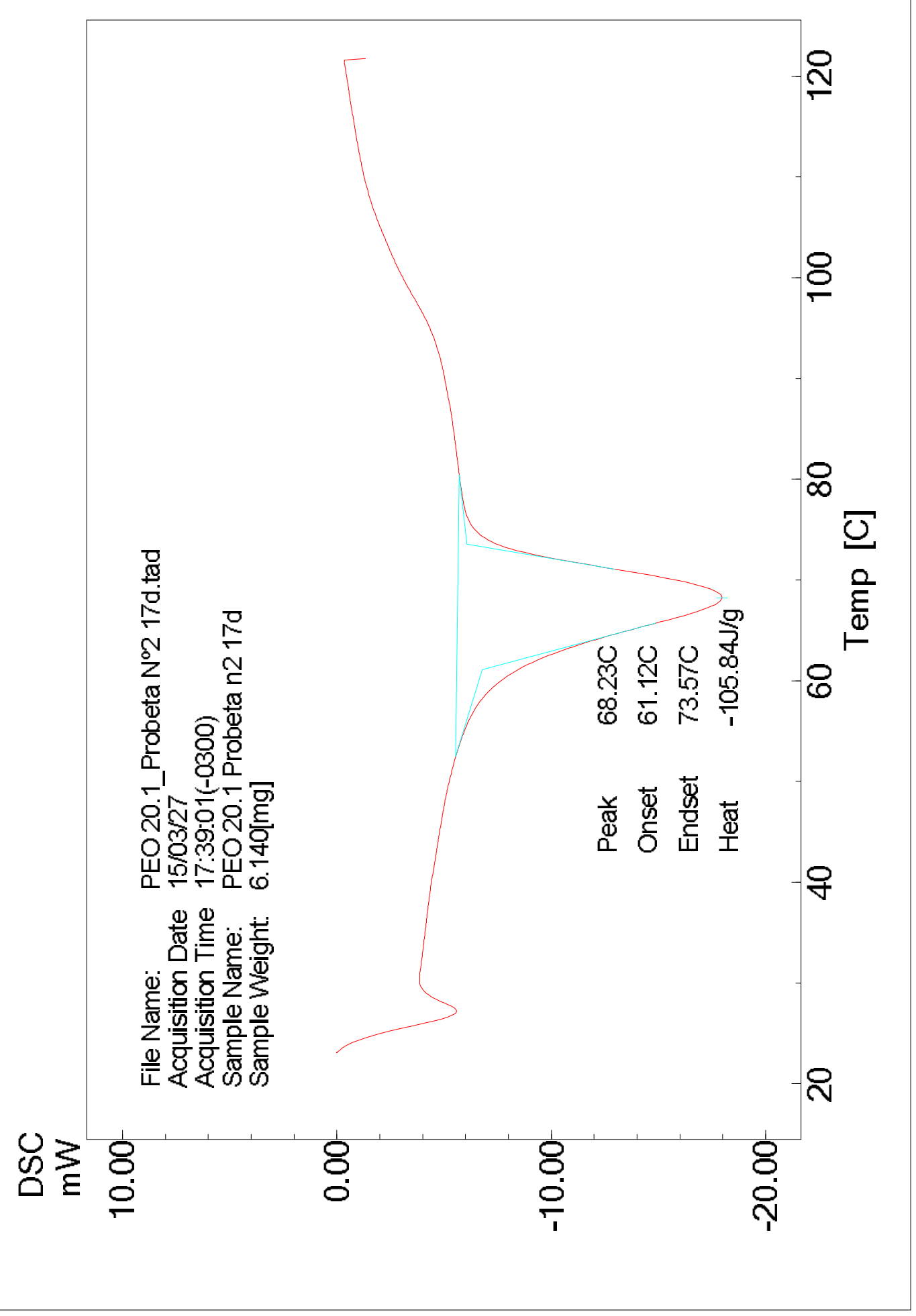

Figura A3: Curva resultado del análisis de calorimetría diferencial de la Probeta 2 de PEO:Li 20:1 - Día 17 


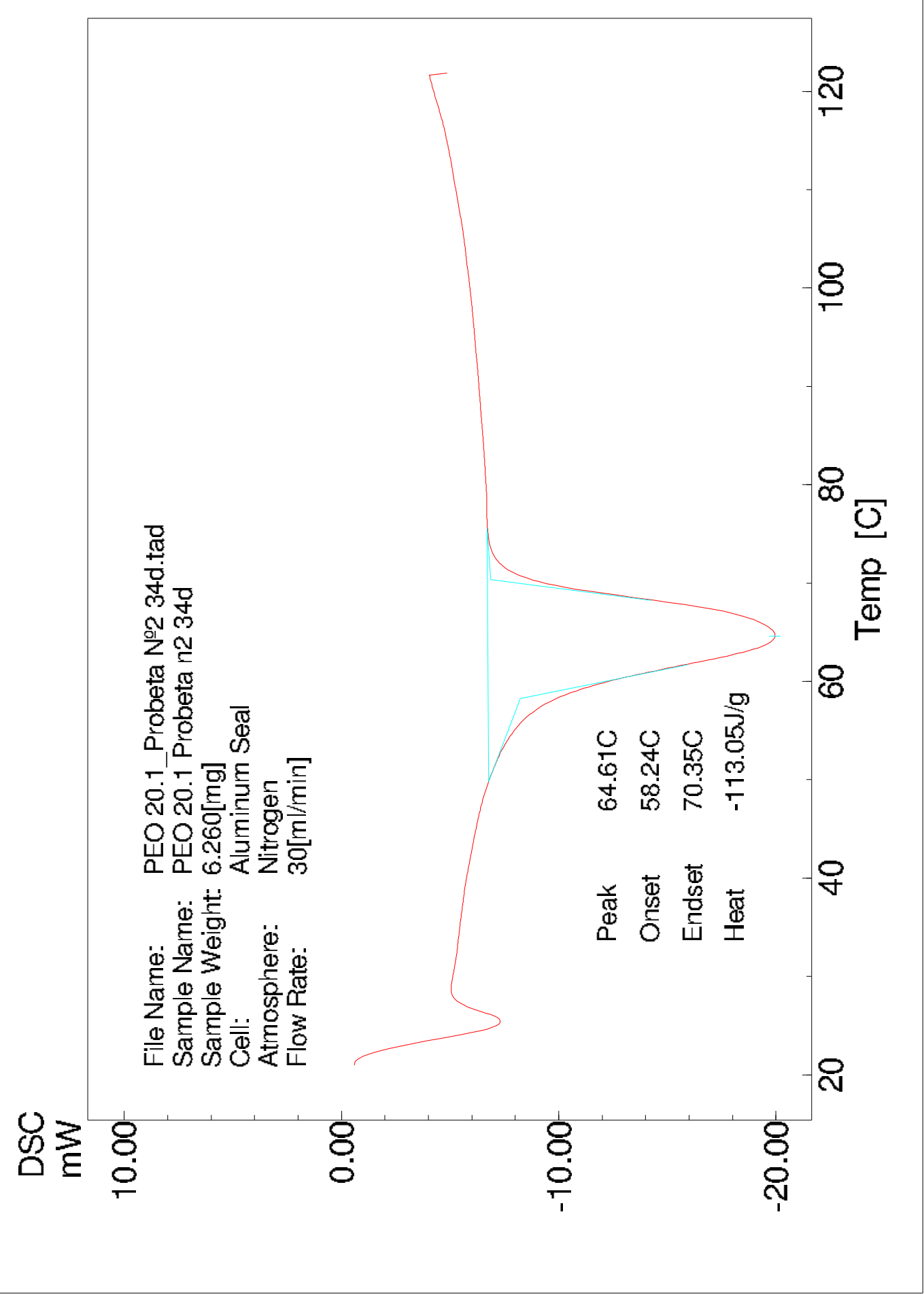

Figura A4: Curva resultado del análisis de calorimetría diferencial de la Probeta 2 de PEO:Li 20:1 - Día 34 


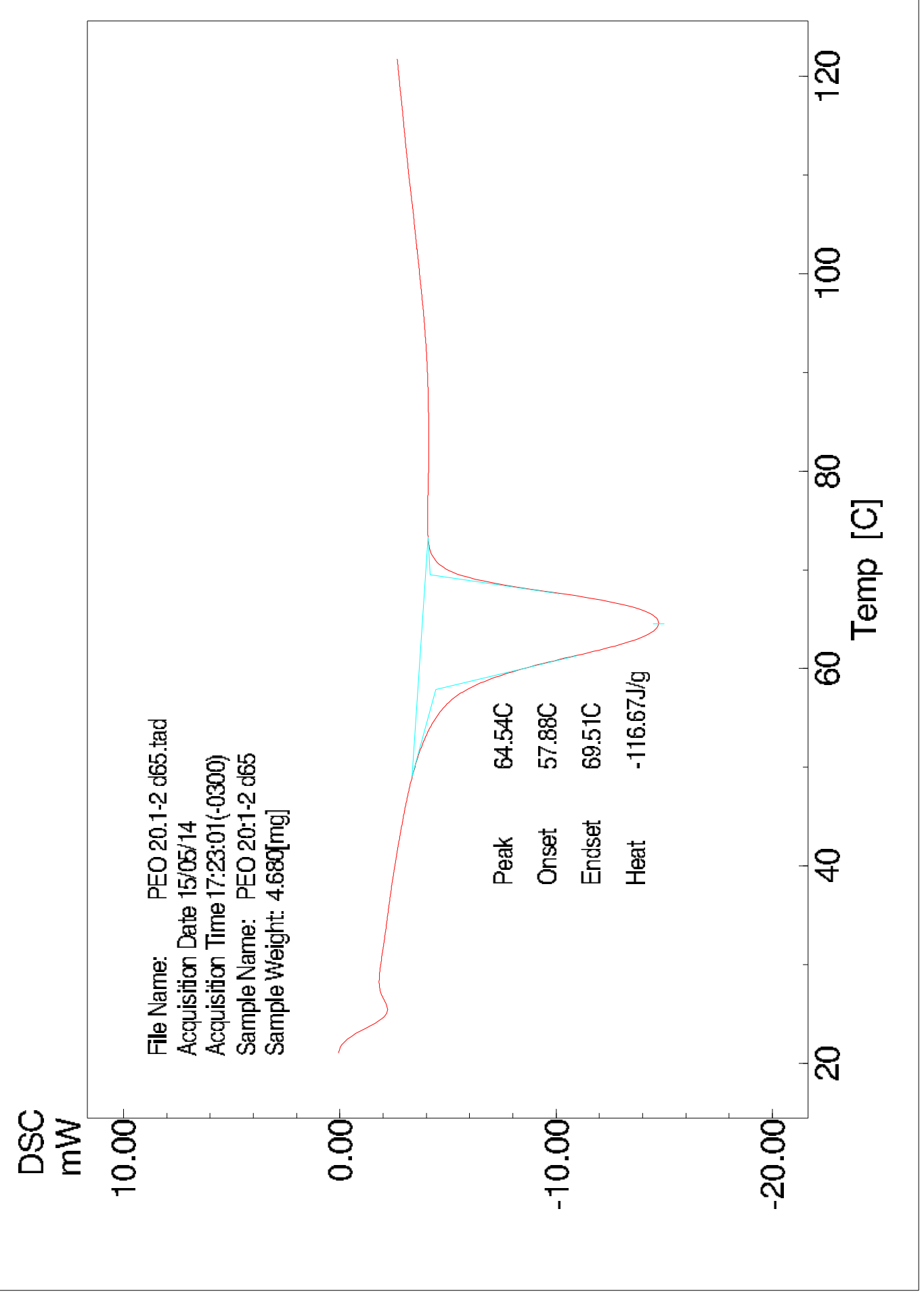

Figura A5: Curva resultado del análisis de calorimetría diferencial de la Probeta 2 de PEO:Li 20:1 - Día 65 


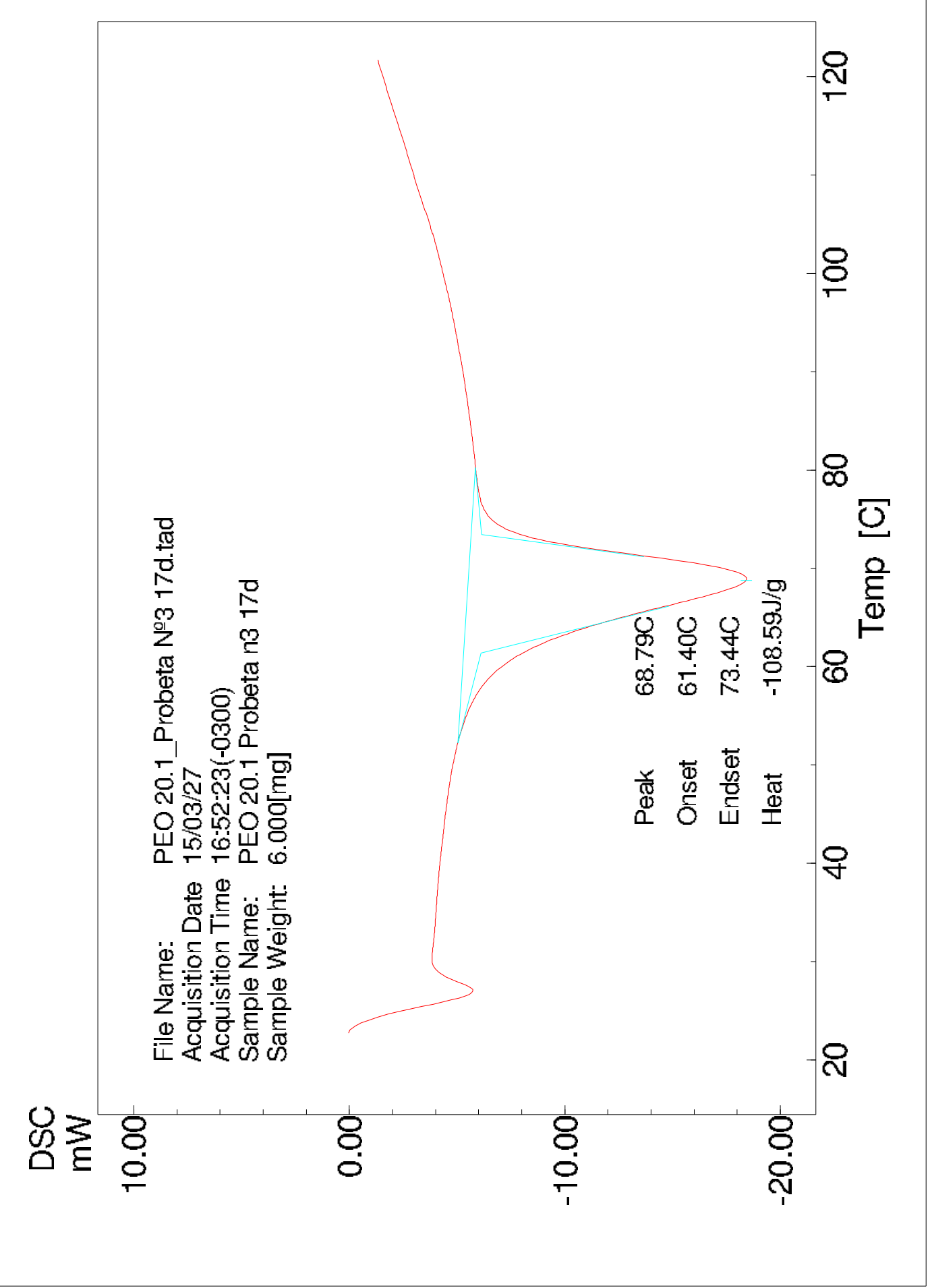

Figura A6: Curva resultado del análisis de calorimetría diferencial de la Probeta 3 de PEO:Li 20:1 - Día 17 


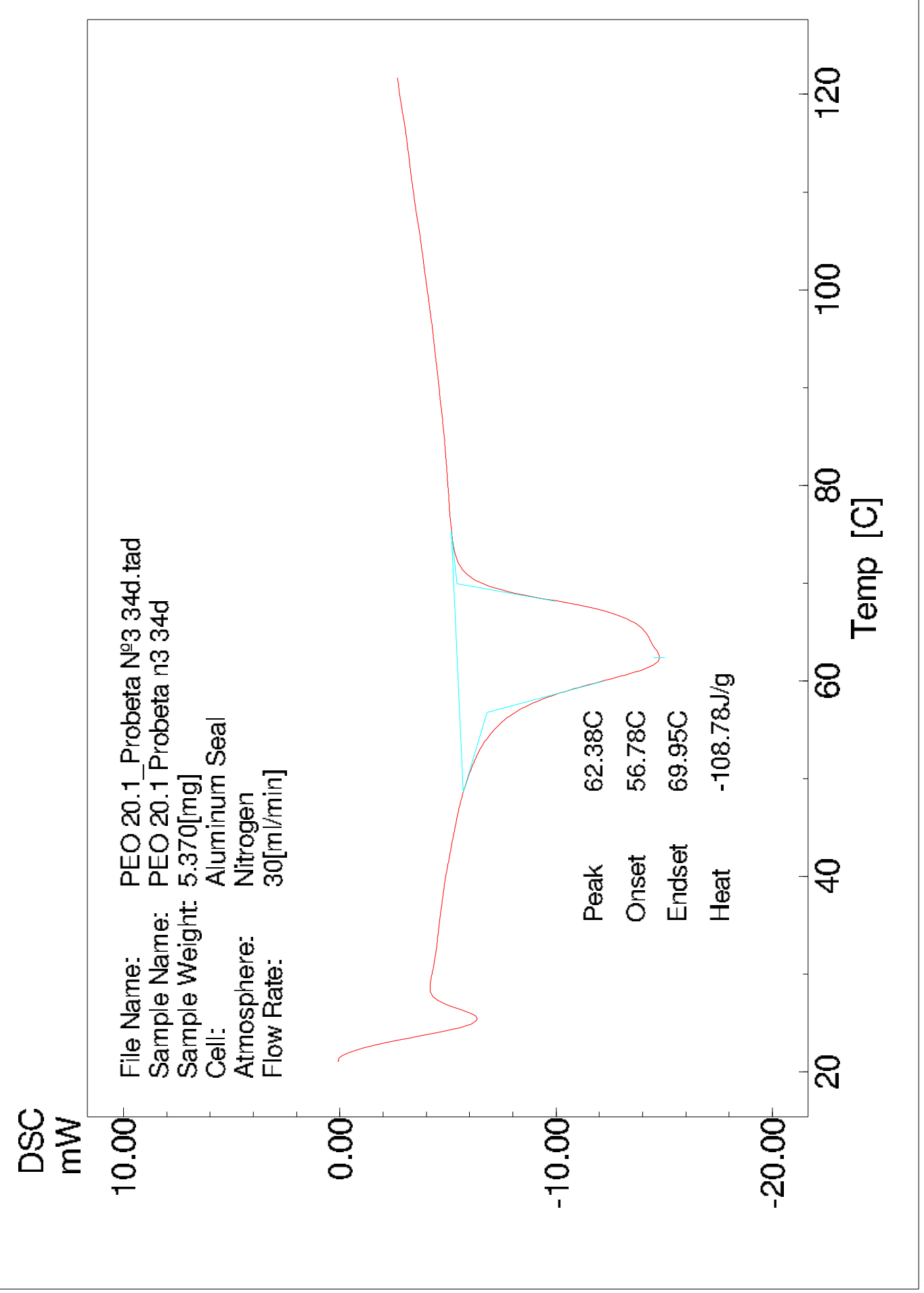

Figura A7: Curva resultado del análisis de calorimetría diferencial de la Probeta 3 de PEO:Li 20:1 - Día 34 


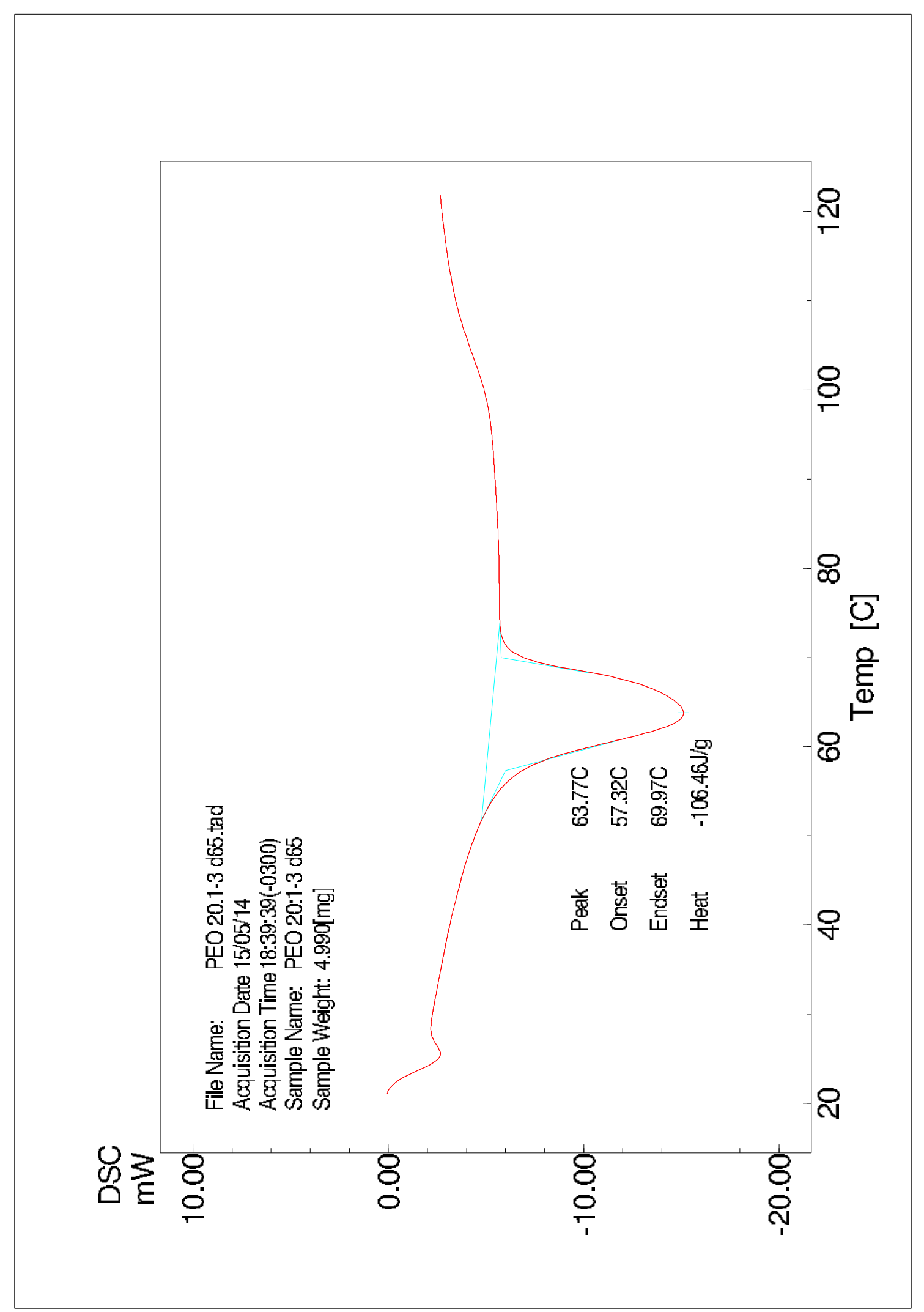

Figura A8: Curva resultado del análisis de calorimetría diferencial de la Probeta 3 de PEO:Li 20:1 - Día 65 
20:1

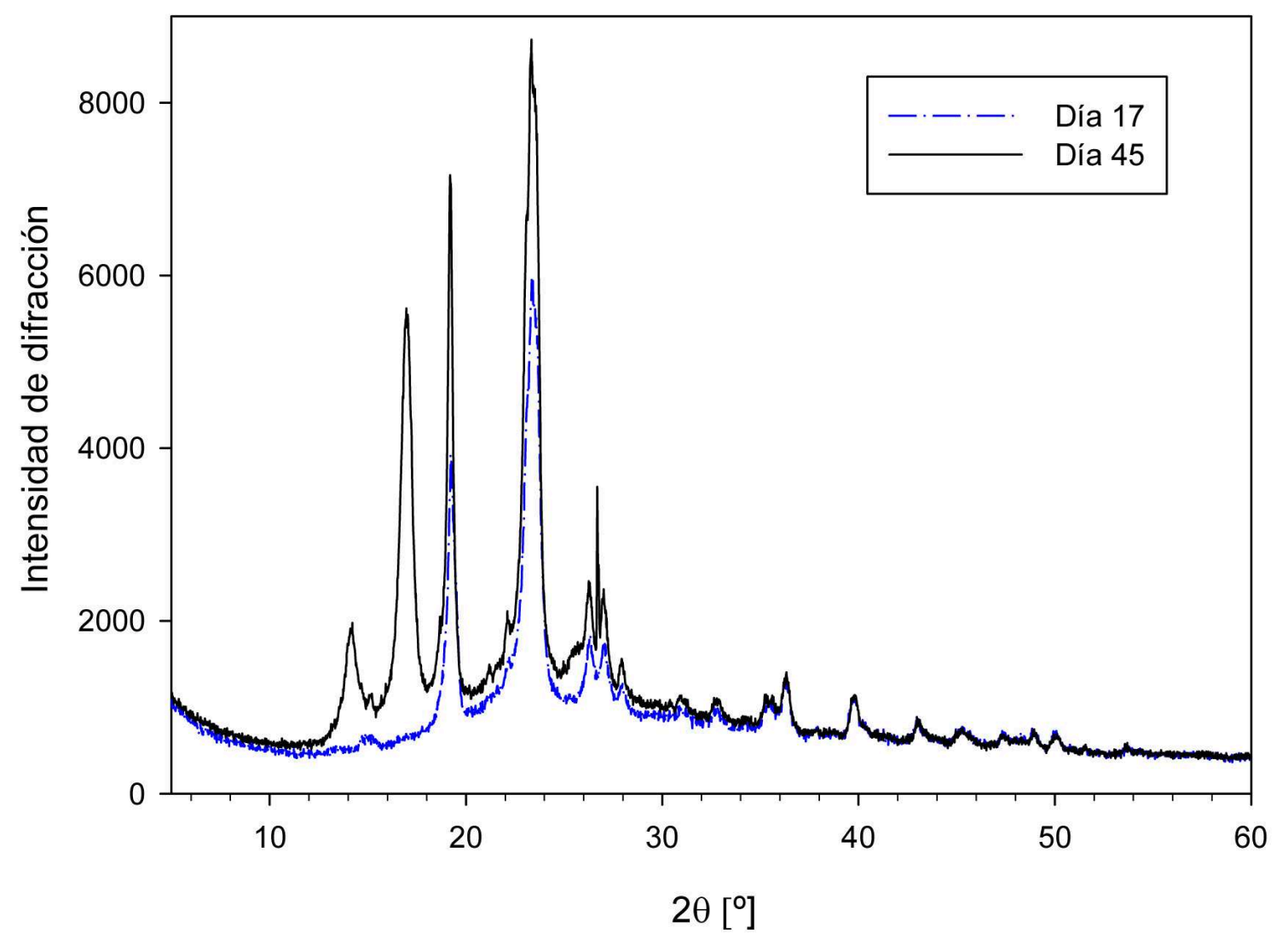

Figura A9: Curva resultado de los análisis de difracción de rayos X de probeta de PEO:Li 20:1 Días 17 y 45 


\section{Referencias}

- M. Agoras, P. Ponte Castañeda, (2011): Homogenization estimates for multi-scale nonlinear composites. Eur. J. Mech. A/Solids 30, 828-843.

- G. Allaire, M. Briane (1996): Multiscale convergence and reiterated homogenization. Proc. R. Soc. Edin. 126A, 297-342.

- G. Allaire, A. Mikelić, A. Piatnitski (2010): Homogenization of the linearized ionic transport equations in rigid periodic porous media. Journal of Mathematical Physics 51, 123103.

- L.E. Asp, S. Leijonmarck, T. Carlson, G. Lindbergh (2015): Realisation of structural battery composite materials. 20th International Conference on Composite Materials, Copenhagen.

- S.P.S. Badwal, J. Drennan (1994): Interfaces in zirconia based electrochemical systems and their influence on electrical properties. Science of Ceramic Interfaces II, Ed. J. Nowotny, Elsevier, Holanda.

- K.E. Barrett, D.R.S. Talbot (1996): Bounds for the effective properties of a nonlinear twophase composite dielectric. Proc. 8th Int. Symp. Continuum Models and Discrete Systems, pp. 92-99. World Scientific, Singapore.

- I.V. Belova, G.E. Murch (2005): Calculation of the effective conductivity and diffusivity in composite solid electrolytes. Journal of Physics and Chemistry of Solids 66, 722-728.

- C. Berthier, W. Gorecki, M. Minier, M.B. Armand, J.M. Chabagno, P. Rigaud (1983): Microscopic investigation of ionic conductivity in alkali metal salts-poly(ethylene oxide) adducts. Solid State Ionics 11, 91-95.

- R. Blumenfeld, D.J. Bergman (1991): Strongly nonlinear composite dielectrics: A perturbation method for finding the potential field and bulk effective properties. Phys. Rev. B 44, $7378-7386$. 
- T. Blythe, D. Bloor (2005): Electrical Properties of Polymers. Cambridge University Press, Reino Unido.

- A. Boudet (2003): Voyage au Coeur de la Matière Plastique. CNRS Editions, Francia.

- K. Bourbatache, O. Millet, A. Aït-Mohtar, O. Amiri, (2013): Chloride transfer in cementbased materials. Part 1. Theoretical basis and modelling. Int. J. Numer. Anal. Meth. Geomech. 37, 1614-1627.

- A.F. Bower, P.R. Guduru, V.A. Sethuraman (2011): A Finite Strain Model of Stress, Diffusion, Plastic Flow and Electrochemical Reactions in a Lithium-ion Half-cell. Journal of the Mechanics and Physics of Solids 59, 804-828.

- L. Brassart, L. Stanier (2016): Homogenization-based constitutive modelling for diffusion problems. 24th International Congress of Theoretical and Applied Mechanics, Montreal, Canadá.

- D.A.G. Bruggeman (1935): Berechnung verschiedener physikalischer Konstanten von heterogenen Substanzen Ann Phys. Leipzig 24, 636.

- F. Capuano, F. Croce, B. Scrosati (1991): Composite polymer electrolytes. Journal of Electrochemical Society 138, 1918-1922.

- J. Casado-Díaz, I. Gayte (2002): The two-scale convergence method applied to generalized Besicovitch spaces. Proc. R. Soc. Lond. A 458, 2925-2946.

- S. Cheng, D. Smith, Q. Pan, S. Wang and C. Li, (2015): Anisotropic ion transport in nanostructured solid polymer electrolytes. RSC Adv., en prensa.

- L. Christodoulou and J.D. Venables (2003): Multifunctional Materials Systems: The First Generation, JOM 55, 39-45.

- Ciocek, M., Sannier, L., Siekierski, M., Golodnitsky, D., Peled, E., Scrosati, B., Glowinkowski, S., Wieczorek, W., (2007): Ion transport phenomena in polymeric electrolytes. Electrochim. Acta 53, 1409-1416.

- R.D. Coleman, W. Noll (1963): The thermodynamics of elastic materials with heat conduction and viscosity. Arch. Ration. Mech. Anal. 13, 167-178.

- F. Croce, L. Persi, B. Scrosati, F. Serraino-Fiory, E. Plichta, M.A. Hendrickson (2001): Role of the ceramic fillers in enhancing the transport properties of composite polymer electrolytes. Electrochim. Acta 46, 2457-2461. 
- F. Croce, S.L. Sachetti, B. Scrosati (2006): Advanced, lithium batteries based on highperformance composite polymer electrolytes. J. Power Sources 162, 685-689.

- I.J. Curto Sillamoni, M. Idiart (2015): A model problem concerning ionic transport in microstructured solid electrolytes. Continuum Mechanics and Thermodynamics 27, 941-957.

- I.J. Curto Sillamoni, M.I. Idiart (2016): Nonlinear ionic transport through microstructured solid electrolytes: homogenization estimates. Modelling Simul. Mater. Sci. Eng. 24075008

- A. Donev, C.E. Musolff, P.M. Duxbury (2002): Random manifolds in non-linear resistor networks: applications to varistors and superconductors. J. Phys. A: Math. Gen. 35, L327.

- P.M. Duxbury, P.D. Beale, H. Bak, P.A. Shroeder (1990): J. Phys. D 23, 1546.

- P.M. Duxbury, P.D. Beale, C. Moukarzel (1995): Phys. Rev. B 51, 3476.

- P.M. Duxbury, E.S. McGarrity, E.A. Holm (2006): Critical manifolds in non-linear response of complex materials. Mech. Mater. 38, 757-771.

- J. Fergus (2010): Ceramic and polymeric solid electrolytes for lithium-ion batteries. Journal of Power Sources 195, 4554-4569.

- A. Heuer, S. Murugavel, B. Roling (2005): Nonlinear ionic conductivity of thin solid electrolyte samples: Comparison between theory and experiment. Physical Review B 72, 174304.

- H Fritzsche and M. Pollak. Hopping and Related Phenomena. World Scientific, Londres, Reino Unido, 1990.

- S.K. Fullerton-Shirey, J.K. Maranas (2009): Effect of $\mathrm{LiClO}_{4}$ on the structure and mobility of PEO-based solid polymer electrolytes. Macromolecules 42, 2142-2156.

- K. Funke (2013): Solid state ionics: from Michael Faraday to green energy-the European dimension. Sci. Technol. Adv. Mater. 14, 043502.

- P. Germain, Q.S. Nguyen, P. Suquet (1983): Continuum thermodynamics. J. App. Mech. 50, $1010-1020$.

- L. Gitelman, M. Israeli, A. Averbuch, M. Nathan, Z. Schuss, D. Golodnitsky (2008): Polymer geometry and $\mathrm{Li}^{+}$conduction in poly(ethylene oxide). J. Comp. Phys. 227, 8437-8447.

- L. Gitelman, M. Israeli, A. Averbuch, N. Nathan, Z. Schuss, D. Golodnitsky (2008): Modeling and simulation of Li-ion conduction in poly(ethylene oxide). Journal of Computational Physics 227, 1162-1175. 
- D. Golodnitsky, E. Livshits, A. Ulus, Z. Barkay, I. Lapides, E. Peled, S.H. Chung, S. Greenbaum (2001): Fast ion transport phenomena in oriented semicrystalline LiI-P(EO $)_{n}$-based polymer electrolytes. Journal of Physical Chemistry A 105, 10098-10106.

- D. Golodnitsky, E. Peled (2000): Stretching-induced conductivity enhancement of LiI-(PEO)polymer electrolyte. Electrochimica Acta 45, 1431-1436.

- J.D. Heumen, J.R. Stevens (1995): The role of lithium salts in the conductivity and phase morphology of a thermoplastic polyurethane. Macromolecules 28, 4268-4277.

- M.I. Idiart, P. Ponte Castañeda (2005): Second-order estimates for nonlinear isotropic composites with spherical pores and rigid particles. C. R. Mecanique 333, 147-154.

- M.I. Idiart, N.A. Fleck (2008): The effect of interfaces on the plastic behavior of periodic composites. Philosophical Magazine 88, 3633-3653.

- M.I. Idiart, F. Willot, Y-P Pellegrini, P. Ponte Castañeda (2009): Infinite-contrast periodic composites with strongly nonlinear behavior: Effective-medium theory versus full-field simulations. Int. J. Solids Struct. 46, 3365-3382.

- S. Kalnaus, A.S. Sabau, S. Newman, W.E. Tenhaeff, C. Daniel, N.J. Dudney (2011): Effective conductivity of particulate polymer composite electrolytes using random resistor network method. Solid State Ionics 199-200, 44-53.

- R.J. Latham, R.G. Linford (1987): Ionic and electronic transport. Electrochemical Science and Technology of Polymers 1, Ed. R.G. Linford. Springer.

- O. Lopez-Pamies, R. Garcia, E. Chabert, J.-Y. Cavaillé, P. Ponte Castañeda (2008): Multiscale modeling of oriented thermoplastic elastomers with lamellar morphology. Journal of the Mechanics and Physics of Solids 56, 3206-3223.

- M. Marzantowicz, F. Krok, J.R. Dygas, Z. Florjańczyk, E. Zygadlo-Monikowska, (2008): The influence of phase segregationon properties of semicrystalline PEO:LiTFSI electrolytes. Solid State Ionics 179 1670-1678.

- G.W. Milton (2002): The theory of composites. Cambridge University Press, Reino Unido.

- T. Minami, M. Tatsumisago, M. Wakihara, C. Iwakura, S. Kohjiya, I. Tanaka (2005): Solid state ionics for batteries. Springer, Alemania.

- P. Ponte Castañeda (1992): Bounds and estimates for the properties of nonlinear heterogeneous systems. Philosophical Transactions: Physical Sciences and Engineering 340, 531-567. 
- P. Ponte Castañeda (1996): Exact second-order estimates for the effective mechanical properties of nonlinear composite materials. Journal of the Mechanics and Physics of Solids 44, $827-862$.

- P. Ponte Castañeda, M. Kailasam (1997): Nonlinear electrical conductivity in heterogeneous media. Proceedings of the Royal Society of London A 453, 793-816.

- P. Ponte Castaeda (2001): Second-order theory for nonlinear dielectric composites incorporating field fluctuations. Physical Review B 64, 214205.

- P. Ponte Castañeda P (2015): Fully optimized second-order variational estimates for the macroscopic response and field statistics in viscoplastic crystalline composites. Proc. R. Soc. A, 47120150665 .

- V. Racherla, O. Lopez-Pamies, P. Ponte Castaeda (2010): Macroscopic response and stability in lamellar nanostructured elastomers with "oriented" and "unoriented" polydomain microstructures. Mechanics of Materials 42, 451-468.

- S.C. Roberts, G.S. Aglietti (2007): Satellite multi-functional power structure: feasibility and mass savings. Proc. IMechE, Part G: J. Aerospace Engineering 222, 41-51.

- C.D. Robitaille, D. Fauteux (1986): Phase diagrams and conductivity characterization of some PEO-LiX electrolytes. J. Electrochem. Soc. 133, 315-325.

- S. Roux, D. François (1991): A simple model for ductile fracture of porous materials. Scripta Met. 25, 1087-1092.

- A. Salvadori, E. Bosco, D. Grazioli (2014): A computational homogenization approach for Li-ion battery cells: Part 1 - formulation. Journal of the Mechanics and Physics of Solids 65, $114-137$.

- M. Schmuck, M.Z. Bazant (2015): Homogenization of the Poisson-Nernst-Planck equations for ion transport in charged porous media. SIAM Journal on Applied Mathematics 75, 13691401.

- K. Schulgasser (1983): Sphere assemblage model for polycrystals and symmetric materials. J. Appl. Phys. 54, 1380

- C.A.C. Sequeira, A. Hooper (1992): Electrical breakdown phenomena in (PEO)xLiF3CSO3 ion-conducting polymers. Journal of Power Sources 40, 271-282.

- C. Sequeira, D. Santos. Polymer Electrolytes: Fundamentals and Applications. Ed. Woodhead Publishing, 2010. 
- M. Siekierski, K. Nadara (2005): Modeling of conductivity in composites with random resistor networks. Electrochimica Acta 50, 3796-3804.

- M. Siekierski, W. Wieczorek, K. Nadara (2007): Mesoscale models of conductivity in polymeric electrolytes-A comparative study. Electrochim. Acta 53, 1556-1567.

- P. Suquet (1987): Elements of homogenization for inelastic solid mechanics. In: Homogenization techniques for composite media (E. Sanchez-Palencia and A. Zaoui, eds.). Lecture Notes in Physics 272. Springer-Verlag, Berlin, pp. 193-278.

- J. Syzdek, M. Armand, M. Gizowska, M. Marcinek, E. Sasim, M. Szafran, W. Wieczorek (2009): Ceramic-in-polymer versus polymer-in-ceramic polymeric electrolytes-A novel approach. Journal of Power Sources 194, 66-72.

- M. Tatsumisago, M. Nagao, A. Hayashi (2013): Recent development of sulfide solid electrolytes and interfacial modification for all-solid-state rechargeable lithium batteries. Journal of Asian Ceramic Societies 1, 17-25.

- V.L. Teofilo, J.N. Nadell (1998): Aerospace lithium solid polymer batteries. IEEE AES Systems Magazine 13, 33-36.

- J.R. Willis (1983): The overall elastic response of composite materials. Journal of Applied Mechanics 50, 1202-1209.

- Y. Xiao, K. Bhattacharya (2008): A continuum theory of deformable, semiconducting ferroelectrics. Arch. Rational Mech. Anal. 189, 59-95. 